Florida International University

FIU Digital Commons

\title{
Towards In-situ Based Printed Sensor Systems for Real-Time Soil- Root Nutrient Monitoring and Prediction with Polynomial Regression
}

Lamar K. Burton

Florida International University Department of Electrical and Computer Engineering, Iburt004@fiu.edu

Follow this and additional works at: https://digitalcommons.fiu.edu/etd

Part of the Electrical and Computer Engineering Commons

\section{Recommended Citation}

Burton, Lamar K., "Towards In-situ Based Printed Sensor Systems for Real-Time Soil-Root Nutrient Monitoring and Prediction with Polynomial Regression" (2020). FIU Electronic Theses and Dissertations. 4394.

https://digitalcommons.fiu.edu/etd/4394

This work is brought to you for free and open access by the University Graduate School at FIU Digital Commons. It has been accepted for inclusion in FIU Electronic Theses and Dissertations by an authorized administrator of FIU Digital Commons. For more information, please contact dcc@fiu.edu. 


\section{FLORIDA INTERNATIONAL UNIVERSITY}

Miami, Florida

TOWARDS IN-SITU BASED PRINTED SENSOR SYSTEMS FOR REAL TIME SOIL-ROOT NUTRIENT MONITORING AND PREDICTION WITH POLYNOMIAL REGRESSION

A dissertation submitted in partial fulfillment of the requirements for the degree of DOCTOR OF PHILOSOPHY

in

ELECTRICAL AND COMPUTER ENGINEERING

by

Lamar Burton 
To: Dean John L. Volakis

College of Engineering and Computing

This dissertation, written by Lamar Burton, and entitled Towards In-situ Based Printed Sensor Systems For Real Time Soil-Root Nutrient Monitoring and Prediction with Polynomial Regression, having been approved in respect to style and intellectual content, is referred to you for judgment.

We have read this dissertation and recommend that it be approved.

Nezih Pala

Wujie Wen

Arif Sarwat

Krish Jayachandran, Co-Major Professor

Shekhar Bhansali, Co-Major Professor

Date of Defense: March 27, 2020

The dissertation of Lamar Burton is approved.

Dean John L. Volakis

College of Engineering and Computing

Andrés G. Gil

Vice President for Research and Economic Development and Dean of the University Graduate School

Florida International University, 2020 
(C) Copyright 2020 by Lamar Burton All rights reserved. 


\section{DEDICATION}

To all of my family, for their love, endless support, and encouragement. 


\section{ACKNOWLEDGMENTS}

This dissertation would not have been completed without the support of my advisor, committee, colleagues, friends, and of course, my family.

First of all, my sincere appreciation goes to my co-major professors, Dr. Shekhar Bhansali and Dr. Krish Jayachandran, who offered me the opportunity to be in the great family of the Bio-MEMS Group as well as the Soils Lab. Their motivating personalities and endless encouragement improved my abilities in the early stages of research. In addition, they gave me great freedom to explore and learn to do independent research, and helped me grow as a researcher over the course of my graduate studies.

I would also like to show my gratitude to my committee members, Dr. Nezih Pala, Dr. Wujie Wen, and Dr. Arif Sarwat, for their support, insightful comments, and constructive suggestions.

I am grateful to all the members of the Bio-MEMS Group for creating a wonderfully collaborative work environment. I would also like to thank the staffs of the Electrical and Computer Engineering Department for their great commitment to student services.

I am extremely grateful to have met Dr. Sonja Montas-Hunter and I am very thankful for her support throughout this journey.

A very special gratitude goes out to the National Science Foundation (NSF) for providing partial research funding. I also acknowledge the McKnight doctoral fellowship from the Florida Education Fund and the CREST CAChe fellowhip at FIU. All this would be impossible without their generous support.

Finally, last but by no means least, I want to dedicate the dissertation to my family, for their love and for fostering all my academic endeavors, and to my father, whom passed along the this great journey. Thank you all for your love and support. 


\author{
ABSTRACT OF THE DISSERTATION \\ TOWARDS IN-SITU BASED PRINTED SENSOR SYSTEMS FOR REAL TIME \\ SOIL-ROOT NUTRIENT MONITORING AND PREDICTION WITH POLYNOMIAL \\ REGRESSION \\ by \\ Lamar Burton \\ Florida International University, 2020 \\ Miami, Florida \\ Professor Shekhar Bhansali, Co-Major Professor \\ Professor Krish Jayachandran, Co-Major Professor
}

This dissertation explores how to increase sensor density in the agricultural framework using low-cost sensors, while also managing major bottlenecks preventing their full commercial adoption for agriculture, accuracy and drift. It also investigated whether low-cost biodegradable printed sensor sheets can result in improved stability, accuracy or drift for use in precision agriculture. In this dissertation, multiple electrode systems were investigated with much of the work focused on printed carbon graphene electrodes (with and without nanoparticles). The sensors were used in two configurations: 1) in varying soil to understand sensor degradation and the effect of environment on sensors, and 2) in plant pod systems to understand growth. It was established that 3) the sensor drift can be controlled and predicted 2) the fabricated low-cost sensors work as well as commercial sensors, and 3) these sensors were then successfully validated in the pod platform. A standardized testing system was developed to investigate soil physicochemical effects on the modified nutrient sensors through a series of controlled experiments. The construct was theoretically modeled and the sensor data was matched to the models. Supervised machine learning algorithms were used to predict sensor responses. Further models produced actionable insight which allowed us to identify a) the minimal amounts of irrigation 
required and b) optimal time after applying irrigation or rainfall event before achieving accurate sensor readings, both with respect to sensor depth placement within the soil matrix. The pore-scale behavior of solute transport through different depths within the sandy soil matrix was further simulated using COMSOL Multiphysics. This work leads to promising disposable printed systems for precision agriculture. 


\section{TABLE OF CONTENTS}

CHAPTER

PAGE

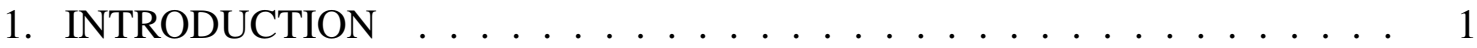

1.1 Research Background and Motivation . . . . . . . . . . . . . . . 1

1.2 Research Goals and Objectives . . . . . . . . . . . . . . . . . 2

1.3 Dissertation Organization . . . . . . . . . . . . . . . . . 3

2. SOILS IN AGRICULTURE $\ldots \ldots \ldots \ldots \ldots$

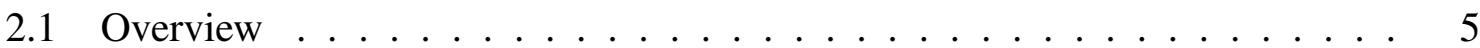

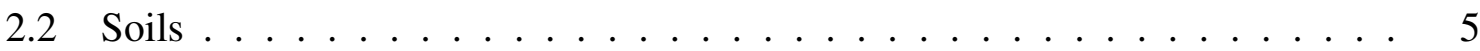

2.2 .1 Soils in Agriculture . . . . . . . . . . . . . . . . . . . . . . 6

2.3 Summary . . . . . . . . . . . . . . . . . . . 12

3. THE REAL-TIME REVOLUTION FOR IN-SITU SOIL NUTRIENT SENSING:

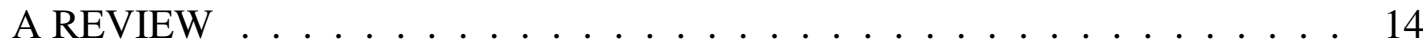

3.1 Overview . . . . . . . . . . . . . . . . . . . . . . . . . . . . . . 14

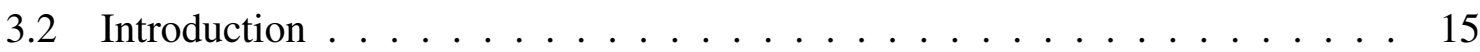

3.3 Soil Nutrient Sensors _ . . . . . . . . . . . . . . . . . . . . . . . . . 18

3.3 .1 Optical Sensors . . . . . . . . . . . . . . . . . . . . . . . . . . . . . . . . . 19

3.3.2 Electrochemical Sensors: . . . . . . . . . . . . . . . . . . . . . . . . . 24

3.4 Commercial Sensors . . . . . . . . . . . . . . . . . . . . . . . . . . . . . . . . . . . . . . 29

3.5 IoT Soil Nutrient Sensors _ . . . . . . . . . . . . . . . . . . . . 31

3.6 Machine Learning . . . . . . . . . . . . . . . . . . . . . . . . 36

3.6 .1 Supervised Learning . . . . . . . . . . . . . . . . . . . . . . . 37

3.6 .2 Unsupervised Learning . . . . . . . . . . . . . . . . . . . . . . . . . . . . 39

3.7 Summary $\ldots \ldots \ldots \ldots \ldots \ldots$

4. IMPLEMENTING PRINTING: A LOW-COST LARGE VOLUME MANUFAC-

TURING TECHNOLOGY $\ldots \ldots \ldots \ldots \ldots \ldots \ldots$

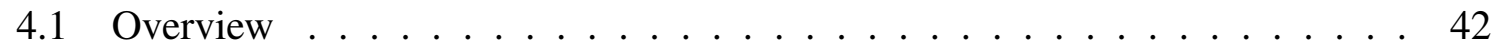

4.2 Printed Technologies . . . . . . . . . . . . . . . . . . . 42

4.2 .1 Types of Printing . . . . . . . . . . . . . . . . . . . . . . . 44

4.3 Printed Electronics and Challenges . . . . . . . . . . . . . . . . 52

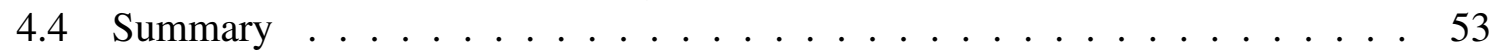

5. UNDERSTANDING THE ROLE OF SOIL HETEROGENEITY ON NUTRIENT SENSOR ACCURACY AND DRIFT $\ldots \ldots \ldots \ldots \ldots \ldots$

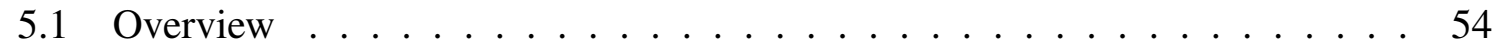

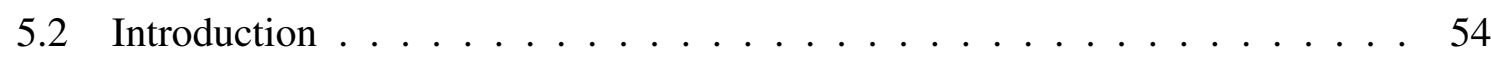

5.3 Materials and Methods . . . . . . . . . . . . . . . . . . . 56

5.4 Results and Discussion . . . . . . . . . . . . . . . . . . . . . 60

5.4.1 Infiltration and Percolation Measurements . . . . . . . . . . . . . . 62 
5.4.2 Effects of Soil Moisture . . . . . . . . . . . . . . . . . . . . . . . 64

5.4 .3 Effects of Soil Organic Matter . . . . . . . . . . . . . . . . . 67

5.4 .4 Effects of Soil Particle Size . . . . . . . . . . . . . . . . . . . . . . 69

5.4.5 Effect of Sensor Placement and TAM Measurements . . . . . . . . . . 70

5.5 Lead Length Measurements . . . . . . . . . . . . . . . . . . . 70

5.6 Modeling the effects of SR . . . . . . . . . . . . . . . . . . 73

5.7 Summary . . . . . . . . . . . . . . . . . . . . . . 74

6. FABRICATION OF SENSOR SHEETS AND PODS . . . . . . . . . . 76

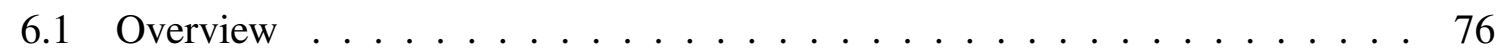

6.2 Introduction . . . . . . . . . . . . . . . 76

6.3 Materials and Methods . . . . . . . . . . . . . . . . . . . . . . 79

6.3 .1 Electropolymerization . . . . . . . . . . . . . . . . 80

6.3 .2 Potentiostat Circuit . . . . . . . . . . . . . . . . . . . 81

6.4 Sensor Pods . . . . . . . . . . . . . . . . . . . . . . . . . . . . 82

6.4 Overview .......................... 82

6.4 .2 Introduction . . . . . . . . . . . . . . . . . . . . 83

6.5 Materials and Methods . . . . . . . . . . . . . . . . . . . . 87

6.5 .1 Materials . . . . . . . . . . . . . . . . . . . 87

6.5 .2 Methods . . . . . . . . . . . . . . . . . . . . . . 88

6.5.3 On-chip plant phenotype analysis . . . . . . . . . . . . 92

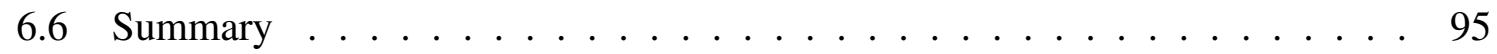

7. RESULTS AND DISCUSSIONS . . . . . . . . . . . . . . . . . 97

7.1 Overview . . . . . . . . . . . . . . . . . . . . . 97

7.2 Sensor Sheets Results and Discussion _ . . . . . . . . . . . . . . 97

7.2 .1 Sensor Sheets . . . . . . . . . . . . . . . . . . . . . . . . . . . 97

7.2 .2 Sensor Sheets Summary . . . . . . . . . . . . . . . . . . . . . . 104

7.3 SensePod Results and Discussion . . . . . . . . . . . . . . . . . 105

7.3 .1 SensePod . . . . . . . . . . . . . . . . . . . . 105

7.4 SensePod Summary . . . . . . . . . . . . . . . . . . . . . . . . . 109

8. CONCLUSION AND FUTURE WORK . . . . . . . . . . . . . . 112

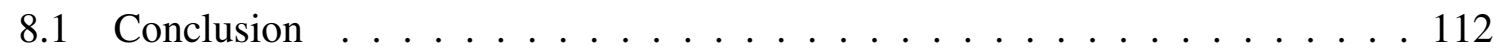

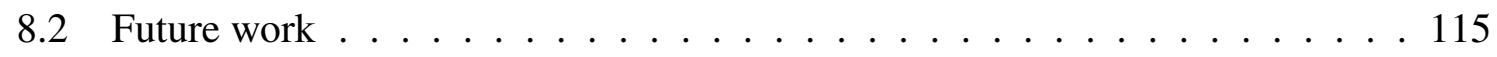

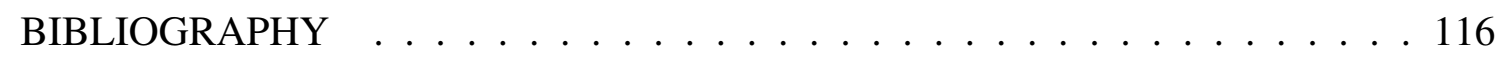

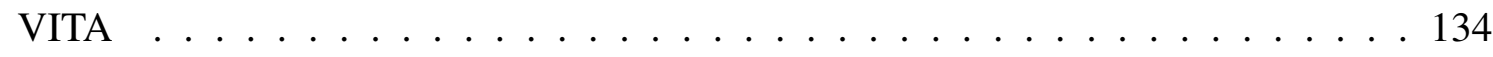




\section{LIST OF TABLES}

TABLE

PAGE

3.1 Types of soil nutrient sensors . . . . . . . . . . . . . . . . . 19

3.2 Compound bond and wavenumber . . . . . . . . . . . . . 21

4.1 Typical methods for fabricating sensors which include the silicon wafer process, roll-to-roll printing and ink-jet printing. The typical electrode thickness, resolution, printing speed that can be achieved for each method is also listed in addition to cost per meter and image carrier type. 43

5.1 Predictor attributes in SR model . . . . . . . . . . . . . . . . . 60

5.2 Trendline fitting in $2 \mathrm{~mm}$ Sandy Soil SR model . . . . . . . . . . . . 66

5.3 Algorithm Evaluation in SR model . . . . . . . . . . . . . . . 74

$6.1 \quad$ Plant chip devices for phenotype analysis . . . . . . . . . . . 96

7.1 Comparison of $\mathrm{NO}_{3}^{-}$Sensors . . . . . . . . . . . . . 103

7.2 Nitrate leachate levels of three lime stone-derived udorthents soil samples during irrigation cycle (LOW: 5-40 ppm, MED: 40-100 ppm, HIGH:

$100-300 \mathrm{ppm})$ as predicted by the IoT soil sensor network . . . . . . 103

7.3 Comparison of plant growth parameters when grown using Sense Pod and conventionally. For the experiment Red Crimson Clover seeds were used with each treatment group containing 10 seeds. . . . . . . . . . . 109 


\section{LIST OF FIGURES}

FIGURE

PAGE

2.1 As shown in the illustration, different portions of sand silt and clay make up corresponding textural classes. For example, a soil with $\% 70$ sand, $\% 10$ clay, and \%20 silt is considered sandy loam soil. This type of soil is popular in areas of South Florida. Shown in the triangle, each class is identified by a certain color inside the textural triangle. . . . . . . . .

2.2 The composition of soil is illustrated. Mineral and organic make up the solid fraction while soil and water represents the pore space of soil. The composition of soil greatly influences the soil texture, porosity, and structure. . . . . . . . . . . . . . . . .

3.1 Illustration of a flow chart for real-time soil nutrient sensors used in precision agriculture. . . . . . . . . . . . . . .

3.2 Illustration of IR spectroscopy. As incident light is directed to molecular bonds between two atomic masses, the energy is either absorbed, transmitted, or reflected. . . . . . . . . . . . . . . . . .

3.3 A simple illustration of ATR spectroscopy. As an IR beam is directed into the ATR device it is reflected from a series a reflectors which direct the incident light towards the ATR crystal. The crystal, in contact with a soil sample, reflects the light, creating an evanescence. The reflected energy exits the ATR crystal it is directed towards a spectrometer to produce a reflectance spectrum for the sample. . . . . . . . . . . .

3.4 Illustrated above is a TN prototype from An, X. et al. for detecting total $\mathrm{N}$; b) comparison between predicted and measured values of soil TN content [23]. . . . . . . . . . . . . . . . . .

3.5 Illustration of various types of commercial sensing applications. These include on-the-go mapping technologies which require the use of heavy farm vehicles; Point of use sensors which are usually handheld ion selective electrodes; Lab analysis units which are strictly used in lab facilities, and lastly the in-situ sensor which is only capable of moisture and pH measurements. . . . . . . . . . . . . . . .

3.6 a) Solar powered IoT sensor garden from Y. Mekonnen et. al. The IoT farm system also features sensor dictated irrigation [38]; b) nitrate calibration response using fabricated in situ nitrate ISE [37]; c) Layout of the IoT enabled soil sensor sheet system from L. Burton et al.; d) Real-time soil moisture content data used to correlate with in situ soil nitrate sensor; . . 32

3.7 Schematic of a typical IoT sensor system for real-time monitoring. . . . . . 33

3.8 Schematic of a decision management system for nutrient fertilizers. . . . . . 35

3.9 Illustration algorithms used in machine learning. [59] . . . . . . . . . . . 36 
4.1 Illustration of a pie chart which details the relative market share of printed sensors in 2025. [64] . . . . . . . . . . . . . . . .

4.2 Illustration of a bar graph detailing the compound annual growth rate for printed sensors between years 2015-2025. [69] . . . . . . . . . . . . . 44

4.3 Illustration of flexoprinting. Reprinted from [70] with permission from Elsevier. . . . . . . . . . . . . . . .

4.4 Illustration of a typical gravure printing process which includes the components. . . . . . . . . . . . . . . .

4.5 Illustration of a flat bed screen printing process of silver top electrodes. Reproduced from [70] with permission from Elsevier. . . . . . . . . . . 48

4.6 Illustration of a rotary screen printing of PEDOT. Reproduced from [70] with permission from Elsevier. . . . . . . . . . . . . . . 48

4.7 Illustration of ink-jet printing techniques. This includes the a)continuous drop technique, b)thermal technique, and c) the piezoelectric ink-jet printing technique. . . . . . . . . . . . . .

4.8 Illustration of hot lamination process. Reproduced from [70] with permission from Elsevier. . . . . . . . . . . . . . . . . . .

4.9 Illustration of lamination techniques. a) UV lamination where the UV reactive adhesive is applied to via printing technique and b) cold and hot lamination technique. Here the adhesive is applied to the laminate via print method and nip cylinders provide heat to cure the adhesive for hot lamination. [70]

5.1 Illustrates 15 sensors in a $3 \times 5$ matrix controlled by 8 reed relays. The relays for each column were connected to the working electrode of the same column and the relays for each row were connected to the reference and counter electrode. Setting the relay for a column and row to high allowed for a measurement to be taken from the working electrode while the counter and reference electrode were made from the corresponding common connection. Each relay was controlled using an Arduino mega controller. Each pin from the Arduino was connected to the base of a transistor, having VCC to its collector and an emitter connected to the relay. This allowed the microcontroller to control the relay while protecting the pins from kickback current given by the relays. A freewheel diode was also connected in parallel to each relay to protect the circuit from relay kickback current. . . . . . . . . . . . . . .

5.2 Calibration plot for N-doped PPy sensor; peak current vs nitrate concentration $(1 \mu \mathrm{Mol}$ to $60 \mu \mathrm{Mol}) \ldots \ldots \ldots$ 
5.3 As an example, the illustration is a COMSOL plot of Se (the effective saturation) as a function of the height of the $50 \mathrm{~mL}$ tube $(0$ is the base and 0.075 is the top of the tube). An initial saturation $\left(\theta_{s}\right)$ of 0.18 , and residual $\left(\theta_{r}\right)$ saturation of 0 . Each curve represents a different time. As time approaches infinity, the medium becomes saturated at the base of the tube (because there is a no flux boundary condition there. . . . . . . .

5.4 Illustration of infiltration and percolation of 18 percent moisture applied to sand column. The sand column was composed of quartz sand which contained electrodes positioned at three depths throughout the sand profile. The green circle at e1 TAM1 represents the initial infiltration of the conductive solution. This was also observed at e 2 TAM1. Percolation is shown from TAM1 to TAM3 as e1, e2, and e3 responded to the flowing conductive solution flowing through the sand matrix. e1 at TAM . . . .

5.5 Illustration of $\theta_{m}$ effect on SR at three different nutrient concentrations. The moisture ranges from $0 \%$ (dry) to $28 \%$ (field capacity). Observe accuracy increases as moisture level rises. . . . . . . . . . . . .

5.6 Illustratio of how OM influences the SR between ranges of $0 \%$ to $28 \%$ thet $a_{m}$ in two different particle size sands. . . . . . . . . . . .

5.7 Illustration reveals the variation in SR values as a result of particle size. More than $70 \%$ drift in SR was observed in smaller particle sized sand.

5.8 Illustration of SR values at various electrode depths (e1, e2, and e3) and time after moisture is applied to soil (TAM1, TAM2, and TAM3.e1 was placed at $11 \mathrm{~mm}$, e 2 at $25 \mathrm{~mm}$, and e 3 at $44 \mathrm{~mm}$. Graphs indicate at initial moisture application (TAM1) SR values were only observed in region e1. However, in TAM2 and TAM3, SR become more accurate with moisture content near zones e2 and e3.) . . . . . . . . . . . 7

5.9 Illustration of peak current vs the lead length in feet. . . . . . . . . . . 72

6.1 a) Layout of the IoT enabled soil sensor system. b) Seedling pellets on nitrate sensor sheet . . . . . . . . . . . . . . 78

6.2 Linear sweep potentiostat circuitry . . . . . . . . . . . . . 82

6.3 a) An illustration of the fabrication process; b) illustration of germination wells with integrated sensors and PDMS sheets; c) view of the sensor integrated germination pod device facilitating the growth of the legume plant ; d) zoomed view of legume roots housed in sensor pod device. . .

6.4 Flow diagram illustrating the multiplexed plant sensing devices communication to cloud, smartphone, and personal computer . . . . . . . . 990 
6.5 Illustration of real-time circuit setup which features a microcontroller interfaced with a mux for switching between plant sensor devices and an ORP which performs the measurements. All electrical components are connected using a breadboard. Data can be viewed on the serial monitor or sent to the cloud using LoRa. . . . . . . . . . . . . . . 91

6.6 Images of pod with integrated printed micro sensors and legume plants. . . . 92

7.1 SEM micrographs of (a) cross section of the electropolymerized working electrodes. Electrodeposition of pyrrole for varying time durations (b) 5 (c) 10 (d) 15 (e) 20 minutes at $100 \mu \mathrm{A} \ldots$. . . . . . . . . . . . 99

7.2 Soil leachate studies: (a) Differential pule voltammetry (DPV) response of sensors to varying nitrate levels (b) Current response of E5,E10, and E15 to nitrate levels $(10-200 \mathrm{ppm}) \ldots \ldots . \ldots 100$

7.3 Illustration of Au nanoparticles deposited on Carbon electrode. . . . . . . . 101

7.4 Illustration of Au electrode fabricated in clean-room (left) and an SEM image of Au electrode with Au nano-particles (right). . . . . . . . . . . . 102

7.5 Nitrate leaching in limestone-derived udorthent soil samples . . . . . . . . . 104

7.6 Cyclic voltammogram of fabricated sensors, $\mathrm{Gr}-\mathrm{Ag} / \mathrm{AgCl}$ silver in $1 \mathrm{mM}$, $2 \mathrm{mM}$, and 5 millimolar ferrous solutions. . . . . . . . . . . 106

7.7 Cyclic voltammogram of the doped PPy sensors in $1 \mathrm{mM}$ of NaNO3- and $\mathrm{H} 2 \mathrm{PO} 4$ electrolyte solution at a scan rate of $25 \mathrm{mV} / \mathrm{s} \ldots 107$

7.8 Calibration plots for nitrate (left) and phosphate (right) doped PPy sensors with peak currents vs concentration. . . . . . . . . . . 108

7.9 Illustration of nutrient uptake by legume plant over a span of approximately 7 days with fresh nutrients recharged after day five. Sensors were integrated into microreactor root chambers and immersed in agar nutrient media. . . . . . . . . . . . . . . . 108

7.10 The use of SensePod to monitor plant root and shoot growth over a period of seven days. . . . . . . . . . . . . . . . . . 110

7.11 a) Microscopic image of leaf epidermis from legume plant grown in the device, revealing normal stoma development and function. The red circles encompass an example stomata. b) microscopic image of leaf epidermis from conventionally grown legume plant. . . . . . . . . . . . 110 


\section{LIST OF ABBREVIATIONS}

ATP

ATR

CAGR

CHEMFET

CPS

CV

DOS

DPV

EC

EMF

EP

EPA

FET

FIA

GC

HAB's

IoT

IRS

ISE

ISFET

$\mathrm{K}$

$\mathrm{kNN}$

LL

LOC

LoRa
Adenosine Triphosphate

Attenuated Total Reflectance

Compound Annual Growth Rate

Chemically modified field effect transistor

Cyber-Physical System

Cyclic Voltametry

alinomycin-bis(2-ethylhexyl) sebacate

Differential Pulse Voltametry

Electric Conductivity

Electromotive Force

Electropolymerization

Environmental Protection Agency

Field effect transistor

Flow injection analysis

Gas Chromatography

Harmful Algal Blooms

Internet of Things

Infrared Spectroscopy

Ion Selective Electrode

Ion Selective Field Effect Transistor

Potassium

k-Nearest Neighbours

Lead Length

Lab-on-a-chip

Low power long range radio 


\begin{tabular}{|c|c|}
\hline MCL & Maximum Contaminant Level \\
\hline MEMS & microelectromechanical system \\
\hline MIP & Molecular imprinted polymer \\
\hline ML & Machine Learning \\
\hline $\mathrm{N}$ & Nitrogen \\
\hline NO3 & Nitrate \\
\hline NP & Nano particles \\
\hline NPOE & o-nitrophenyl octyl ether \\
\hline NRCS & National Resources Conservation Service \\
\hline $\mathrm{OM}$ & Organic Matter \\
\hline ORP & Oxidation reduction potentiometer \\
\hline$P$ & Phosphorus \\
\hline PDMS & Polydimethyl siloxane \\
\hline PPy & Polypyrrole \\
\hline $\mathrm{PVC}$ & polyvinyl chloride \\
\hline PVP & poly N-vinylpyrrolidone \\
\hline Redox & Reduction-oxidation \\
\hline RMSE & Root Mean Squared Error \\
\hline SEM & Scanning Electron Microscopy \\
\hline SMS & Short messaging system \\
\hline SVM & Support Vector Machine \\
\hline TAM & Time After Applied Moisture \\
\hline TDDA & Tetradodecylammonium \\
\hline TRRS & Time Resolved Raman Spectroscopy \\
\hline USDA & United States Department of Agriculture \\
\hline Vis MIR & Visible Mid Infrared \\
\hline
\end{tabular}


Vis NIR

WSN
Visible Near Infrared

Wireless sensor network 


\section{CHAPTER 1}

\section{INTRODUCTION}

\subsection{Research Background and Motivation}

Changing climate patterns, redistribution of water due to damning of rivers, and changing rainfall patterns have resulted in water becoming a scarce and limited resource for agriculture. This coupled with intense harvesting cycles have also contributed to a depletion of soil fertility. Farmers have attempted to address soil nutrient depletion through the use of excessive fertilizers, however, this has led to increased number of algal blooms, fish kills, and contamination of scarce water sources through runoff and leaching. Hence, the movement for precision agriculture which considers water, nutrients, reducing pollution, and ensuring that the water supply down the riverbed is potable. Current stateof-the-art sensing in precision agriculture incorporates only one sensor node per acre. It has been postulated that individual plant monitoring can lead to a quantum efficiency in both reduction of fertilizers, water usage and improving plant yield production. Hence, this dissertation investigates a new class of in-situ sensors and germination systems to support this goal. Achieving sensor density has not been possible because 1) increasing sensor density leads to drift and variability in sensing and 2) understanding how to interconnect such large area sensors remains a challenge. This dissertation provides a

fundamental framework for developing this new class of sensors by investigating architectures that allow large scale connectivity and understand how to calibrate drift issues of large scale in-situ soil nutrient sensing systems.

In-situ based soil sensors have become one of the worlds most critical technological infrastructures for precision agriculture, whose accuracy and reliability are essential for continuous monitoring, control, and the implementation of decision management systems. However, the response integrity of the in-situ based electrochemical sensors is 
compromised by naturally occurring disturbances found within the soil matrix and environment, including for instance, soil moisture, particle size, organic matter, sensor placement within the matrix and time of measurement. These disturbances contributes towards measurement uncertainty and eventually leads to failure of the in-situ based sensor platforms.

\subsection{Research Goals and Objectives}

The major objective of this dissertation is to obtain low-cost and high density soil nutrient sensor platforms for precision agriculture.

The envisioned objective was accomplished through the following goals:

- A. To fabricate and understand the behaviours of disposable sensors when used in-situ for real time nutrient analysis

- B. Utilize supervised machine learning for predicting the in-situ sensor response

- C. To use these fabricated disposable sensors in a three dimensional plant germination pod systems

The first goal was achieved by fabricating the disposable sensors, then creating an experimental controlled system to evaluate those sensors. After evaluation, the data was analysed. The system was also modeled and was compared to gain further insights on the viability of the models. The second goal was achieved by incorporating the fabricated sensors into the constructed germination pods and monitoring physical and chemical characteristics of the legume crops that were grown into the pod device. By addressing these challenges we anticipate to gain knowledge which aids in producing low-cost process and platform that lends itself to large-scale manufacturing and soil nutrient analysis. Such in-situ soil sensor platforms and plant sensor device will prove promising in precision agriculture and plant phenomics research. 


\subsection{Dissertation Organization}

In this dissertation we explore and investigate low-cost electrochemical sensing platforms for real-time in-situ nutrient analysis. These platforms were employed in both sandy soil and germination pod structures.

- Chapter one introduces and describes the format of the dissertation.

- Chapter two introduces soil and the effect of its composition on agriculture. These topics include soil water mobility, solute transport, and the impact of of soil physical and chemical characteristics on plant quality. It also discusses the state-of-theart in evaluating the soil physical and chemical parameters in laboratory settings.

- Chapter three presents a review on conventional sensors used in agriculture and introduces all measurement techniques used for soil nutrient analysis. It also discusses the state-of-the-art in monitoring and managing soil nutrients. The chapter also discusses the role of internet of things (IoT) and machine learning (ML) as revolutionary technology for soil quality monitoring and managing in precision agriculture. Finally, the limitations of the current technology and ways the device can be improved are discussed.

- Chapter four discusses low-cost large volume manufacturing technologies for producing soil nutrient sensors. Such technologies include ink-jet printing and laminating. To validate these techniques, standard lab micro fabricated sensors were used as controlled.

- Chapter five presents an experimental chapter which describes a lab method for understanding the effects of soil heterogeneity on the accuracy and drift of the fabricated disposable sensor platforms. Quartz sand consisting of three different particle sizes, four different organic matter percentages, and three different nutrient concentrations were selected to reveal the relationship between soil moisture 
content and nutrient sensor response. Here, classification based ML techniques were also implemented to develop a correction factor equation to help account for sensor drift. In this chapter we also use multi physics to simulate the movement of nutrients through sandy soil. This study helps to identify optimal sensor placement within the soil matrix and best time for taking a sensor measurement after irrigation is applied.

- Chapter six presents the fabrication of sensor sheets and pod systems and discusses the integration of electronics with these systems. It also discusses the fabrication and characterization of nitrate $\left(\mathrm{NO}_{3}^{-}\right)$doped polypyrrole (PPy) ion selective electrodes (ISE's). It highlights the role of electropolymerization (EP) time and recipe concentration on the membrane film growth and performance. Scanning electron microscopy (SEM) technology was used to observe surface morphology and characterize the sensor device.

- Chapter seven presents the results and discussions of the fabricated sheet and pod systems and entails the results from the study of the legume crops grown inside the sensor integrated pod devices.

- Chapter eight concludes and summarizes all the work within the dissertation. This chapter identifies the limitations of the research presented in this dissertation and discusses the possibilities of future work. This includes the possibilities of translating the printed sensing platforms onto additional substrates such as agricultural fabrics. The reliability, mechanical integrity of the substrates and perform adhesion quality experiments using industrial scratch test instruments are considered. 


\section{CHAPTER 2}

\section{SOILS IN AGRICULTURE}

\subsection{Overview}

Low-cost soil nutrient sensor technology has been sought by farmers to help revolutionize precision agriculture, especially for assessing soil quality in real-time. Before much insight is given on the subject of such sensors, an exploration of soils is needed in order to further understand soil behavior and its influences on in-situ sensor readings. In this chapter, soils, their functions and properties are discussed. This also includes soil water, as the transport of water in soils greatly influences the fate of nutrients such as nitrates. Understanding the properties and behaviors of soils allows the production of theoretical models that explain solute movement through soils and enables key research questions to be answered regarding the performance of in-situ based soil quality sensors and their placement within the soil profile. This is extremely important with sensor systems whichwhich are effected by properties such as moisture and humidity

\subsection{Soils}

Soils are an essential part to all life on earth and its properties and quality directly effects the nature of plant ecosystems and the capacity of soil to help sustain all life and society [1]. Soils support and supplies nearly all food sources except those harvested from oceans, and has become increasingly important for the production of biomass feedstocks for fuels, manufacturing, and livestock. One popular postulation is that the dependency on soils to produce food, fibers, fuels, inks, and biodegradable plastics will

increase drastically to meet the growing demand. As the human population increases by billions in such a short time, the demands for these products will also increase. In con- 
trary, the amount of soil available to produce these products will not increase. Therefore, it is imperative that we better our understanding and management of soils to ensure that all species sharing life, both on and within soils on earth, continue to thrive.

\subsubsection{Soils in Agriculture}

In any ecosystem, soils perform six key functions: 1) supporting the growth of grasses and plants by serving as a nutrient bed medium for plants and roots; 2) controlling the fate of water in a hydrologic system such as water loss, utilization, contamination, and purification; 3) serve as a recycling system for dead and decaying materials; 4) provide a habitat for living organisms; 5) influence the condition of the atmosphere by taking up and releasing gasses, dust, and heat energy to the air; and finally 6) serve as an engineering medium for human needs such as transportation and housing. These jobs carried out by soils are extremely complex and share more complex relationships with the many soil properties and characteristics.

Soil physical properties are texture, structure, porosity, chemistry and color. Soil texture refers to the size of particles that make up a soil and is separated into three categories: sand, silt, and clay (Figure 2.1). A typical loam soil is made of $45 \%$ mineral particles, $5 \%$ organic matter, $20-30 \%$ air and $20-30 \%$ water (Figure 2.2 ). The manner in which sand, silt, and clay are arranged is defined as the soil structure. This can be commonly seen as aggregates of roundish granules, cube-like blocks, flat plates, and other shapes. Soil chemistry is usually considered as the nutrients and $\mathrm{pH}$ of the soil. Since soil is negatively charged, nutrients with positive charges such as potassium and ammonium are attracted and are not loss due to leaching. However, the negative charged nutrients such as nitrate are not attracted to the organic and mineral matter and moves quite easily through the soil matrix of mos soils. Soil colors can range from brown, black, red, 


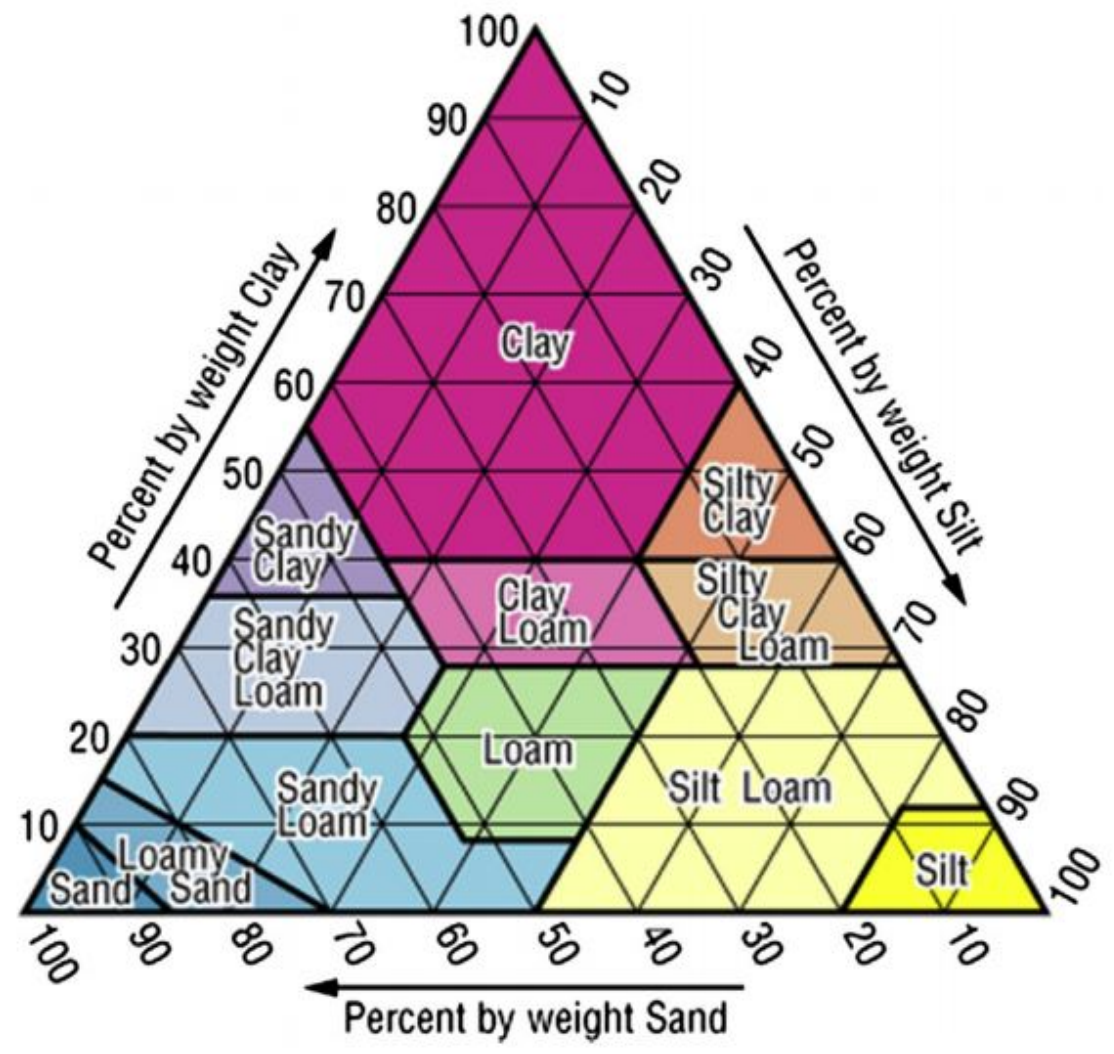

Figure 2.1: As shown in the illustration, different portions of sand silt and clay make up corresponding textural classes. For example, a soil with $\% 70$ sand, $\% 10$ clay, and $\% 20$ silt is considered sandy loam soil. This type of soil is popular in areas of South Florida. Shown in the triangle, each class is identified by a certain color inside the textural triangle.

and white and are closely associated with organic matter and iron content. These colors can also indicate many behaviors of the soil. One example would be soil of light blue in color which indicates poor drainage. These physical properties greatly influences the function of soils and how they can be managed [1].

\section{Soil Water}

Soil water is essential for many ecological functions of soil including the survival and growth of plants and soil organisms. Soil water is held within the pore spaces of soils 


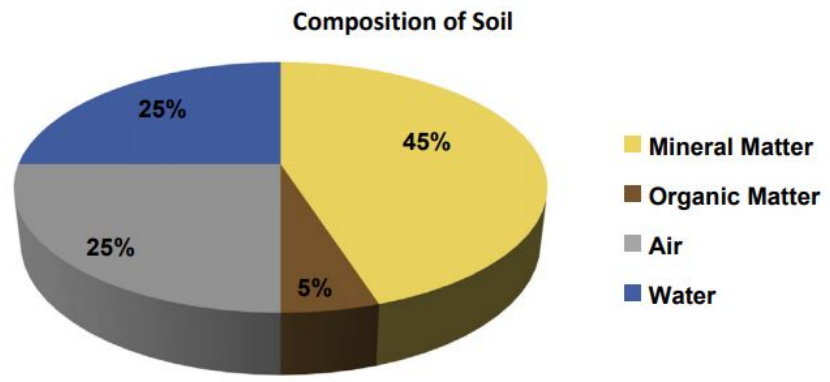

Figure 2.2: The composition of soil is illustrated. Mineral and organic make up the solid fraction while soil and water represents the pore space of soil. The composition of soil greatly influences the soil texture, porosity, and structure.

and contains hundreds of dissolved organic and inorganic substances and nutrients. This can also be considered the soil solution. Since water molecules in soil exhibit polarity, this explains how the molecules are attracted electrostaticly to ions and colloidal surfaces. The hydrogen bonding of water and neighboring air molecules within the soil matrix accounts for water movement and retention in soils. This phenomenon can be further described as capillarity. Below are subsections containing brief descriptions of key behaviors related to soil water.

Capillarity Capillarity is known as the upward movement of water and is caused by two distinct forces: adhesion/adsorption, and cohesion. Cohesion is defined as the attraction of water molecules to each other while adhesion is defined as the attraction of water molecules for solid surfaces.

Gravitational and Pressure Potential Gravitational potential is another phenomenon that explains how water may move through soil. The gravitational potential can be written as:

$$
\psi_{g}=g h
$$


where $\mathrm{g}$ is the acceleration due to gravity and $\mathrm{h}$ is the height of the soil water above a reference elevation within the soil profile.

Volumetric/mass Water Content Volumetric water content, or $\theta_{V}$, is defined as a specific volume of water associated with a given volume of soil $\left(\mathrm{m}^{3}\right)$. This can also be expressed as the mass water content or $\theta_{M}$, which equates to a given mass of water directly associated with a specific mass of soil.

Water Flow in Soil There are three types of ways in which water moves through soil; saturated flow, unsaturated flow, and vapor movement. Saturated flow, or percolation, happens as soil pores are completely saturated with water. This usually occurs during or after a heavy rainfall or irrigation event. In this matter, Darcy's law can be used to describe the quantity of water per unit of time flowing through a saturated porous media such as a saturated column of sandy soil. This can be expressed as follows:

$$
\frac{\partial \epsilon \rho}{\partial t}+\nabla \times \vec{u}=0
$$

Where $\epsilon$ is the porosity (amount of empty space) of the porous medium (for a saturated medium, this also corresponds to the volume of fluid), $\rho$ is the fluid density and $\vec{u}$ is the velocity vector which described flow through the porous medium -commonly referred to as the Darcy velocity.

The Darcy velocity is a function of the pressure $(\mathrm{p})$ the permeability $(\mathrm{K})$ of the porous structure, the fluid's dynamic viscosity $(\mu)$ as well as the gravitational force. This is represented in the second term of (2.3) where $\mathrm{g}$ is the acceleration of gravity and $\nabla \mathrm{D}$ is the gradient of elevation and indicates the direction of the vertical coordinate.

$$
\vec{u}=-\frac{K}{\mu}(\nabla p+\rho g \nabla D)
$$

The parameters $\epsilon$ and $\mathrm{K}$ are properties of the porous medium. These may be obtained experimentally or numerically. One useful model for $\mathrm{K}$ is the Kozeny-Carman equation 
which approximates the permeability as a function of the porosity $\epsilon$ and average particle diameter $\mathrm{d}_{p}$

$$
K=\frac{d_{p}^{2}}{180}\left(\frac{\epsilon^{3}}{\left(1-\epsilon^{2}\right)}\right)
$$

For soil samples containing mixed particle sizes, $\mathrm{d}_{p}$ can be replaced with an average particle size. However, even though theoretically, porosity is a material property, mixed samples may have a different porosity due to sorting and cementation.

Unsaturated flow appears when smaller pores hold and transmit water as a result of larger pores in soil being filled with air. This type of movement of water in soils takes place majority of the time. Given the macro-pores are completely filled with air, the water must flow to smaller pore spaces within the soil matrix. As the water content and potential may vary, this type of water flow in soil becomes difficult to measure. Unlike saturated flow which relies on gravity, the matrix potential gradient is the key force behind water flow in unsaturated soils. Matric potential gradient is the difference of matric potential of moist soils to nearby dry soils where water will move. In other words, in unsaturated soils water will flow from higher moisture films to that of lower moisture films. Another important event which can be modeled is that of infiltration. Infiltration is the process where free-water, such as irrigation, rainfall, and snow-melt, enters the soil pore spaces from the surface and becomes soil water. This is known as infiltrability and can be written as follows:

$$
i=Q / A \times t
$$

where $\mathrm{Q}$ is the volume quantity of water $\left(\mathrm{m}^{3}\right)$ infiltrating, $\mathrm{A}$ is the area of the soil surface $\left(\mathrm{m}^{2}\right)$ exposed to infiltration, and $\mathrm{t}$ is the time in seconds. Once the water has infiltrated the surface of the soil, it then moves downward through the profile. This process is called percolation and appears in both saturated and unsaturated soils. Another important behavior of soil water is that of field capacity. This occurs after rainfall or irrigation and water has percolated into matrix and has completely filled the micro-pores. Unsatu- 
rated flow will allow water movement, however, at a much slower pace due to capillary forces.Field capacity allows the maximum amount of water useful for plants while providing sufficient air in macro pore spaces. This allows aeration for aerobic microbiota and plants.

Vapor movement occurs as vapor pressure changes in relatively dry soils and can be categorized into two types, internal and external. Internal vapor movement occurs within the soil matrix, and external occurs near or at the surface soil as surface evaporation. Vapor movement is influenced by water, salt, and temperature. Vapor moves from high moisture to low moisture, low salt content to high salt content, and from higher temperature soils to lower temperatures. As vapor moves upward from higher temp soils towards cold atmosphere, the vapor can then condense to form dewdrops within the soil matrix.

Modeling Water and solute flow in soils In addition to the models listed above, solute flux, $J_{s}\left[M T^{-1} L^{-2}\right.$ have been traditionally modelled as advection, diffusion, and dispersion. Advection is considered the solute particle movement with water flux and can be written as;

$$
J_{c}=J_{w} c
$$

where $\mathrm{c}\left[\mathrm{M} L^{3}\right]$ is the solute concentration in the solution and, in saturated or in unsaturated soil, the water flux $J_{w}\left[\mathrm{~L} T^{1} L^{2}\right]$ is the Darcy or the Buckingham-Darcy flux [2]. Molecular diffusion is considered the thermal motion of molecules at temperatures above absolute zero and is responsible for the random movement of particles resulting in a gradual mixing of the solute. This solute flux, $J_{d}\left[M T^{1} L^{2}\right]$ is described using Ficks first law and and can be written as:

$$
J_{d}=-\theta D_{m} \nabla c
$$


where $\mathrm{D}_{m}$ is considered the molecular diffusion coefficient tensor in free water. Lastly, the hydrodynamic dispersion or spreading of solute relies solely on the structure of soil (i.e. the particle size, pore size, flow path length, velocity within pores and mixing between pores) [2]. This is written as:

$$
J_{h}=-\theta D_{h} \nabla c
$$

where $\mathrm{D}_{h}$ is considered the coefficient tensor of hydrodynamic dispersion. Overall, by summing the three parts, the total solute flux can be derived as:

$$
J_{s}=J_{w} c-\theta D \nabla c
$$

where D has resulted in the combination of dispersion and diffussion coefficent into a single coefficient $\mathrm{D}=\mathrm{D}_{h}+\mathrm{D}_{m}$.

\section{Soil Organic Matter}

Organic matter (OM) exists in all soils but in most soils the percentage is minimal. Nevertheless, the presence of this organic matter is highly important. In fact, majority of the cation exchange and water holding capacity in soils is provided by soil organic matter. The cation exchange capacity allows the OM to retain plant nutrients and act as slow release fertilizer systems for plants. Given this ability, OM plays a key role in maintaining soil quality.

\subsection{Summary}

In summary, soils are a diverse and heterogeneous habitat with characteristics and properties that influence their functions in the ecosystem and especially agriculture. Their properties, texture, structure, porosity, chemistry and color, directly effects the fate of 
its nutrients and soil water. Most importantly, soil moisture has the propensity to transport free moving ions such as nitrates. This can be done in both saturated and unsaturated soil conditions but more so in unsaturated soils. This movement through the surface and throughout the soil profile can be explained as infiltration and percolation. The movement can be further modeled by some derivation of Darcy's Law. By improving the general understanding of soils and soil solute transport, theoretical models can be deduced to aid in enhancing the management practices of water and nutrients. One example would be modeling percolation through sandy soil profiles over-time. Such model can aid in determining optimal placement of in-situ based sensors and optimal time for taking measurement after infiltration. 


\section{CHAPTER 3}

\section{THE REAL-TIME REVOLUTION FOR IN-SITU SOIL NUTRIENT SENSING: A REVIEW}

\subsection{Overview}

Globally, farmers are seeking advanced precision technology to help transform their practices into a more sustainable and productive agri-tech process []. As changing weather patterns, climate change, and unprecedented natural disasters threaten food production, accurate and real-time soil data detailing the condition of farmland has become one of the most valuable resources among farmers. This chapter presents an overview of in-situ based soil nutrient sensors for precision agriculture and current machine learning techniques applied to smart agriculture. Especially, for real-time soil nutrient monitoring. Real-time soil sensor data can be exploited in manners that increase farm production and profit, maintain and increase product quality, promote food security, and ensure environmental protection. Section 2.2 discusses the need for adopting real-time sensing technology for agriculture and environmental monitoring. Section 2.3 discusses available sensing methods for soil nutrient analysis, current sensors on the market, and those materials needed for self fabrication. Researchers have already attempted to develop real-time in-situ soil nutrient sensors based on optical and electrochemical techniques. Of these sensor systems, only a few of them are commercially available for monitoring. Section 2.4 defines IoT and identify various applications of IoT sensors for precision agriculture. Section 2.5 describes several key machine learning algorithms, both classification and regression, that are successfully used in smart farming. Based on the literature presented in this chapter, there still exists a need to understand the effects of soil heterogeneity on the analytical performance of both electrochemical and optical systems when used insitu. By doing so, these sensors can be fully adopted as suitable commercial platforms. 
Overall, these sensors harness the potential to revolutionize decision management systems in agriculture as internet of things (IoT) soil nutrient sensors.

\subsection{Introduction}

Over the past six to seven decades, during the green revolution, farmers have met the growing global demand for food using technologies, methods, and regulations that are inefficient, unsustainable, and non-ecofriendly [3]. Currently, farmers are using these methods to produce more than three billion metric tons of produce per year [4], feeding a global population of approximately 7.8 billion. In the next thirty years, Earth's population will reach approximately 9 billion, and will require an increase in food production by more than 70 percent [5]. To meet this challenge of successfully increasing yield production, the exploitation of agrochemicals and nutrient fertilizers, as much as 190 million metric tons per year [6], has remained the number one method of choice of farmers. However, given the harmful environmental impact due to the excessive and poor management of fertilizer application, agriculture requires the use of advanced technology in order to continue meeting food demands (present and future), while reducing the environmental impact [3]. The agri-tech revolution is emerging and aims to use advanced precision technology, such as real-time soil nutrient sensors and IoT, to meet the future demands for food, fiber, and fuel [6], in a more sustainable, efficient, and eco-friendly manner. 


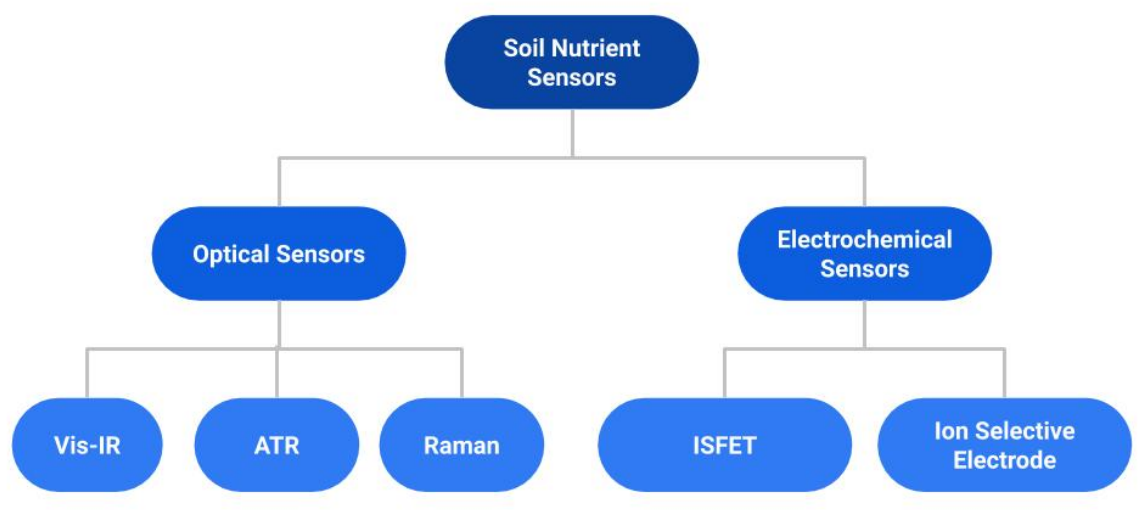

Figure 3.1: Illustration of a flow chart for real-time soil nutrient sensors used in precision agriculture.

Precision agriculture provides remotely sensed data which allows farmers to minimize resource input and maximize yield. Precision agriculture is a data demanding system that allows the farmer to assess the heterogeneity of soil physical and chemical content, geospatial variations, and crop data, for optimizing resource utilization based on acquired field data [7] [8]. Precision information and advancing technology have given rise to innovative smart decision management systems, such as sensor dictated irrigation. Real-time soil moisture profiles have allowed farmers to derive optimal fertilizer rate application and harvest time. However, the efficiency of decision management systems relies on the rapid accuracy of technology and cost (per sensor/sample) [9]. There has been a significant rise in research and development of precision agriculture technologies to monitor $\mathrm{pH}$, salinity, moisture content, organic matter, and texture, however, in situ monitoring of soil macronutrients, Nitrogen $(\mathrm{N})$, Phosphorus (P), and Potassium (K) remain a challenge [10].

$\mathrm{N}, \mathrm{P}$, and $\mathrm{K}$ are known to be responsible for assimilating proteins, synthesizing metabolic energy, and producing adenosine triphosphate (ATP). They are considered the most es- 
sential macronutrients. Although they naturally exist in many forms throughout the soil environment, these nutrients are often exploited by humans in the form of fertilizers. Variations of soil nutrient and chemical composition are found spatially throughout agricultural farms and natural environments. These nutrient variations range from depleted soils to excessively fertile soils. As nutrients become limited, plants respond by reducing growth and altering their aspects for nutrient acquisition, utilization and morphology to maximize and acquire the limited resources [11]. When excess nutrients are available, those macro-nutrients which are not consumed by plants are leached into ground and surface waters during rainfall and irrigation events. Surface water uses mainly impacted by nutrient pollution include municipal and private water supplies, recreational waters for swimming and boating, cold and warm water fisheries, agricultural water supplies, and navigational waters. It has been noted that the accumulation of these nutrients in waters has harmful effects on humans [12] and biodiversity [13], examples include cancer-causing drinking waters and decreased marine species population due to eutrophication. In addition, substantial economic loss and costs have been associated with excessive anthropogenic nutrient loading and harmful algal blooms (HAB's). Declining fishing and boating activities as a result of nutrient pollution and HAB's, has caused over $\$ 1$ billion in losses each year to tourism. The EPA has also expressed their encouragement for states to immediately prioritize management actions for nutrient pollution in watersheds and water bodies [14]. Thus, due to the impact of excess soil nutrients, there is a need for an affordable system that can continuously and accurately monitor soil conditions and movement of their macronutrients in real-time.

Traditional soil nutrient measurement practices employ the random grid sample technique to obtain soil cores. The cores are then packaged and transported to a laboratory. Next, the process will require expensive lab instrumentation and additional extractant solutions for further chemical analysis. These practices can include Kjeldahl wet diges- 
tion, dumas combustion, and gas chromatography (GC) mass spectrometry. In addition, ion-selective electrodes and ion-selective field-effect transistors are also used in the laboratory but their potential for in situ analysis in field settings will be further discussed in a later section. Although the lab methods are highly accurate, they lack the ability to deliver continuous real-time analysis of farm soil. They are also challenged with time per sample and cost per sample, two major challenges encountered by large scale farmers seeking to map their terrain. With additional emphasis, farm terrain is variable, meaning what is present on one farmland may not be the same for another within the terrain. Therefore, accurate, high-density, in situ monitoring of soil conditions, such as the ones listed in the subsequent sections, hold a great advantage over traditional lab methods.

\subsection{Soil Nutrient Sensors}

Advanced technology and communications are allowing faster and stable transmission of sensor data worldwide, and are becoming more affordable due to miniaturization and lower material costs. As shown in Table 3.1, the literature reveals various types of sensors, both commercially available and under development, to measure soil chemical properties [10], [13], [15]. These can be employed in either map-based [16] or real-time systems. Among all the available techniques, the majority of in situ soil sensing involves optical and, or, electrochemical methods (figure 3.1). In this review, optical and electrochemical sensors for detecting soil nutrients are highlighted. Optical sensors use spectroscopy based on reflectance spectroscopy to identify the magnitude of reflected and absorbed energy by soil nutrient ions. Electrochemical sensors for soil nutrient determination function by using ion-selective electrodes to initiate a current or voltage output that reflects the concentration of target ions. 
Table 3.1: Types of soil nutrient sensors

\begin{tabular}{llll}
\hline Sensor concept & Status of development & Current results & Key references \\
\hline Vis-NIR & Laboratory/field & Soil pH and nutrients & $(26-28)$ \\
Vis-MIR & Laboratory & Soil mineral nitrogen & $(24-25)$ \\
ATR Spec & Laboratory/field & Soil Nutrients & $(28)$ \\
Raman & Laboratory/field & Soil Nutrients & $(29)$ \\
ISE & Laboratory/field & Soil pH and nutrients & $(39-44)$ \\
ISFET & Laboratory/field & Soil pH and nutrients & $(6-7),(18)$ \\
\hline
\end{tabular}

\subsubsection{Optical Sensors}

Researchers are investigating the use of optic sensors for field measurements of soil nutrients in a rapid and nondestructive manner. Infrared spectroscopy (IRS) is a technique used to determine the structure of compounds, both inorganic and organic. This has been accomplished by using an ultraviolet, visible, and infrared polychromatic radiation source to quantify light energy absorbed and, or diffusely reflected by soil nutrients. To further understand the phenomenon of infrared spectroscopy, it is understand that a) IR spectroscopy is a technique that analyzes molecular vibrations, and b) the principle of IR spectroscopy is based on the theory of simple harmonic oscillation. An example of a simple harmonic oscillator system would be the shared bonds between two atoms. These spring-like bonds display resonant characteristics dependent upon the "spring" constant which describes the force between them, and the atomic weight at the end of each bond. Thus, the oscillation of a particular chemical bond coincides with a unique frequency and intensities of energy. Further, these unique frequencies of oscillation between bonds are complementary to particular wavenumbers $\mathrm{cm}^{-1}$ (table 3.2), sharing a positive correlation. As seen in figure 3.2, incident radiation is projected upon a sample at similar vibrational frequencies shared between the bonds. This radiation becomes adsorbed while others are intently reflected. A spectrophotometer records the reflectance, forming a reflectance spectra that reveals the magnitude of energy captured as a function of wavelength. Therefore, a molecule can be identified by comparing its absorp- 


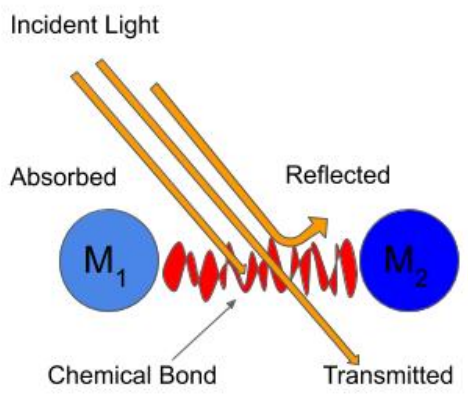

Figure 3.2: Illustration of IR spectroscopy. As incident light is directed to molecular bonds between two atomic masses, the energy is either absorbed, transmitted, or reflected.

tion peak to a database of spectrums. Some of these sensors have been designed to be highly portable and can offer a full spectrum in less than 1 second [17]. These sensors have also been integrated into microfluidic devices such as the one presented in [18]. The researchers produced a lab on chip integrated with a light emitting diode and photoresistor to measure the changes in absorption and detect levels of ammonia and amino acids. The microfluidic device was said to respond to a wide range of concentrations and posses a limit of detection as low as 2 parts per million.

Literature lists [19] [20] and explores [21] [22] [23] [24] [25] many soil properties such as moisture, organic matter, nutrients, and $\mathrm{pH}$, that can be identified rapidly and cost efficiently using diffuse reflectance spectroscopy. Investigations by [26] [27] explored the use and range of NIR for quickly measuring nutrients, then later employed the use of MIR to increase the accuracy of this system. Researchers in [28] highlighted the use of both Vis-NIR and Vis-MIR for soil nitrogen analysis, however, these measure- 


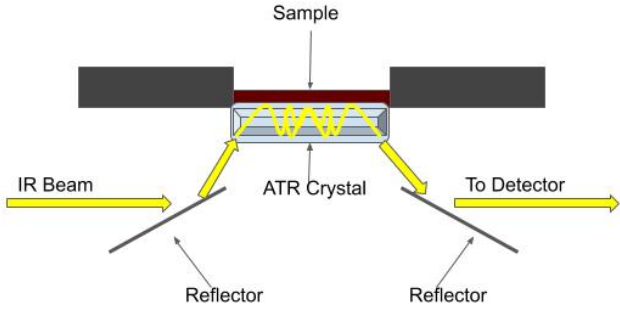

Figure 3.3: A simple illustration of ATR spectroscopy. As an IR beam is directed into the ATR device it is reflected from a series a reflectors which direct the incident light towards the ATR crystal. The crystal, in contact with a soil sample, reflects the light, creating an evanescence. The reflected energy exits the ATR crystal it is directed towards a spectrometer to produce a reflectance spectrum for the sample.

ments were made in a laboratory on prepared soil samples. In [29] [30], researchers explored the use of NIRS for on the go nutrient-sensing using soil penetrating probes. These probes transmit and receive light using fiber optic cables and optic aperture panes adjoined with soil. The sensor devices were interfaced with farm vehicles to map the variability of soil parameters while traversing the farm terrain.

Table 3.2: Compound bond and wavenumber

\begin{tabular}{lll}
\hline Compound & Bond & Wavenumber \\
\hline Alkenes & $\mathrm{C}=\mathrm{C}$ & $1610-1680$ \\
Aldehydes, ketones, esters & $\mathrm{C}=\mathrm{O}$ & $1680-1750$ \\
Acids (hydrogen bonds) & $\mathrm{O}-\mathrm{H}$ & $2500-3300$ \\
Alkanes, alkenes, arenes & $\mathrm{C}-\mathrm{H}$ & $2840-3095$ \\
Alcohols, phenols & $\mathrm{O}-\mathrm{H}$ & $3230-3670$ \\
Primary amines & $\mathrm{N}-\mathrm{H}$ & $3350-3500$ \\
\hline
\end{tabular}




\section{Attenuated Total Reflectance Spectroscopy}

Attenuated total reflectance (ATR) spectroscopy operates using the same principles of infrared energy absorption by particles and molecular bonds. However, instead of targeting samples with infrared spectrums and acquiring the diffuse reflectance, a crystal directly adjoining a target receives the incident IR energy (figure 3.3). The crystal is usually made of diamond, zinc selenide, geranium, and, or thallium iodide, however certain criteria should be considered before selecting the proper ATR crystal, such as the refractive index, spectral range, and physical and chemical properties of the sample. When incident radiation is directed upon the crystal, an evanescent field is created between the crystal and sample due to the incident reflection. The energy then departs the ATR crystal and travels towards a spectrometer. Here, a spectrum will be produced for the sample. Linker et al. used ATR in the 2,500-50,000 nm spectrum to identify nitrate from various types of soil pastes [31]. The use of ATRS for the detection of soil nutrients requires little sample preparation, however, there still is a great challenge minimizing the interferences caused by soil moisture and existing minerals. In addition, ATRS instrumentation is expensive and delicate, therefore it is not practical for use in field settings.

\section{Raman Spectroscopy}

Raman spectroscopy is a technique that involves measuring the changes in wavelength and intensity of scattered light upon interaction with a sample. Incident light energy is absorbed and reemitted from the sample at different frequencies known as the Raman scatter or Raman effect. The Raman spectrum from the observed sample is a unique fingerprint of a sample that offers information about the chemical structure and identity of a sample, the polymorphism, intrinsic strain, and sample contamination. In [32] researchers have explored and developed a portable Raman spectroscopy device and method which provided good results for the determination of phosphorus and other nu- 
trients in soils and vegetation. The device is also capable of detecting nutrients in both wet and dry soils and contains compartments for drying, sieving, and grinding soil samples. This eliminates further laboratory needs and provides in situ determination. The device also features an on-board microprocessor which allows the data acquired by the spectrometer to calculate the concentration of phosphorus within the sample.

The advantages of optical sensors are that they are a highly flexible form of analysis, capable of measuring irregular surfaces non-destructively and requires little to no sample preparation. Such an example is illustrated in figure 3.4. This method of measurement eliminates the need for additional sample solutions and preparations such as extractants, sample grinding, and drying. It has also been noticed that these reproducible measurements can be produced at an extremely low cost [15]. Since measurements are taken in a non-destructive manner [7] and they can be deployed subsoil for rapid in situ real-time analysis, this makes them perfect for in situ soil measurements. Collecting proper soil samples is a critical aspect of soil testing which must consider the spatial distribution, depth, and time of day. Therefore large scale deployment of these devices has the potential to offer a higher resolution of nutrient-sensing within the terrain.

Although these sensors are becoming increasingly popular and less expensive to assemble, they are still affected by many soil physical and chemical properties to different degrees [30]. While exploring MIR for soil nutrient sensing, [27] discovered that measurements in desiccated soils were extremely difficult. Later, [31] and [25] recommended soils be prepared moist and paste-like to facilitate adequate soil-crystal contact for improving results. A recent study by Viscarra revealed the effects of moisture content and the environment when deriving proximal sensed Vis-IR data. Researchers also state that in order to successfully implement in-situ NIRS, calibration is required for each site, which can potentially increase sample preparation time and cost. 
a)

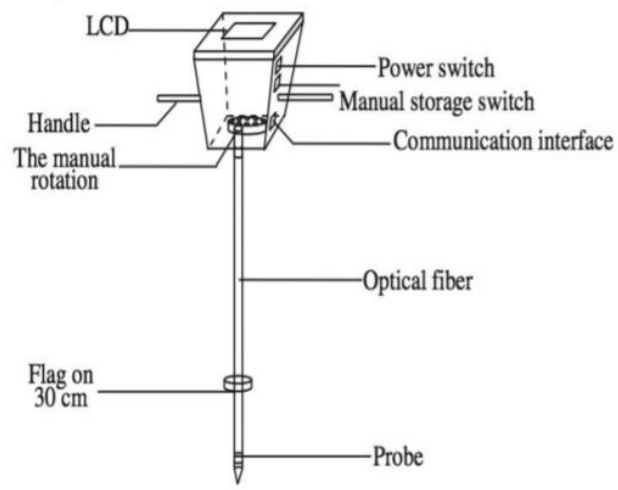

b)

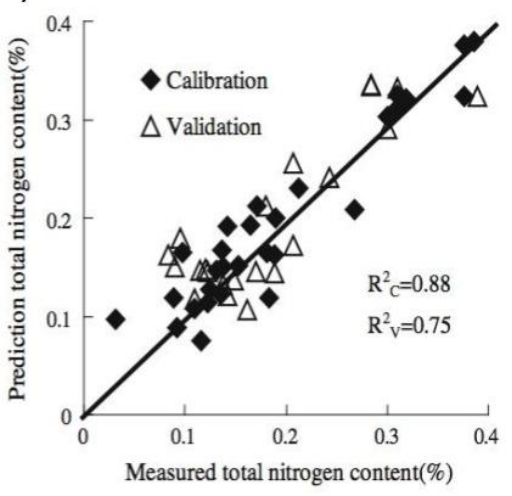

Figure 3.4: Illustrated above is a TN prototype from An, X. et al. for detecting total N; b) comparison between predicted and measured values of soil TN content [23].

\subsubsection{Electrochemical Sensors:}

Electrochemical sensors are a common approach to understanding the chemical content of a solution and are becoming increasingly popular due to their miniature size and ease of integration into autonomous systems. The electrochemical device functions by coupling a chemically selective layer, known as a recognition element, to an electrochemical transducer. When the recognition layer is in contact with the ion of interest, the transducer converts the chemical energy of the selective membrane interaction into an electrical signal. The electrical techniques used for the transduction of the target analytes allows electrochemical sensors to be further organized into subcategories. These categories include potentiometric, which measures the changes in membrane potential; conductometric for measuring changes in conductance; impedimetric sensors which measure changes in impedance, and amperometric sensors for measuring changes of current at the sensing membrane. The two most employed electrochemical sensors for determining soil nutrients are the ion-selective field-effect transistors and ion-selective electrodes. 


\section{Ion Selective Electrode}

Just as the name implies, ion-selective electrodes are conductive pieces of matter with a specialized membrane (glass/polymer) whose potential reflects the concentration of the selective target being measured. These ion concentrations are determined by calculating the voltage drop across the membrane using the Nernst equation. The Nernst equation is by far the most fundamental equations to the understanding of electrochemical cells. Formulated by German scientist Walther Nernst, this equation considers standard potential, temperature, and concentrations in an electrochemical reaction to relate with the potential of the working electrode.

The Nernst equation is depicted as:

$$
\phi=\phi^{0}+R T / z_{1} F\left(\ln a_{1}^{\text {solution }}\right)
$$

where $\phi$ is the electrode potential, $\phi^{0}$ is the standard value of the potential, $a_{1}$ is concentration and $z_{1}$ is the target ion charge number, $\mathrm{R}$ is the gas constant, $\mathrm{T}$ is the absolute temperature, and $\mathrm{F}$ is the Faraday constant. Usually, in a galvanic cell, the difference between two electrodes submerged in a solution is measured as the electromotive force (EMF). EMF is described as that source of energy which enables electrons to move around an electric circuit [33] and is determined by the difference of oxidizer and reducer potentials [34] in a reduction-oxidation (Redox) reaction. Under certain conditions, when the reference potential remains constant in a galvanic system and the active electrode follows the Nernst equation, the EMF equation can be described as:

$$
E=E^{0}+S \log a_{1}
$$

where $\mathrm{E}$ is the measured EMF, $E^{0}$ is the standard $\mathrm{EMF}$ value at $\mathrm{a}_{I}=1$, and $\mathrm{S}$ is the response slope. The slope is usually $\mathrm{S}_{25}=59.18 / z_{1} \mathrm{mV}$ at $25 \mathrm{C}$. However, according to 
literature the slope can differ with freedom of about $0.2-2 \mathrm{mV}$ [35]. The slope is derived by obtaining a calibration curve using at least 3 standards. Once this is done the ISE can be used to measure concentrations of target analytes in solutions. The sensitivity can be calculated by dividing the linear part of the calibration slope by the surface area of the working electrode.

ISE's are proven to have a wide dynamic range, appearing to distinguish between variations in concentrations of residual nitrogen $(0.1-10,000 \mathrm{ppm} \mathrm{N})$ and nutrients. ISE's are traditionally employed in the laboratory facilities to obtain nutrient measurements. A common approach for using ISE's are integrating them into microfluidic structures. Microfluidics allows the controlling of liquid samples at micro and pico levels while the integrated ISE's allows rapid analysis of nutrients. In [36], researchers presented a flow loop microfluidic device integrated with an ISE sensor for in-situ analysis of phosphate. Electrochemical sensors can be employed in a variety of environments. Literature reveals that majority of electrochemical sensors are used to detect nutrients, toxins, and pollutants in waters and aqueous solutions [37] [38] [39] [40] [41]. However, ISE's have also been known to gather rapid measurements in slurries, unfiltered soil extract and naturally moist soils [42], [43], and [9]. The portable multi ion measurement system developed in [42] allowed site specific nutrient analysis and characterization of the spatial variability of surface soil nutrients. Researchers in [9] produced a system for on-thego mapping of nutrients using direct soil measurement methods that allowed nutrient electrodes to distinguish between very high and very low concentrations. In addition to aqueous solutions, moist soils and slurries, electrochemical sensors have also been used to determine ionic concentrations in gaseous forms.

There has been numerous reports on various membrane materials and recognition elements used to electrochemically quantify soil nutrient concentrations. Though many recognition elements for nutrient detection exists, the most popular membranes are 
based on polyvinyl chloride (PVC)-ligand membranes and molecular imprinted polymers. Glass membrane based ISE's are popularly used for determining $\mathrm{pH}$, but not commonly reported for detecting nutrients. Some of the most popular membranes for detecting nutrients include the use of doped polymer membranes such as Nitrogen doped polypyrrole (N-doped PPy) for the detection of soil nitrogen, tetradodecylammonium onitrophenyl octyl ether (TDDA-NPOE) based electrodes for detecting nitrate, valinomycinbis(2-ethylhexyl) sebacate (DOS) based electrodes for detecting potassium, and cobalt rod-based electrodes for measuring phosphate [44]. Molecularly immprinted polymer (MIP) based membranes for detecting soil nitrogen has been explored in [45]. In [45] the electrochemical doping was used to fabricate a nitrate sensor. The researchers further increased the sensitivity of their sensor by doping polypyrrole nanowires. Optimal membrane compositions and preparation conditions have been determined for polypyrrole (PPy) for determining soil nitrate concentrations [46]. The researchers reported that te lifetime of the membranes can also be extended by adding appreciable amounts of plastisizers to the membranes. Overall, due to the ease of preparation, literature finds that MIPs and PVC are suitable for fabrication selective ISE's.

\section{Ion Selective Field Effect Transistor}

Ion-selective field-effect transistors (ISFET's) are simply ISE's joined with a field-effect transistor (FET) and can also be referred to as chemically modified field-effect transistors (CHEMFET's). The function and structure of a FET are quite interesting, as it uses an electric field within a region of the device to control the flow of current and is comprised of three terminals; the source, drain, and gate. There are two types of FET devices, p-type and n-type. The device type dictates which carrier conducts the flow of current. There are two carrier types, electrons, and holes. In an n-type FET, a gate receiving a positive voltage causes a carrier depletion region due to the repelling of holes 
in the region beneath the gate. As the holes are repelled, electrons are pulled from the substrate into the channel region, assembling a bridge between the source and drain. Now, as a voltage is applied between the drain and source, electrons can flow freely through this channel. Quite the opposite effect occurs within a p-type device, as a positive voltage will deplete the carriers and reduce the conductance. By applying voltage to the gate, an electric field can be generated to control the amount of charge in the channel and therefore influence the conductivity of the channel. This applied voltage is referred to as the threshold voltage, as it represents the number of volts required to sufficiently accumulate a quantity of electrons for a conductive channel. In the case of a CHEMFET or ISFET, the ISE membrane is appended to the insulating layer of the FET, thereby chemically modulating the threshold voltage as a function of the concentration within the solution.

The use of ISFET's with flow injection analysis (FIA) has been discussed in [8]. Researchers have investigated technology to successfully measure soil nutrients from prepared extracts. The system based on the multi-ISFET technology featured a rapid extraction ISFET system for real-time in-field measurements of soil nitrogen in less than 5 seconds. Literature has also discusses ISFET devices that have demonstrated the ability to analyze various soil nutrients [9], however, like many previous devices the sensor relies on a extracton system. It is reported that the flow injection analysis system helps to mitigate ISFET sensor drift and increase the efficiency and performance of the sensor system. Compared to ISE's, ISFET's are relatively smaller in dimensions, have a higher signal to noise ratio, and rapid response time.

\section{Disadvantages / Advantages}

Disadvantages of the ion-selective electrodes are that it requires frequent calibration [47], may require additional extraction solutions, and relies on soil moisture for accu- 
rate nutrient readings. Another disadvantage is that nutrient sensor response is affected by various properties of soil such as the textural class, particle size, volumetric water, organic matter, and interfering ions listed in Hofmeister's series [48]. Consequently, the limited success of these sensors are due to the measurement inconsistencies caused by the above disadvantages. Also, the effective use of electrochemical sensors for determining soil nutrients requires some type of procedure or device for nutrient extraction and rinsing agent for the electrochemical sensor which creates a further lengthy process for analysis. In addition to the sensor requiring periodic rinsing, it is recommended that the user have an understanding of soil texture and physical parameters for advanced calibration. Further, the development of highly selective membranes is needed, as this can also improve the accuracy of in-situ soil nitrate ISE's for nutrient determination.

Literature pertaining to this sensor system reveals that the ion-selective electrodes can make an ideal platform for in situ soil nutrient measurements. These soil sensor measurements can be acquired in a rapid manner with lab-grade accuracy [49]. The measurements can also be performed in slurry solutions, unfiltered extractants, and naturally moist soils. Additionally, electrochemical sensors can be fabricated using low cost materials, such as the ammonia sensor based on paper substrates reported in [50]. Further, printed sensor platforms can be mass-produced using common industrial printing techniques [51], then interfaced to a single microcontroller for real-time soil nutrient analysis [52], as shown in figure 3.6c.

\subsection{Commercial Sensors}

Over the past few years, much breakthrough has been made towards producing in-situ and point of use soil quality sensors. These revolutionary devices include the hand held ion selective electrodes and the tractor based sensors coupled with flow injection analy- 


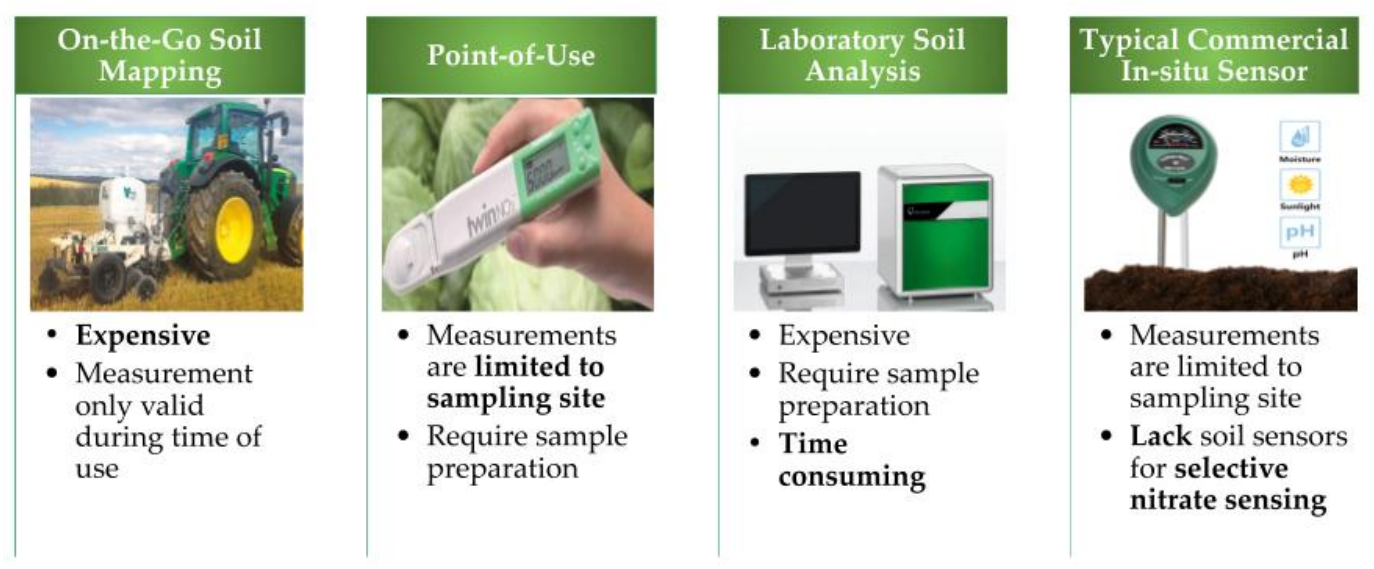

Figure 3.5: Illustration of various types of commercial sensing applications. These include on-the-go mapping technologies which require the use of heavy farm vehicles; Point of use sensors which are usually handheld ion selective electrodes; Lab analysis units which are strictly used in lab facilities, and lastly the in-situ sensor which is only capable of moisture and $\mathrm{pH}$ measurements.

sis systems(figure 3.5). Big companies such as John Deere, Veris Technologies, Horriba, and Caterpillar have already claimed stake in producing and providing these commercial devices to consumers. There are two major classes of methods for in-situ based soil nutrient quality monitoring, they are point-of-use and on-the-go methods. Point-of-use system are hand-held devices that encompasses the use of either ion selective electrode or optical based sensor for quick measurement in remote areas. The portable devices operates by displaying some digital readout which represents a nutrient concentration from micro-litre volumes of soil leachate extracts. Point of use sensors have been reported to perform and offer accurate measurements as comparable to those lab based instruments. The second class of method, on-the-go, also utilizes both electrochemical and optic based sensor for soil quality monitoring. However, the sensor devices feature global po- 
sition system and some flow injection analysis components which are affixed to some large farm vehicle. The on-the-go soil quality measurements are captured as the vehicle traverses the terrain while servicing the soil and crops for planting, pest management, and harvest. The GPS based system allows the user to create a map of terrain which displays a readout of the spatially varied soil condition throughout the farm land.

Limitations Due to the portable nature of the point-of-use systems, they are limited in terms of deployment density when attempting to interrogate soil quality in large areas. On-the-go systems produce measurements that are valid during time of use. This gives these system a disadvantage since farm terrain varies spatially and can undergo significant phisiochemical changes due to weathering and and irrigation. another limitation of both systems is cost. Due to this, even field deployable sensor networks have been limited to one sensor system per acre.

\subsection{IoT Soil Nutrient Sensors}

A system of devices or objects assigned unique identifier tags and exercises the ability to transmit and, or receive signal over a network can be referred to as the internet of things (IoT). an interesting concept behind this system is that its devices can function perfectly without human-to-human, or, human-computer interaction. The unique identifier which bridges devices to the cloud, giving the in situ soil nutrient sensor systems the advantage of real-time data visualization and sharing. The support of communication technologies such as short messaging system (SMS) for text messaging, WiFi internet, ZigBee, and LoRa low powered long-range radio modules have allowed innovative systems for control, prediction, and monitoring of many soil parameters. This is done in a manner where interaction between the sensor systems and soil can be handled via a web 


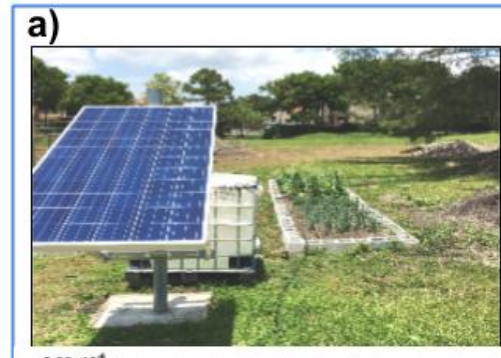

c)
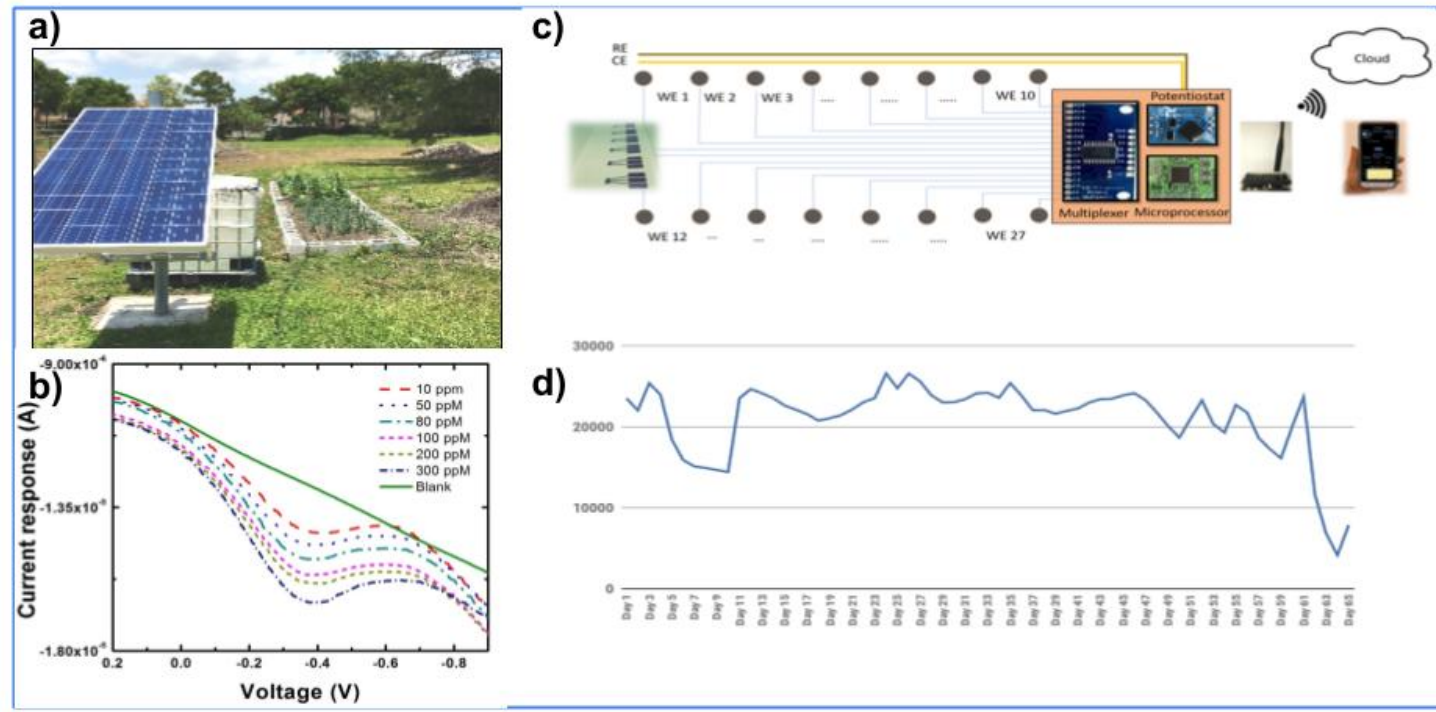

d)

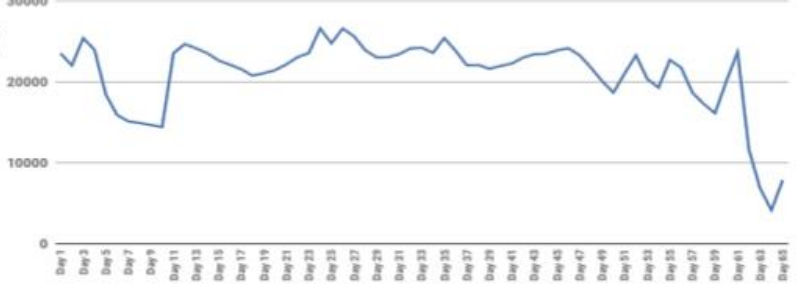

Figure 3.6: a) Solar powered IoT sensor garden from Y. Mekonnen et. al. The IoT farm system also features sensor dictated irrigation [38]; b) nitrate calibration response using fabricated in situ nitrate ISE [37]; c) Layout of the IoT enabled soil sensor sheet system from L. Burton et al.; d) Real-time soil moisture content data used to correlate with in situ soil nitrate sensor; 

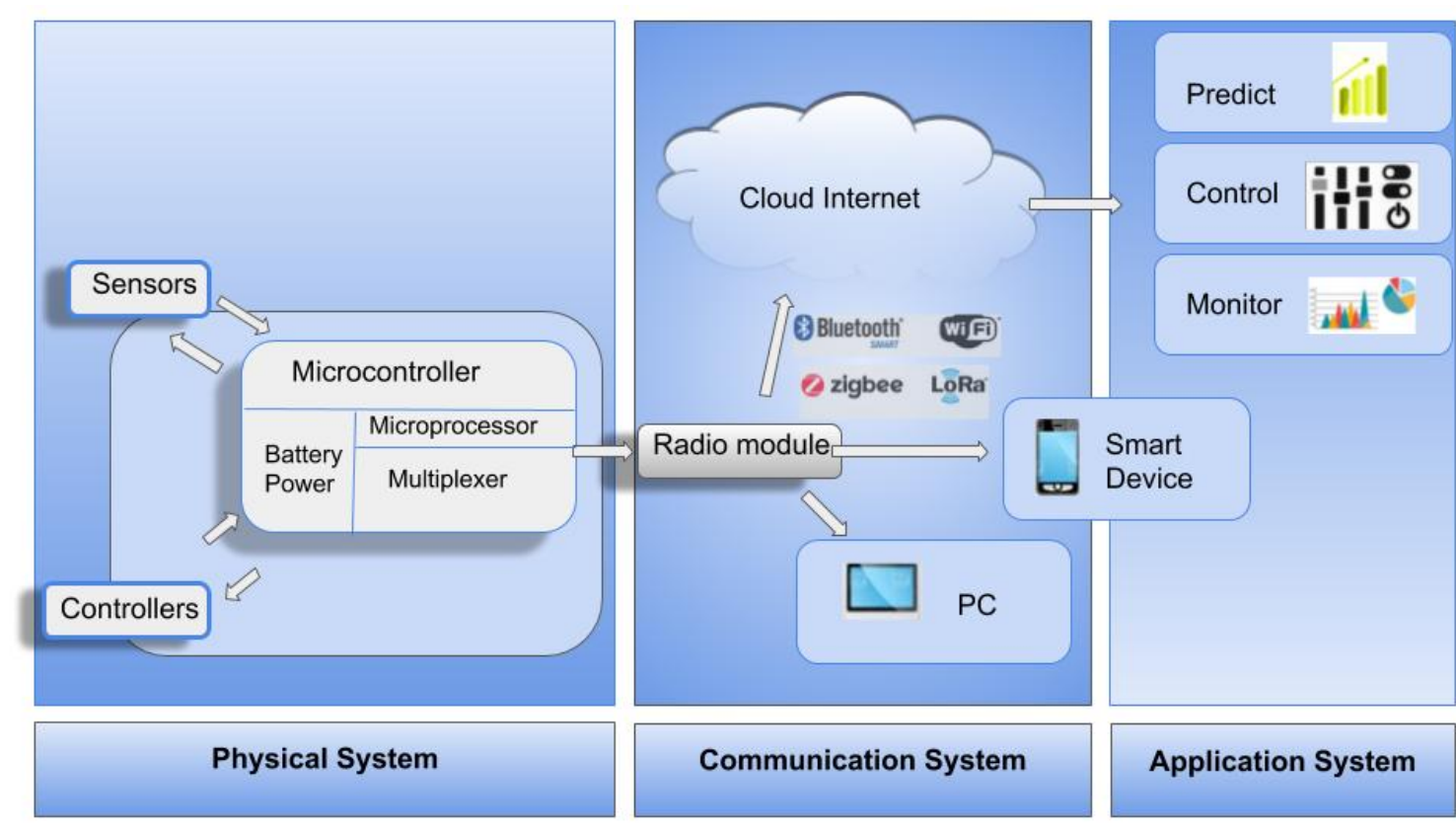

Application System

Figure 3.7: Schematic of a typical IoT sensor system for real-time monitoring.

application. As seen in figure 3.7, a typical IoT sensor system consists of a sensor interfaced with a microcontroller; the microcontroller relies on some battery source to power its function of commands from the microprocessor and is also interfaced with a radio module [53]. The radio module, which holds the unique numeric identification or IP address, transmits and receives data over a network to a cloud, smart device or personal computer. The personal computer and cloud web application can feature deep learning and algorithms for predicting yield and crop growth parameters. The microcontroler and wireless radio module configuration was also used in [54], where researchers deployed a wireless sensor micro heater network for smart agriculture using. In addition, real-time soil nutrient monitoring techniques have been used to improve crop cultivation and production efficiency in precision agriculture with the use of additional actuator systems for control, such as sensor dictated fertigation. Real-time, in-field, automated soil monitoring systems have been further explored in [52] [53] [55] [56].

Researchers in [55] developed a soil nutrient mapping system capable of rapidly assess- 
ing large-scale nitrate variations with lab-grade accuracy. The system is usually attached to heavy farm vehicles for mapping soil conditions as it traverses farm terrain. Later, they implemented validation tests of their nitrate extraction and measurement system. The authors found the level of agreement between the system's nitrate extraction subunit and standard laboratory measurements to be excellent.

In [52], smart gardening IoT soil sheets were developed and implemented in limestone derived Udorthent soils in south florid. The inkjet sensor printed paper sheets allowed real-time analysis and measurement data were transmitted via Waspmote, ZigBee, cloud server and mobile smart device. The author's latest explorations and further research aim to better identify influences of soil heterogeneity such as texture, organic matter, and moisture on the performance of the in situ polymer sensor systems.

Researchers in [56] developed a micro total analysis system and mobile microchip capillary electrophoresis device for field detection of nutrient extracts. The lab-on-a-chip (LOC) systems allowed fluid handling, activation, and observation of chemical reactions in the soil. Although the system is criticized for its lengthier sample and measurement time, compared to other on the go proximal sensor systems, the device produces measurement readings that are comparable to laboratory analysis at a much lower cost. Although not specifically intended for soil nutrient analysis, researchers in [57] employed an inexpensive sensing platform for in situ monitoring of water and nutrients that also provided decision management for a smart irrigation system. Perhaps future work would allow researchers to adopt methods for precision agricultural practices. In addition to their use in precision agriculture, a real-time in situ system allows site-specific monitoring of pollutants for environmentalists. Such site-specific data has the potential to improve and better enforce environmental legislature [15].

The interest of real-time in-situ sensors and IoT systems has increased in agriculture, especially as farmers demand greater decision management systems for improving yield 


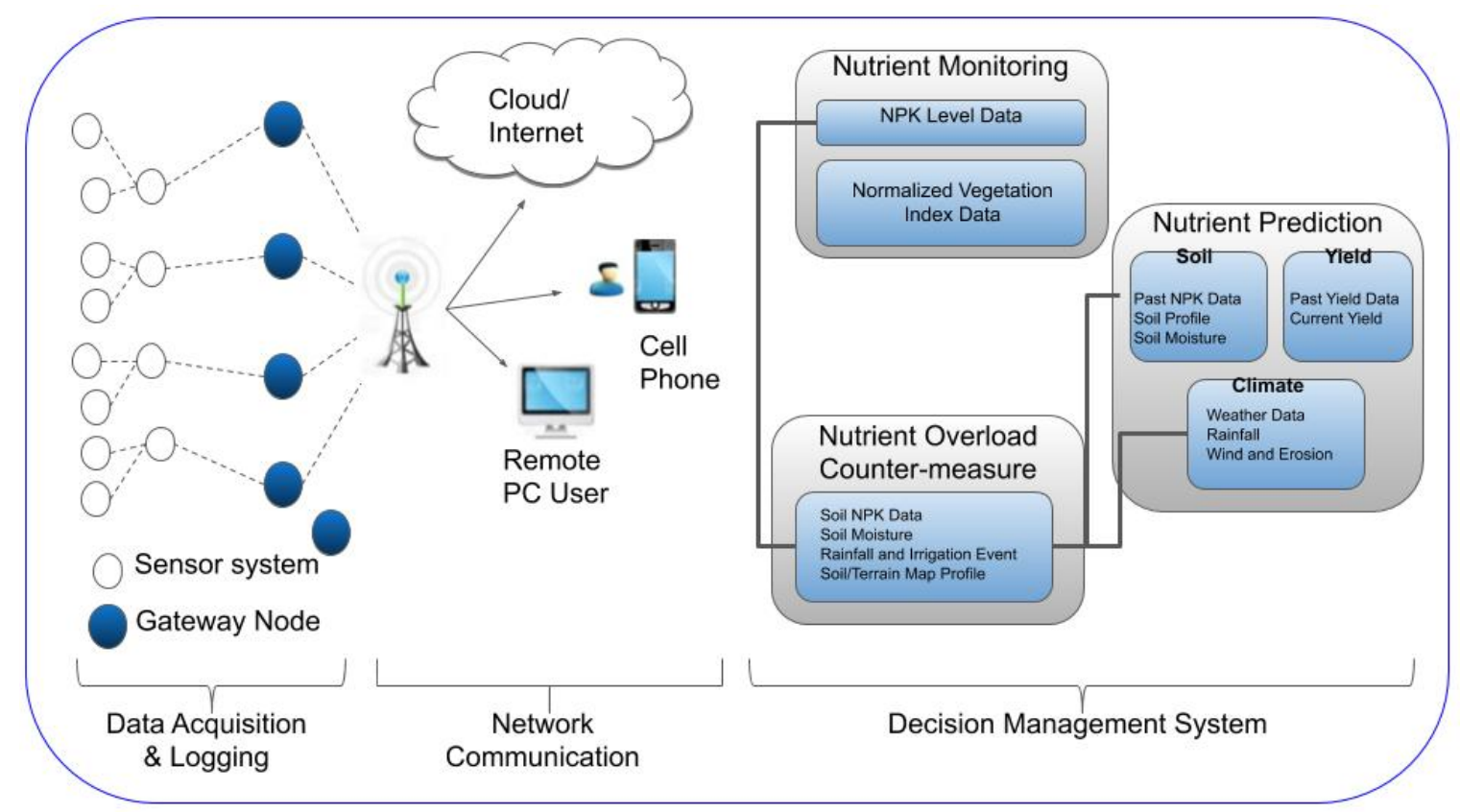

Figure 3.8: Schematic of a decision management system for nutrient fertilizers.

production and nutrient fertilizer application. A decision support systems incorporate various data elements such as climate, irrigation, crop genetics, energy, land terrain, human activity, and economic resources to provide an analysis of how their interrelations influence productivity. As shown in figure 3.8, a typical decision management system for nutrient application would include deployable sensor systems for acquiring real-time soil nutrient conditions. The sensor systems then forward the data to a gateway node, where the data can be logged or transmitted to a cloud, cellular or PC network. After this, the data can be further analyzed using machine learning, and neural networks to help predict soil nutrient requirements and present countermeasures for the overloading of nutrient fertilizers. The current state of the art decision management system, SMART Fertilizer Management, is a cloud-based software that offers nutrient requirement data for over 250 different crops. According to SMART, the platform helps farmers maximize yields an average of 15 percent, save costs and increase profits. 


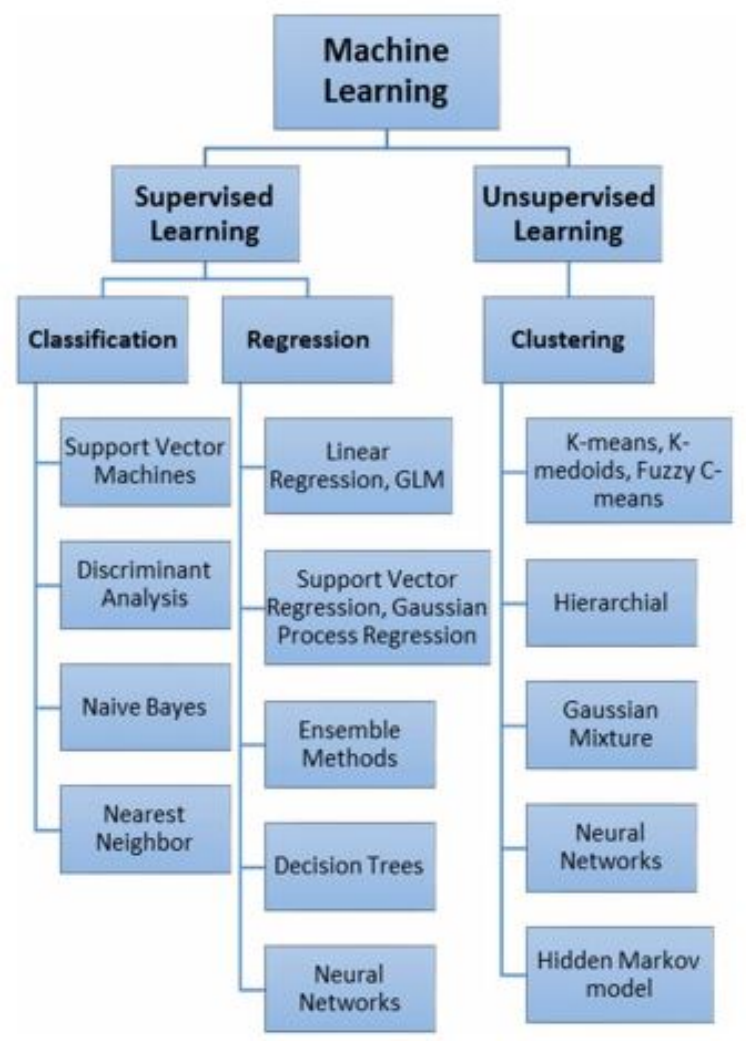

Figure 3.9: Illustration algorithms used in machine learning. [59]

\subsection{Machine Learning}

Machine learning uses statistical techniques and algorithms to detect patterns from large datasets [58]. As the training sets increase, so does the performance of the algorithms. ML approaches in agriculture allows farmers to autonomously manage farmland and solve problems using data from sensor networks. Current machine learning techniques can be categorized into two types, supervised and unsupervised learning (Figure 3.9). ML in precision agriculture is emerging and can currently be categorized in crop yield management, crop disease management, livestock management, soil management, and water management. Further discussion on the techniques of ML are detailed in the next sections below. 


\subsubsection{Supervised Learning}

In supervised ML, a known class of outputs in a training dataset is analyzed and used to develop a model which can predict new examples [60]. Mathematically, the overall goal is to identify the function $f: \mathcal{X} \rightarrow \mathcal{Y}$. This function is entirely based on a training set $\mathcal{A}_{n}=\left\{\left(\boldsymbol{x}_{1}, y_{1}\right), \ldots,\left(\boldsymbol{x}_{n}, y_{n}\right)\right\}$, which is composed of pairs of input and output points

$\boldsymbol{x}_{i} \in \mathcal{X}$ and $y_{i} \in \mathcal{Y}$. The derived model can be implemented for the precision agriculture data such as in farm soil monitoring and sensor drift detection and prediction. As shown in the figure 3.9, classification and regression are the two techniques commonly used in supervised ML. The difference between the two are that classification deals with qualitative variables while regression deals solely with quantitative variables. The illustration in figure 3.9 shows a list of algorithms commonly used by different techniques. The algorithm prescription depends mainly on the data type, size and expected outcome, but in many cases the trial and error approach for selecting algorithms is taken. Hence, selecting the best algorithm remains a one of the most challenging features for implementing machine learning.

Regression As a supervised ML technique, the main objective of regression is to predict continuous responses. This can include stock prices, fluctuations in the electrical demand, and time series sensor data [61]. Since the relationships between $\mathcal{X}$ and $\mathcal{Y}$ can be both linear and nonlinear, the algorithms themselves can also be either linear or nonlinear.

Linear Regression Linear regression is a simple approach to predicting the quantitative response of $\mathcal{Y}$ to a single predictor variable $\mathcal{X}$ [61]. as the name implies, it assumes there will be a linear relationship to the input and output variables, thereby mathemati- 
cally this can be written as:

$$
\mathcal{Y}=\beta_{0}+\beta_{1} \mathcal{X}
$$

where $\beta_{0}$ and $\beta_{1}$ are constants representing the intercept and slope and together can be considered as as the coefficients or parameters.

Multi-linear Regression When more than one predictor exists, it is more appropriate to predict the response by using multi linear regression. This ids accomplished by asserting each predictor a separate slope coefficient in a single model. The multiple linear regression model can then be depicted as:

$$
\mathcal{Y}=\beta_{0}+\beta_{1} \mathcal{X}_{1}+\beta_{2} \mathcal{X}_{2}+\ldots \beta_{P} \mathcal{X}_{P}
$$

Polynomial Regression In many cases, the relationship between $\mathcal{Y}$ and $\mathcal{X}$ is not linear and therefore must be described using a non linear approach. Polynomial regression extends the linear model by adding extra predictors, obtained by raising each of the original predictors to a power [61]. The polynomial function can then be writen as:

$$
\dagger_{1}=\beta_{0}+\beta_{1} x_{i}+\beta_{2} x_{i}^{2}+\beta_{3} x_{i}^{3} \ldots \beta_{d} x_{i}^{d}
$$

K-Nearest Neighbor K-Nearest neighbor (KNN) is one of the most simplest algorithms used in both classification and regression based supervised ML(CITE). The goal of $\mathrm{K}$-nearest is to predict the distribution of $\mathcal{Y}$ given $\mathcal{X}$, and then classify a given observation to the class with highest predicted probability [62]. Given two observations $\boldsymbol{x}_{1}$ and $\boldsymbol{x}_{2}$ in the training dataset $\mathcal{X}$, the distance between two can be defined as $D\left(\boldsymbol{x}_{1}, \boldsymbol{x}_{2}\right)$. A popular way of viewing this through the use of the Euclidean distance method. This is depicted as:

$$
D\left(\boldsymbol{x}_{1}, \boldsymbol{x}_{2}\right)=\sqrt{\sum_{i=1}^{d}\left(x_{1}^{(i)}-x_{2}^{(i)}\right)^{2}}
$$


Measuring the Quality of Fit Once data is trained using a learning algorithm, the performance of the algorithm must be evaluated to determine how well the predictions match the observed data. For regression based algorithms, the most commonly used measure is the mean square error (MSE). This model is presented as:

$$
M S E=\frac{1}{n} \sum_{i=1}^{n}\left(y_{i}-\hat{f}\left(x_{i}\right)\right)^{2}
$$

where $\hat{f}\left(x_{i}\right.$ is the prediction that $\hat{f}$ gives for the ith observation and $\dagger_{i}$ is the corresponding true value.

Decision Trees Decision trees (DT) are known as simple analytical tools for regression and classification that can interpret qualitative and quantitative predictors then display them graphically. The graph typically uses branches to illustrate possible outcomes of decisions. DT works by organizing the data into two or more homogeneous regions based on the most significant splitter among the independent variables [59]. One disadvantage of trees is that compared to other regression and classification approaches, they do not have the same level of predictive accuracy. This predictive performance can be improved through the use of bagging and random forests.

Random Forests Random forests includes generating various trees which are then integrated to produce a single agreeable prediction. In the random forests, the variance of the model is reduced by way of segmenting a sample of features unlike splitting at all of the features as in DT [59].

\subsubsection{Unsupervised Learning}

The unsupervised learning of ML is quite different than that of supervised learning. This method uses hidden patterns to produce deductions from unlabeled data. This type of 
technique is more ideal in cases where the information from the data is unclear or there is no specific goal for the user. The application areas contain data clustering, dimensionality reduction, and association rule learning. Among the many unsupervised ML models, clustering is the most commonly used. Its application can extend from the areas of object recognition to gene sequencing.

\subsection{Summary}

Given all the sensors mentioned in this chapter, there exists an urgent need for incorporating them with IoT and Artificial Intelligence. This would allow real-time monitoring and control of farm systems, thus making it easier for farmers to optimize production and minimize resource utilization. The literature identifies that due to the excessive utilization of fertilizers and pollution effects on the environment, a growing concern of pollution has warranted the need for technologies that can better monitor soil nutrients and their fate. The conventional laboratory methods may offer highly accurate analysis of soil chemistry, in-situ based soil nutrient sensors that offer real-time feedback are needed in order to truly increase the efficiency of farming and managing the environment. As compared to conventional lab instruments for soil nutrient analysis, it was found that ins-situ based sensors are more advantageous due to their low-cost, and highdensity measurement capability for large-area soil nutrient mapping. Although these sensors are becoming less expensive to manufacture and can provide comparable results to laboratory soil analysis, there still exists a need to understand the effects of soil heterogeneity on the response of both optical and electrochemical sensors. More durable and accurate sensor systems that consider the effects of soils heterogeneity and offer soil specific calibrations are needed to move towards commercializing these platforms. When interfaced with long-range low powered radio modules, these sensor platforms are 
able to implement real-time prediction, control, and decision management for large scale precision agricultural practices. Coupling these real-time sensor networks with machine learning further enhances the autonomous management of farms soils. ML and big data from in-situ sensors allows farmers to harness the predictive powers of algorithms in order to optimize production and minimize resources far greater than technologies of the green revolution. As the saying goes, the revolution will be live! In the case of agriculture, the revolution will be real-time. 


\section{CHAPTER 4}

\section{IMPLEMENTING PRINTING: A LOW-COST LARGE VOLUME MANUFACTURING TECHNOLOGY}

\subsection{Overview}

This chapter introduces low-cost large volume manufacturing technologies for producing soil nutrient sensors. Such technologies include roll-to-roll printing, ink-jet printing and laminating. Section 4.2 introduces printing and identifies market shares and compound annual growth rate of printed sensors. Section 4.21 discusses types of printing which include flexographic, gravure, screen printing, ink-jet printing. Encapsulation techniques for protecting circuitry are also discussed in this section. In section 4.3 challenges of printed electronics are discussed.

\subsection{Printed Technologies}

Printed technologies have become exceedingly popular in the manufacturing sector over the past years [63]. In year 2027 alone printed sensors are expected to reach $\$ 7.6$ billion with printed technologies and sensors extending from solar cells, humidity sensors, temperature sensors, photo detectors and electrochemical sensors. The figure 4.1 shows an example of a relative market size of printed sensors by year 2024. Further, the economic impact of these sensors in terms of compound annual growth rate within the next 10 years is shown in the figure. These printed sensors show great promise for future as they are also simple to fabricate. Table 4.1 reveals a list of typical methods to fabricate sensors.

Printed technologies have been found perfect for large scale manufacturing given it produces less waste by utilizing fewer material and is capable of producing large batches 


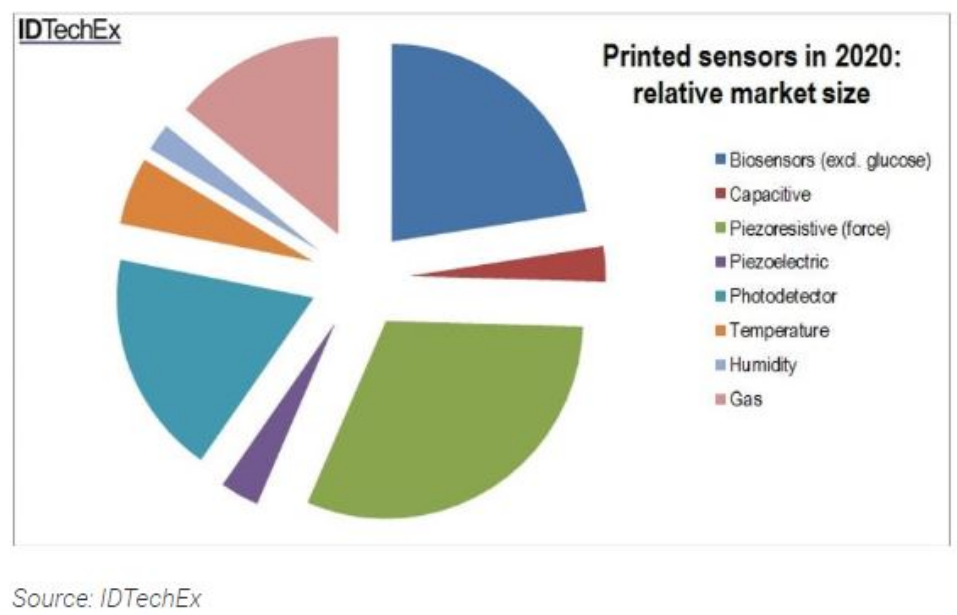

Figure 4.1: Illustration of a pie chart which details the relative market share of printed sensors in 2025. [64]

Table 4.1: Typical methods for fabricating sensors which include the silicon wafer process, roll-to-roll printing and ink-jet printing. The typical electrode thickness, resolution, printing speed that can be achieved for each method is also listed in addition to cost per meter and image carrier type.

\begin{tabular}{c|c|c|c}
\hline & Si Process & Roll-to-roll & Ink-jet Printing \\
\hline Resolution & nm scale & $15-30 \mu \mathrm{m}$ & $15 \mu \mathrm{m}$ \\
Electrode film thickness & $1 \mathrm{~nm}$ to $+100 \mu \mathrm{m}$ & $0.6-30 \mu \mathrm{m}$ & $0.01-0.5 \mu \mathrm{m}$ \\
Printing Speed (m/min) & $<1$ & $0.6-180$ & $0.02-5$ \\
Image Carrier & Mask & Plate/Stencil/Gravure & Virtual PC \\
Cost $(\$ / \mathrm{m})$ & $>100$ & $<100$ & $-\$ 50$ \\
\hline
\end{tabular}

by printing on large areas [65] [66] [67]. Unlike nano-fabrication processes and silicon technologies, printed technologies do not require photo-lithography, high vacuum and temperature sensitive deposition processes, instead, its processing can be done in ambient conditions [68]. In addition to all the above advantages, printed technologies are a perfect candidate for producing low-cost products due to the fact that it is highly efficient when utilizing resources during the fabrication process. 


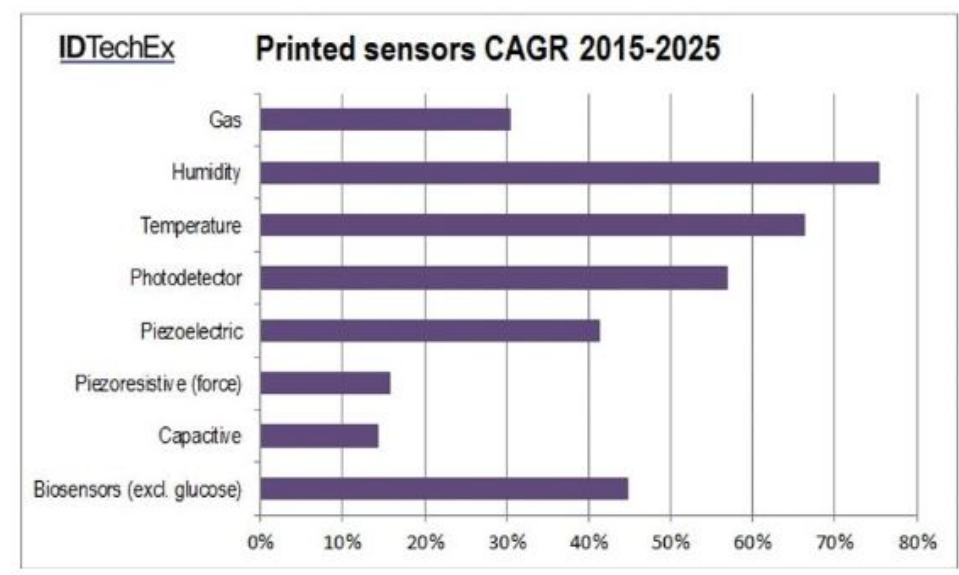

Source: IDTechEx

Figure 4.2: Illustration of a bar graph detailing the compound annual growth rate for printed sensors between years 2015-2025. [69]

\subsubsection{Types of Printing}

Printed technologies utilize high throughput techniques to produce electronic sensors devices and circuit [70]. This is usually accomplished by roll-to-roll (gravure, flexography, screen printing) and ink-jet printing. Roll-to-roll techniques have been considered as contact methods which requires some type of design transfer from a solid carrier onto a separate material. To achieve the printed circuits and sensors, the use of special conductive, semi-conductive and dielectric inks are required. The roll-to-roll method excludes the ink-jet printing method which will be further discussed in a proceeding subsection.

\section{Flexographic Printing}

Flexographic printing, also known as flexoprinting, is considered an indirect printing process which is capable of depositing many different thicknesses with similar resolution on a wide variety of substrates [71]. Much literature has reported the use of this technique, including [72] [73] [74] [75]. As shown in the figure 4.3, the flexographic 


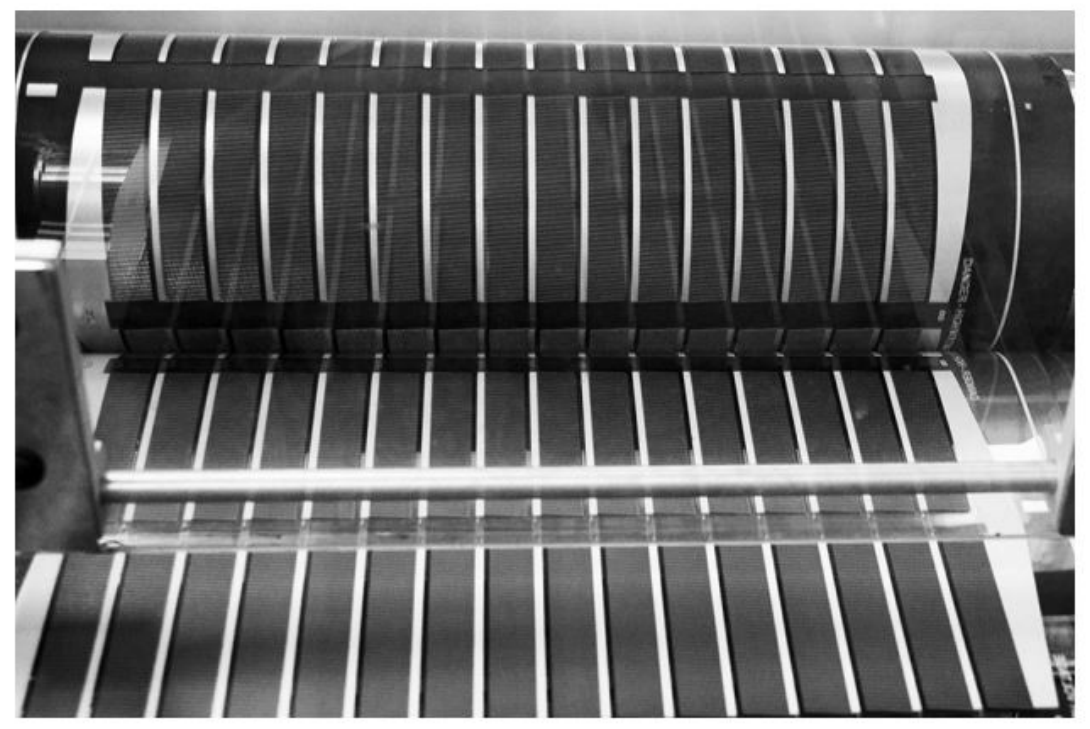

Figure 4.3: Illustration of flexoprinting. Reprinted from [70] with permission from Elsevier.

printing technique encompasses the use of an impression cylinder, plate, anilox roller, doctor blade, and an inking unit. In this method, the transfer of ink is made between a soft plate cylinder, known as the flexo plate, and the substrate. This ink transfer process is similar to that of a stamp, as the soft cylinder material is usually made of rubber or some type of photo-polymer.

\section{Gravure Printing}

Gravure printing is widely used for large volume printing and packaging, and is popular due to its high printing speed and exceptional printing quality. Much work for fabricting electrodes has been accomplished using this method, including [76] [77] [78]. As shown in the figure 4.4, a metal coated gravure cylinder, steel doctor blade, rubber impression roller and ink bath are the main components of a typical gravure printing process. In the gravure printing method the ink is passed on from carved microcavities embedded into the printing cylinder to form the printing pattern. A secondary impression cylin- 


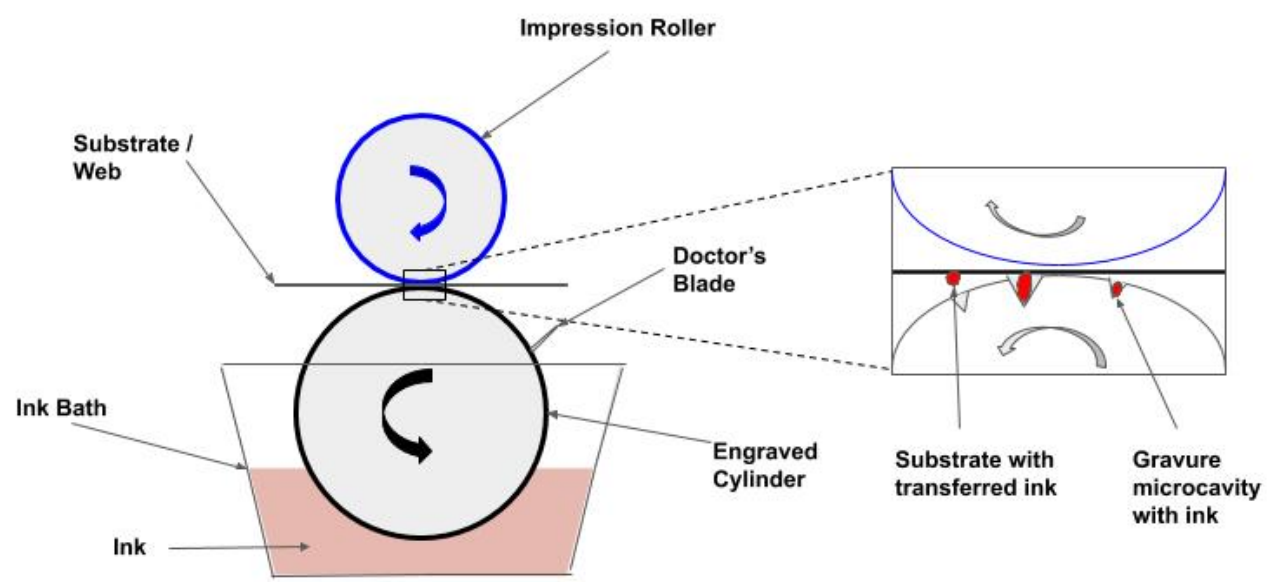

Figure 4.4: Illustration of a typical gravure printing process which includes the components.

der is used to push the web and substrate against the primary printing cylinder and the ink is transferred from the cavities to the web. The printing cylinder, which also is partially submerged in a volume of ink, utilizes a doctor blade to remove excess ink from the cylinder so that only the micro cavities contain ink. Low viscous inks can be used in this process and in many cases produce with printing speeds as high as $15 \mathrm{~m} / \mathrm{s}$ [70].

\section{Screen Printing}

Screen printing is accomplished by utilizing a contact technique to transfer an image pattern to a substrate. Much work has been reported using this type of printing, including electrode printing for various applications [79] [80] [81] [82] [83] [84] [85]. The materials used for screen printing can vary depending on solvents and cleaning agents but all require the use of two components; a rubber squeegee and a screen printing plate. 
The screen printing plate consists of an aluminum / steel frame, a mesh screen fabric, and a stencil. In this technique, some force from the squeegee is utilized to push a highly viscous ink with thixotropic properties through a solid mesh. The transferred image pattern which passes through the mesh is determined by the pore space of the mesh and the viscosity of the ink and usually transfers an ink pattern with a thickness between 10-500 microns in height. Currently there are two existing methods for screen printing: flatbed screen printing and rotary screen printing. The flatbed process is a three step process that involves first, the contact of the mesh with the stencil, then second, the sweeping of the squeegee across the screen to force the ink through mesh and transfer the desired pattern. Finally, the screen and stencil are removed from substrate and the steps can be repeated. This method has been suitable for large-scale manufacturing as it allows large scale printing of areas up to 10 square meters per pass and allow speeds up to $35 \mathrm{~m} / \mathrm{min}$. It also a common method for small scale projects and lab experiments. The rotary screen printing method can be thought of as a continuous pattern printing process. The process utilizes ink and a stationary squeegees built inside the cylinder shape screen which forces ink through the screen as it rotates. This roll-to-roll printing method can allow prints up to $100 \mathrm{~m} / \mathrm{min}$ making it ideal for large-scale manufacturing.

\section{Ink-Jet Printing}

As stated previously, ink-jet printing differs from the traditional contact printing techniques as it lacks the need for a physical mask or image carrier. Much has been reportde on the use of this type of printing for device fabrication [86] [87] [88] [89]. Two dimensional thin films and 3D patterns can be printed by using direct deposition from a digital file containing a pixelated drawing. The two most common types of ink-jet printing are the drop-on-demand and continuous methods [70]. In continuous method, a continuous stream of droplets is formed, then, a voltage source electrostatically controls the deflec- 


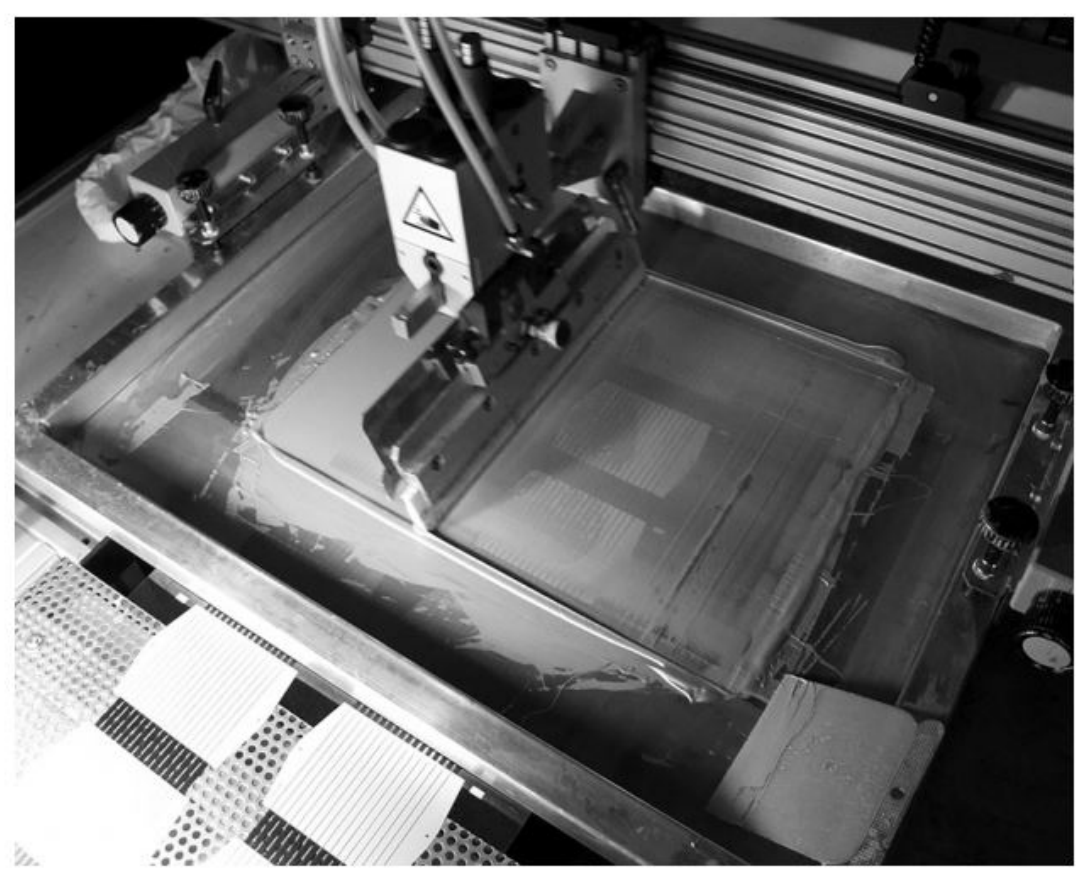

Figure 4.5: Illustration of a flat bed screen printing process of silver top electrodes. Reproduced from [70] with permission from Elsevier.

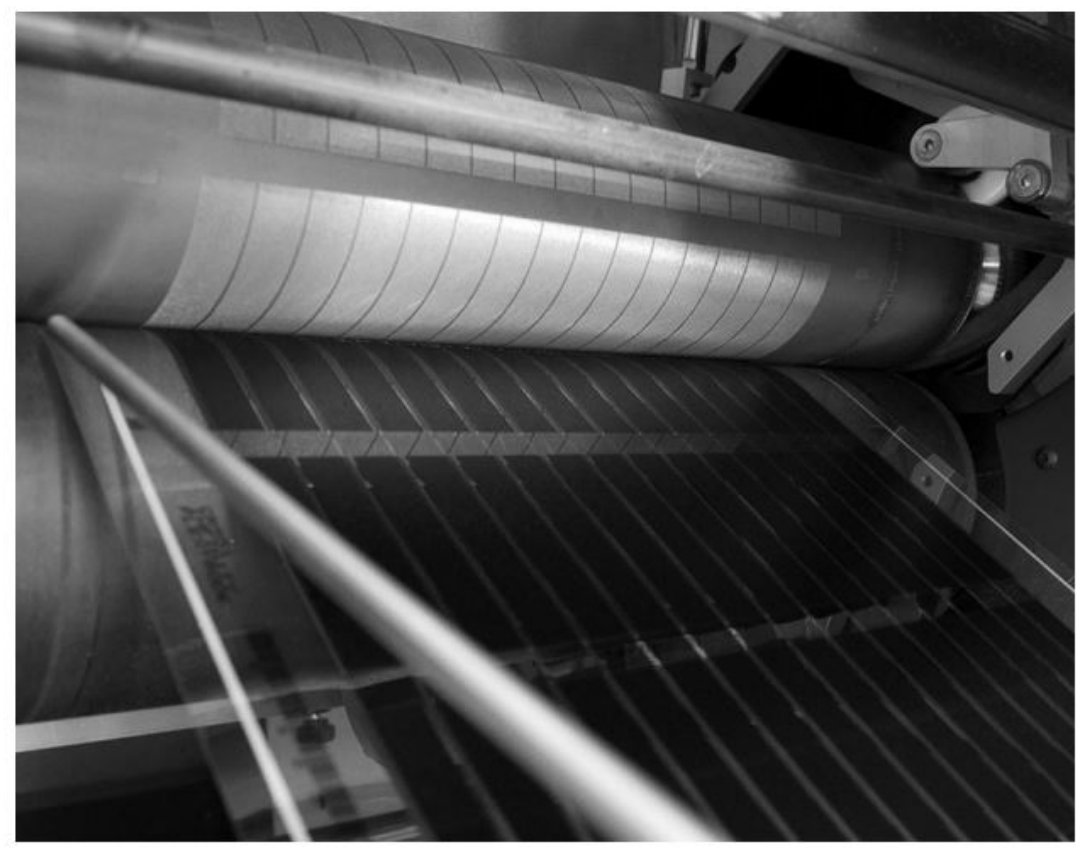

Figure 4.6: Illustration of a rotary screen printing of PEDOT. Reproduced from [70] with permission from Elsevier. 


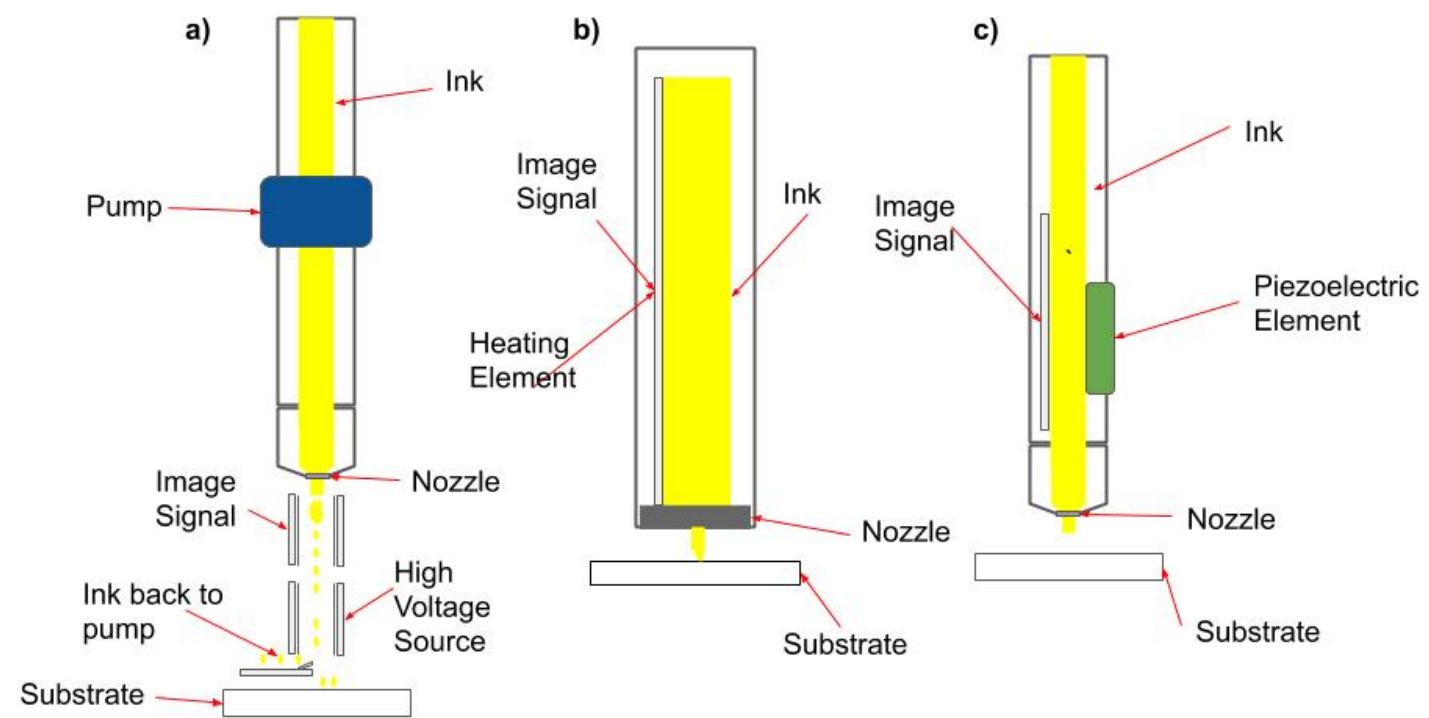

Figure 4.7: Illustration of ink-jet printing techniques. This includes the a)continuous drop technique, b)thermal technique, and c) the piezoelectric ink-jet printing technique.

tion of ink to print desired patterns on a substrate. The non-deflected ink is routed back into the ink cartridge. The drop on demand method can be further categorized into three types; piezoelectric ink-jet printing, thermal ink-jet printing, and electrostatic ink-jet printing [90]. A piezoelectric device is positioned on top of the nozzle within the ink channel. By applying a voltage to the piezoelectric device, the device can then enlarge to facilitate the release of ink droplets through the nozzle. Thermal ink-jet printers heat the ink inside the nozzle to expel the ink droplet from the nozzle. Electrostatic ink-jet printers require that the ink be charged and utilizes an electric field between the nozzle and an electrode, attracting free charges within the ink to the surface. Ink-jet printing is a common method as it allows printing on various materials. This type of printing hoever, requires special inks for printing conductive electrode [91]. Most commercial ink-jet printers can allow high resolutions and print with speeds up to $75 \mathrm{~m} / \mathrm{min}$ [70]. 


\section{Encapsulation techniques}

Encapsulation techniques are needed to protect the device from the surrounding environment, allow little to no permeability of oxygen and water, and protect from mechanical stress. Lamination has been considered one of the most popular methods of encapsulation, which consists of joining sheets. This is usually on both sides through a layering process [70].

Cold Lamination Cold lamination is accomplished by using a pressure sensitive adhesive and liner to laminate a surface. The liner is used to protect the adhesive quality of the laminate and is peeled off before applying the adhesive to a substrate. As shown in the figure 4.9, large manufacturing machine utilize two cylindrical rollers to press together the adhesive and substrates to ensure good adhesion during the lamination process. The lamination process can facilitate adhesive layering of 50 microns and greater and can process up to $20 \mathrm{~m} / \mathrm{min}[70]$.

Hot Lamination Hot lamination technique functions by way of a heat activated laminate which is joined to the substrate when pressed between two heated elements. The adhesive melts as it is heated and forms a tight seal once it begins to cool. This process can allow thinner layers down to 20 microns and produce large batches per minute which makes it suitable for large-scale manufacturing.

UV Lamination UV lamination functions by utilizing an adhesive that is UV sensitive. The UV adhesive is added to the laminate then joined to the substrate by exposing the laminate and substrate to UV light. 


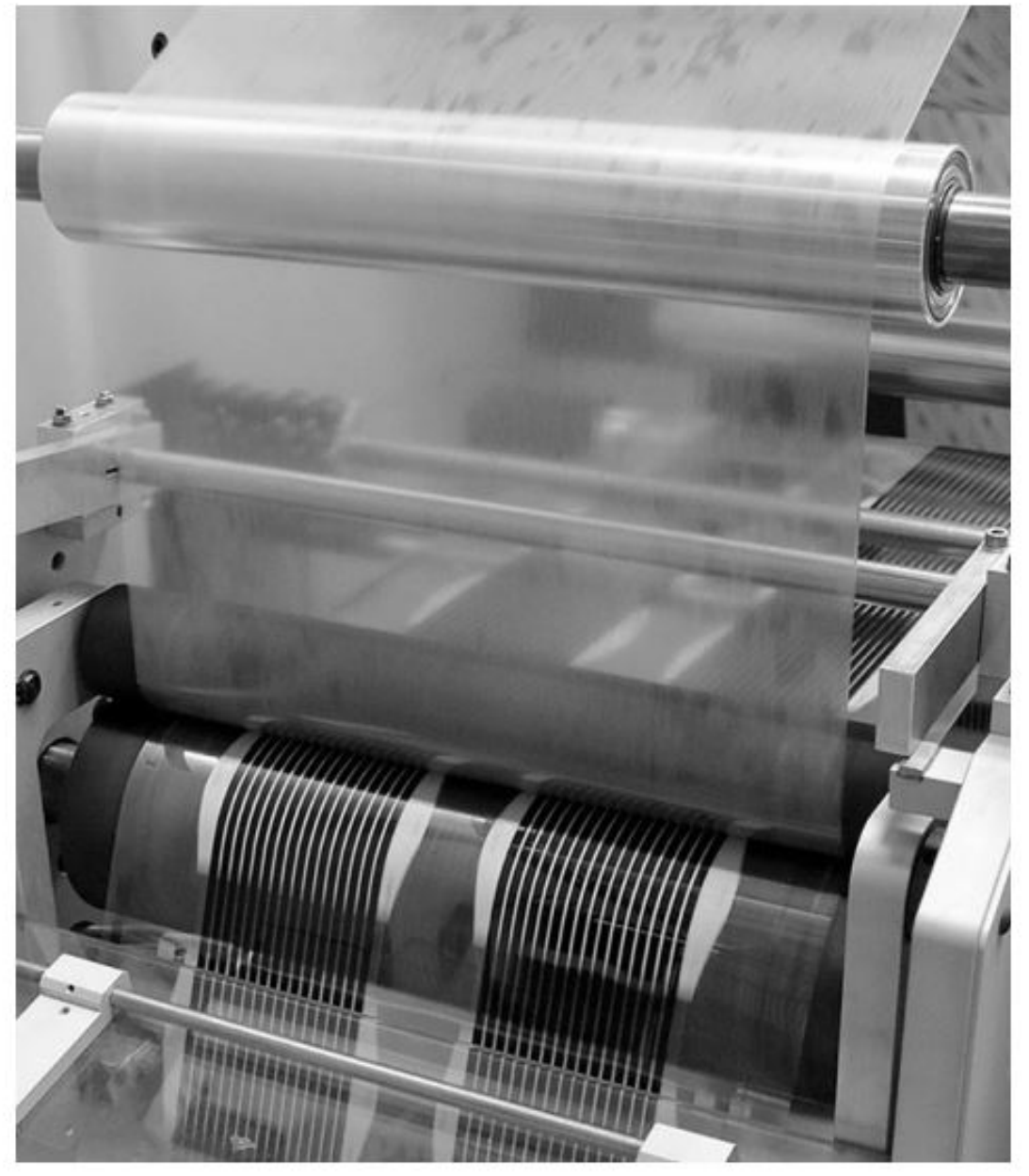

Figure 4.8: Illustration of hot lamination process. Reproduced from [70] with permission from Elsevier. 
a)

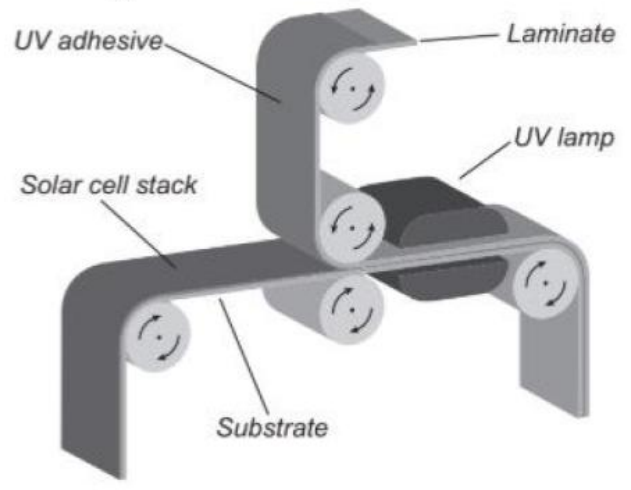

b)

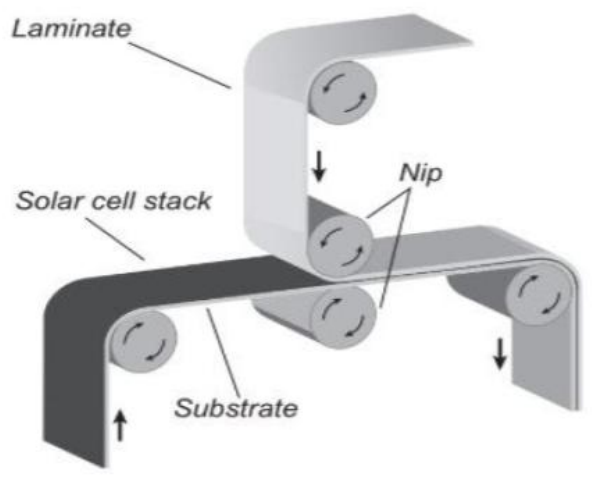

Figure 4.9: Illustration of lamination techniques. a) UV lamination where the UV reactive adhesive is applied to via printing technique and b) cold and hot lamination technique. Here the adhesive is applied to the laminate via print method and nip cylinders provide heat to cure the adhesive for hot lamination. [70]

\subsection{Printed Electronics and Challenges}

Many challenges may arise with printed electronics due to substrate and stencil shifting when transferring an image to a substrate, therefore alignment and machine stability must be carefully examined. Flat bed printing also presents challenges when the ink remains in ambient conditions. These challenges include solvent evaporation, which highly influences the printing process. Cleaning and maintenance of roll-to-roll printing machines have been reported to be a challenge. The rotary screen may offer better definition than flatbed printing however the screen is significantly more expensive and more difficult to clean due to limited access within the cylindrical roller. Though ink-jet printing is quick, the printing area is limited. In addition, special inks are required for thermal ink-jet and electrostatic ink-jet which may be either expensive or difficult to obtain. [90] reported that major drawbacks with ink-jet printing stems from the ink properties (density, surface tension, viscosity, boiling point, etc.). These ink properties must 
modified to meet various specifications such as nozzle size, printing surface and materials. Such Specifications make it more difficult for ink selection. Further challenges lie in the lamination of printed devices. The stability is usually compromised with hot and cold lamination techniques, as bleaching near the edges of the active layers are observed, especially with single laminate layers. The UV lamination technique is by far the more complex, as it requires an extra step to deposit the UV adhesive onto the laminate [70].

\subsection{Summary}

In summary, printing is a large scale manufacturing technique that can be adopted for the production of low-cost senors for precision agriculture. These techniques include roll-to-roll methods and ink-jet printing. The greatest advantage of these methods over traditional clean-room fabrication techniques is the ability to produce large batches of sensors and devices in such a short time using inexpensive materials. Unlike clean-room facilities, which requires vacuum chambers and low particulate spaces for electrode de-

position, fabrication of sensors and electrodes via printing method can be accomplished in ambient conditions virtually in any space. 


\section{CHAPTER 5}

\section{UNDERSTANDING THE ROLE OF SOIL HETEROGENEITY ON NUTRIENT SENSOR ACCURACY AND DRIFT}

\subsection{Overview}

Disposable large scale sensing for soil nutrients in real-time has not been possible because of accuracy and drift. In this chapter, mass soil water content $(\theta \mathrm{m})$, organic matter $(\mathrm{OM})$, particle size (Ps), electrode placement in soil $\left(\mathrm{E}_{\text {place }}\right)$, time after applied moisture (TAM)) and lead length (LL), were selected to investigate their effect on the analytical performance of a polymer based soil nitrate sensor system, fabricated in house. Sensor readings were significantly $(\mathrm{p}<0.05)$ affected by the $\theta \mathrm{m}$. Section 5.3 presents the materials and methods used in the experimental sections of this chapter. In section 5.4 the results and discussion are presented, followed by the summary in section 5.7. Overall, this chapter explores and highlights the need to account for the effect of each predictor on large-scale in situ nutrient sensor measurements in order to improve sensor accuracy and reduce drift.

\subsection{Introduction}

Nitrogen $(\mathrm{N})$, is the main macronutrient for plant growth and is often exploited in the form of nitrate (NO3-) fertilizer to optimize crop growth and increase yields. However, excess amounts of NO3- in soils are subject to runoff and leaching into surface waters, ground waters, and drinking water, negatively impacting the biodiversity of the ecosystem and poses hazard to human health [92]. In many instances, $\mathrm{NO}_{3}^{-}$has been found to exceed the $10 \mathrm{ppm}$ Maximum Contaminant Level (MCL) in drinking water set by the Environmental Protection Agency (EPA) [93]. Due to these events and concerns, 
much attention has grown towards precision sensing devices for agricultural and environmental monitoring, especially for understanding those biochemical behaviors of soils [94]. For farmers, precision agriculture necessitates real-time information on condition of soils, crops, pests, moisture and yield for optimizing production and minimizing resource utilization. In the case of soil nutrient sensing, researchers are working to produce nitrate sensors which can be deployed sub-soil and to continuously collect accurate soil nitrate data over long periods of time [95]. However, to our knowledge there are currently no electrochemical based sensing apparatus such as this which is popular or commercially available. Instead, the most conventional methods for determining soil nutrient concentration are done by 1) analyzing soil core extracts using bulky and expensive laboratory instruments, and 2) flow injection analysis (FIA) systems interfaced with expensive farm vehicles [23].

Cost friendly alternatives for soil nutrient analysis using ion selective electrodes (ISEs) have gained considerable attention over the years. Conventional membrane ISEs employ the use of ionophores to detect target analytes, and have their development have been explored in [96] [97] [98]. However, due to the limitations imposed by traditional ionophore ISEs, they are being replaced with conductive polymer electrodes such as molecular imprinted polymers (MIPs) because they are more durable cost efficient, do not require additional fill solutions, and can be miniaturized and fabricated with ease [99] [100] [101].

ISE's for nitrate using pyrrole and a nitrate electrolyte have been studied [101] [102] [55] [103], and demonstrated large linear ranges, better selectivity and sensitivity than conventional electrodes, and less sensitivity to changing $\mathrm{pH}$ conditions [101]. The pyrrole monomer, when polymerized, becomes polypyrrole, and is widely studied due to its high conductivity and ease of use [55]. [37] developed an IoT nitrate sensing apparatus for soil leachate, and was also based on polypyrrole (PPy) membranes for in situ mea- 
surements. However, [37] did not address the challenges imposed by soil physical and chemical behaviors which may alter sensor output.

According to literature, soil sensor performance can be influenced by the effects of most soil physical and chemical parameters [104], there is little or almost no documents on the effects of soil moisture content $\left(\theta_{m}\right)$ on $\mathrm{NO}_{3}^{-}$based PPy sensor performance. The dynamic moisture heterogeneity of soils, even over short distances presents various changes in soil resistivity, thereby influencing the sensor-to-soil interface and sensor output. Here, this chapter addressed this fundamental challenge with in situ soil nutrient measurements. Understanding the effect of $\theta_{m}$, and LL, on polymer based nitrate sensor analytical performance helps efforts in developing an approach for correcting in situ soil nutrient readings at different moisture contents and distance scales.

\subsection{Materials and Methods}

Reagents Deionized water of $17.8 \mathrm{~m} \omega$ was used to prepare solutions and hydrate soils to various moisture contents. Pyrrole monomer and sodium nitrate were acquired from Sigma-Aldrich and were analytically pure grade. The pyrrole is photosensitive, therefore was refrigerated and stored in dark containers. Before each use, the monomer was also purified by purging with nitrogen gas for 15 minutes to remove oxygen.

Electrode preparation Electrode preparation was carried out similarly to that in [37]. The polymerization of pyrrole doped nitrate onto inkjet printed carbon silver nanoparticle electrodes were performed electrochemically using a potentiostat/galvanostat $(\mathrm{CH}$ Instruments, Model 720A). The polymerization recipe included $1 \mathrm{M}$ pyrrole and $0.5 \mathrm{M}$ of $\mathrm{NaNO}_{3}^{-}$electrolyte solution which was purged for 15 minutes before polymerization. 
During the polymerization process, the PGSTAT applied a constant current source between $100 \mu \mathrm{A}$ and $1.5 \mathrm{~mA}$ for 900 seconds.

Preparation of sandy soil for nitrate sensor response Nitrogen fertilizer by Potash Corp and Quikrete all-purpose sand was acquired from Home Depot in Miami, FL. The Potash Corp is $100 \%$ Nitrogen based fertilizer, which contains an N-P-K ratio of 46-0-0. The $\mathrm{N}$ based fertilizer consists of large particle granules and were grinded using conventional glass bowl to a desired particle size prior to mixing in sandy soil. The graded coarse sand meets ASTM C 33 specifications and is 100\% silica quartz by weight. Quartz sand has been purposely chosen due of the absence of impurities, organic matter or salinity, does not shrink and swell like clay texture, and because the cation exchange capacity of quartz sand is relatively low and will not interfere with the treatment effect of the experiment. The sand was sieved to particle sizes of $710 \mu \mathrm{m}$ and $2 \mathrm{~mm}$ and washed thoroughly to remove any additional impurities. Also, at this point baseline parameters were recorded including sandy soil nitrate levels and pH. Sandy soil was then oven dried at 60 degrees $\mathrm{C}$ for a period of 24 hours. After dehydration, the percent of soil water was derived using gravimetric water content calculation, equation:

$$
\theta_{m}=\frac{m_{\text {water }}}{m_{\text {soil }}}=\frac{m_{\text {wet }}-m_{d r y}}{m_{d r y}} \times 100 \%
$$

where gravimetric percent water content, $\mathrm{m}$, is equal to the mass of the water divided by the mass of the soil multiplied by 100 percent.

Dried sandy soils were spiked with LOW, MED, and HIGH concentrations of urea nitrogen fertilizers, then thoroughly mixed. The spiked sandy soils were then packed in $50 \mathrm{~mL}$ tubes which also housed the nutrient selective electrodes. Water content levels $(0 \%, 4.54 \%, 9.09 \%, 13.63 \%, 18.18 \%, 22.72 \%$, and $27.27 \%)$ were unique for each sand tube and were manipulated by uniformly adding volumes of deionized water in addition to the fertilizer treated and oven-dried sandy soils. Sensor readings were acquired using 
multiplexed nutrient sensor array built in house at FIU EC, as shown in figure 1 The experimental design was a factorial design with moisture content level, sand particle size and nitrate concentration as treatment variables.

Setup for multiplexed sensing of nitrate in sandy soil The N-doped PPy soil nitrate sensing platform employs a multiplexed sensor array to an oxidation/reduction potential (ORP) circuit and can communicate via LoRa technologies. The multiplexed sensor array allows us to easily select between soil sensors from each treatment group. Sensors were arranged in a matrix to measure multiple samples containing different moisture and fertilizer levels. Reed relays were used to control the rows and columns of the matrix acting as a multiplexer, allowing you to read a specific sensor from a common connection.

Amperometric Measurements Amperometric measurements were taken using CH Instruments Model 600E series potentiostat/galvanostat electrochemical analyzer/workstation (Austin, TX). The measurements were taken using standard three electrode configuration with N-doped PPy working electrodes, carbon reference and counter electrode.

Machine Learning The over all goal of this experiment was to test the effect of predictor values on the in-situ based sensor readings (SR). The target value of this model was SR, while the predictor values were $\theta_{m}, \mathrm{OM}, \mathrm{P}_{\text {size }}, \mathrm{E}_{\text {place }}$, and TAM (table 5.1). The data from the predictors were extracted from the experimental set-up of in-situ based nutrient sensors buried in quartz sand. Much of the predictors were categorical, therefore one-hot encoding with pandas was done to ensure the learning algorithms recognized the input variables. Since many variables were also on different scale, they were also normalized. The model was built on a training set consisting of 70 percent and the re- 

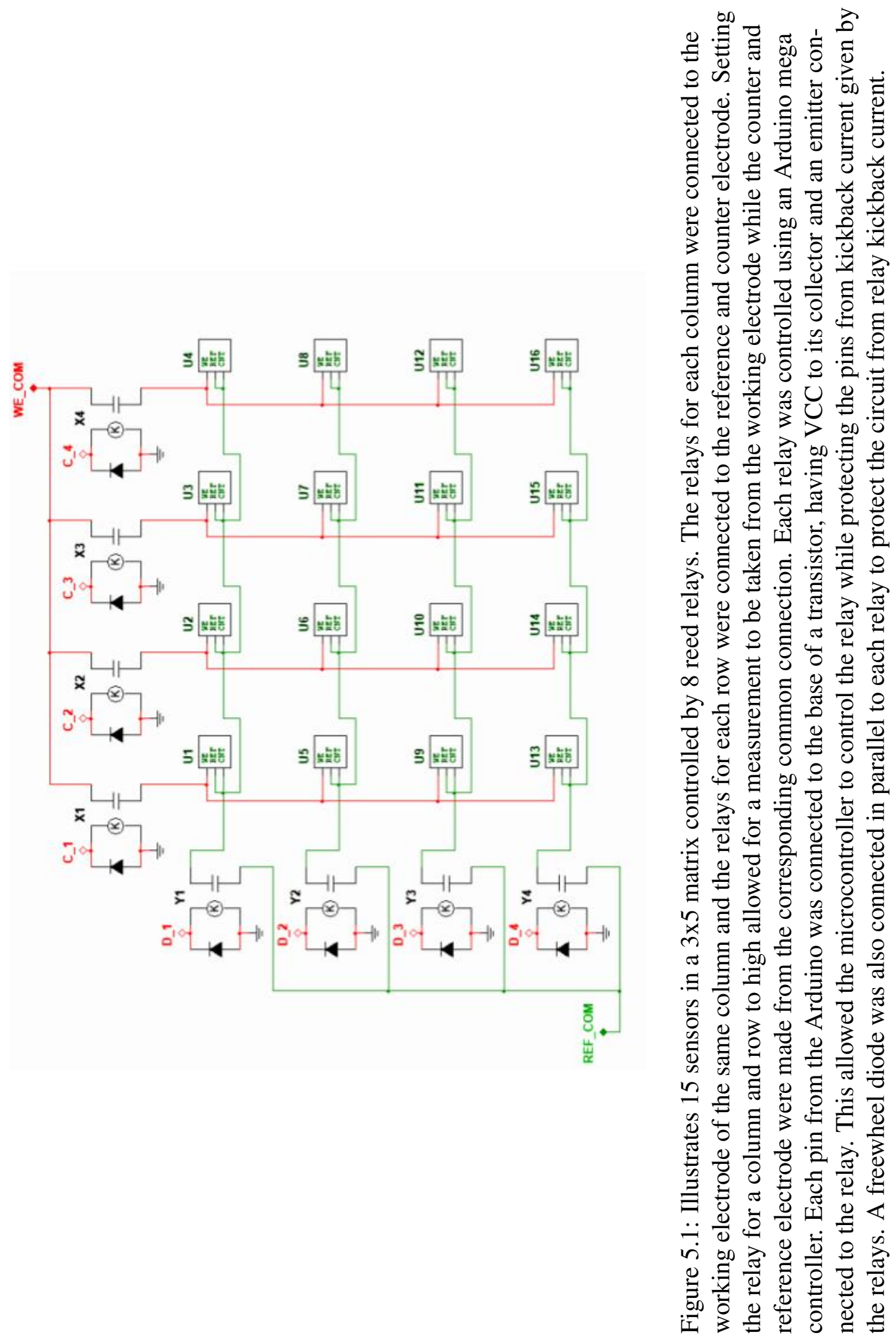
Table 5.1: Predictor attributes in SR model

\begin{tabular}{|c|c|c|}
\hline $\begin{array}{l}\text { Predictor } \\
\text { code }\end{array}$ & Predictor name & Predictor description \\
\hline$\theta_{m}$ & Soil moisture content (\%) & $\begin{array}{l}\text { The amount of moisture content } \\
\text { present in the soil }\end{array}$ \\
\hline $\mathrm{OM}$ & Organic Matter (\%) & $\begin{array}{l}\text { The amount of organic matter con- } \\
\text { tent present in soil }\end{array}$ \\
\hline $\mathrm{P}_{\text {size }}$ & Soil particle size $(\mu \mathrm{m}$ and $\mathrm{mm})$ & Soil granular size \\
\hline $\mathrm{E}_{\text {place }}$ & Electrode placement ( $\mathrm{mm})$ & $\begin{array}{l}\text { The placement of electrode in three } \\
\text { depths }\end{array}$ \\
\hline TAM & Time after applied moisture (seconds) & $\begin{array}{l}\text { Measurement taken after moisture } \\
\text { is applied to sandy soil }\end{array}$ \\
\hline
\end{tabular}

maining 30 percent consisted the test set where the performance was tested randomly.

\subsection{Results and Discussion}

Calibration of N-doped PPy Soil Sensors Prior to moisture effect studies, the response of the N-doped PPy electrodes were tested for nitrate ions in calibration solutions. Figure 5.2 illustrates a calibration plot of the N-doped PPy sensors created using the peak currents at each molar concentration. A linear range of $1 \mu \mathrm{M}-60 \mu \mathrm{M}$ was observed. The linear fit of the form is:

$$
I(A)=2.97^{-05} \text { conc. } M+1.59 E^{-04}
$$

From the slope of the calibration plot, the sensitivity was derived as $2.976 \mathrm{E}^{-05}$ and a linearity coefficient of 0.92 . The sensor also exhibited a response time which compared favorably to other doped PPy electrodes (CITE). 


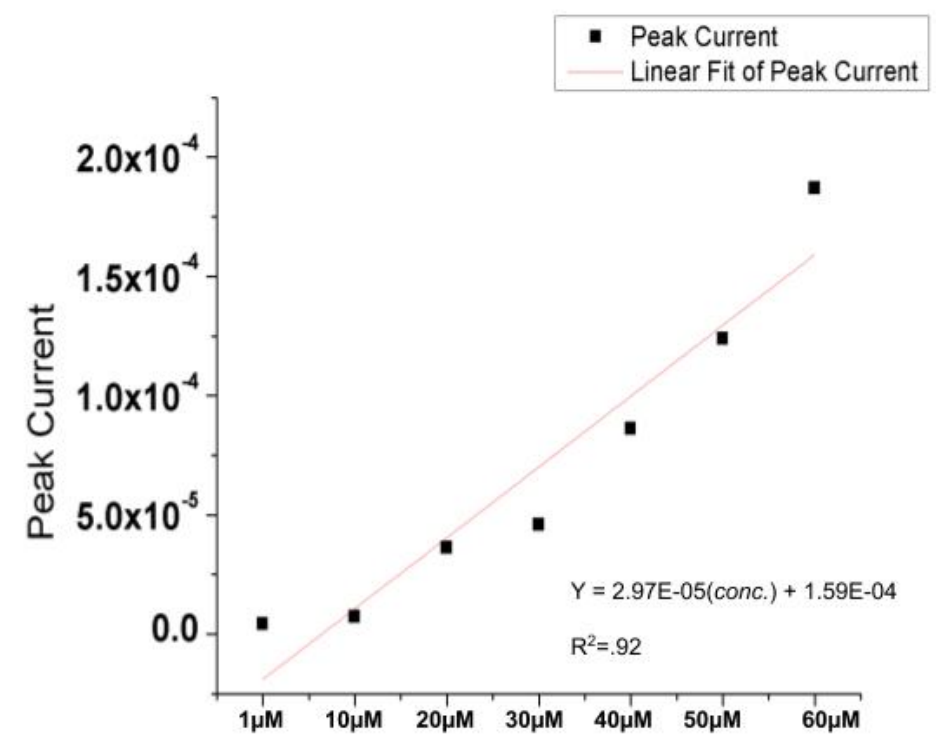

Figure 5.2: Calibration plot for N-doped PPy sensor; peak current vs nitrate concentration $(1 \mu \mathrm{Mol}$ to $60 \mu \mathrm{Mol})$ 


\subsubsection{Infiltration and Percolation Measurements}

Infiltration and percolation measurements were performed to understand the transport of solutes in sandy soil. The experimental set-up consisted of a $5 \mathrm{~mL}$ vial and a $50 \mathrm{~mL}$ vial filled with quartz sand and a set of electrode pins pierced through the sides of the vials at three different depths to facilitate the measurement of flow trough sandy soil. Flow in unsaturated soil can also quantified as effective saturation $\left(\mathrm{S}_{e}\right)$ can be mathematically expressed by relating the residual moisture content to saturation:

$$
S_{e}=\frac{\theta-\theta_{r}}{n-\theta_{r}}
$$

where, $\left(\mathrm{S}_{e}\right)$ is the effective saturation, $\theta_{r}$ is the residual moisture content and $\mathrm{n}$, is saturation.

A conductive solution was prepared then applied to the surface of the sand vials to mimic irrigation events. The electrodes measured electric conductivity changes as the solute moved through soil. Measurements were found to agree with prior simulations of saturation vs time, which were done using COMSOL Multiphysics.

The percolation experiments aided in determining the optimal time to take a measurement after moisture was applied (TAM), considering both percent of moisture applied and depth of sensor. As shown in figure 5.3, $18 \%$ moisture by volume was added to the sand column in one disbursement and the current changes in 3 zones were observed over a span of 2500 seconds. Zone 1 , as denoted by e 1 in the figure 5.3, displays initial infiltration (inside green circle) at TAM 1. Then, the current at e1 began a steady decline for the remainder of the experiment. As the current in e 1 declines, e 2 current began to increase near 100 seconds. This indicated percolation through zone 2. At approximately 1250 seconds (TAM2), e2 had reached its current peak, at this time, a sharp current incline was observed in zone 3 (e3). Near 2300 seconds, the current at e1 and e2 had begin to plateau, while e3 continued to a peak at 2500 seconds (TAM3). 


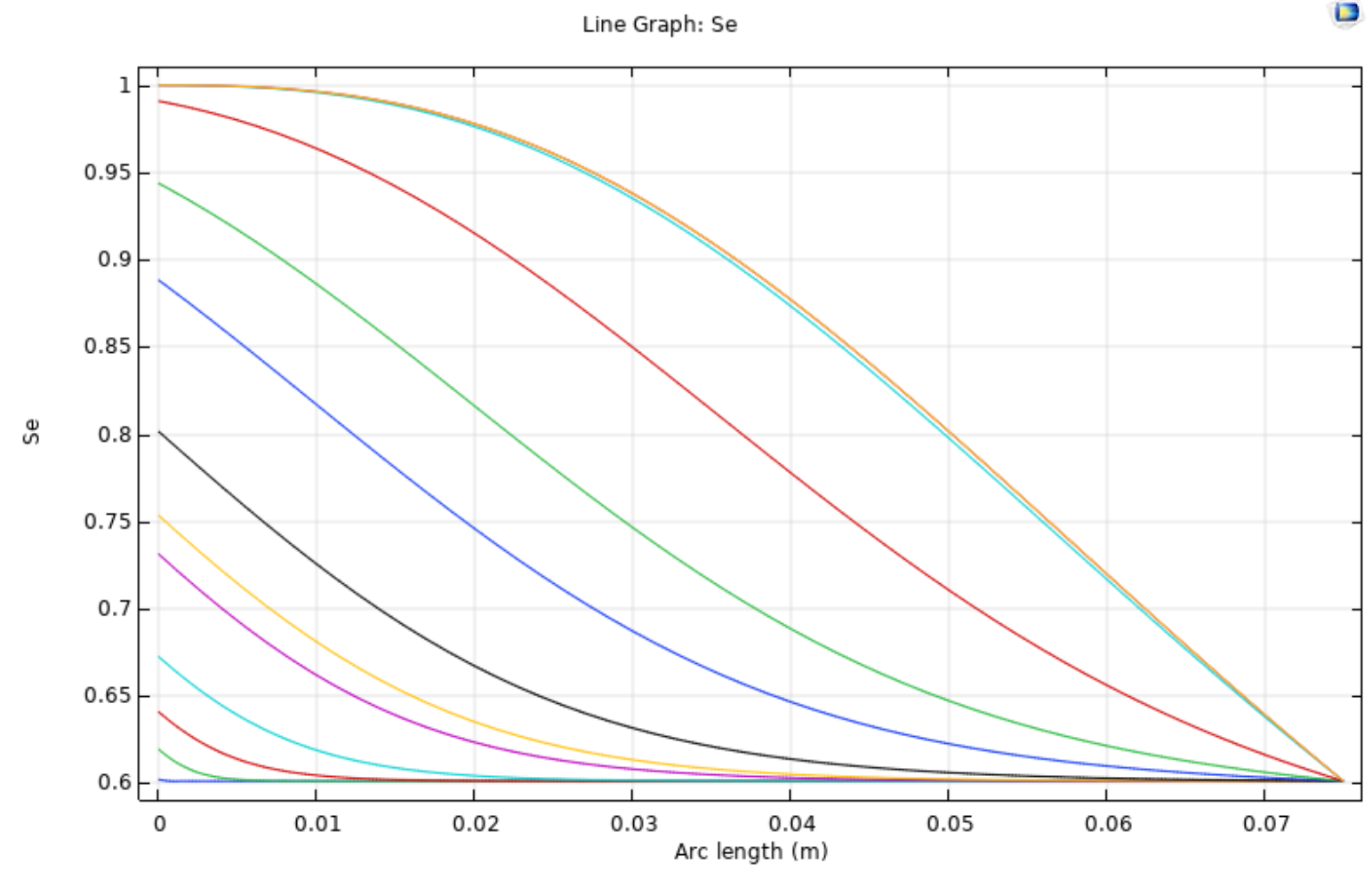

Figure 5.3: As an example, the illustration is a COMSOL plot of Se (the effective saturation) as a function of the height of the $50 \mathrm{~mL}$ tube ( 0 is the base and 0.075 is the top of the tube). An initial saturation $\left(\theta_{s}\right)$ of 0.18 , and residual $\left(\theta_{r}\right)$ saturation of 0 . Each curve represents a different time. As time approaches infinity, the medium becomes saturated at the base of the tube (because there is a no flux boundary condition there. 
Using theory and results from the percolation experiment, the mathematical formulation of solute transport in the sandy soil can be depicted as:

$$
\frac{\partial(\theta c)}{\partial t}=-\nabla \times J_{s}
$$

where the change of the solute concentration in time is equal to the negative gradient of the solute flux.

\subsubsection{Effects of Soil Moisture}

\section{Response of N-doped PPy Sensors in Sand}

2mm Sand Following calibration tests, the N-doped PPy sensors were tested for their amperometric response to LOW, MED, and HI NO3- concentrations in quartz sand containing various moisture levels. There was a strong positive correlation between soil moisture content and soil peak current sensor readings over a span of $0 \%-28 \%$ soil moisture in all spiked soils. Data in figures produced polynomial relationships between peak current responses in complete dry and field capacity. These data produced 4 degree polynomial fits with $\mathrm{R}^{2}$ values 0.90 .

The overall goal of the experiment was to understand which variables effect the SR values. When these predictors were not controlled, none of the variables were statistically significant. However, when we control for concentration, OM and $\theta_{m}$, the remaining variables were found to be significant and able to explain more than $90 \%$ of the variation in the dependant variable. The table 5.2 studies which variables are significant and by controlling the listed predictors, more than 90 percent of the variation can be accounted for.

A less complex 2 degree polynomial relationship was observed between $13 \%$ and $28 \%$ soil moisture content in $2 \mathrm{~mm}$ quartz sands. This data also reveals an increase in sensor 


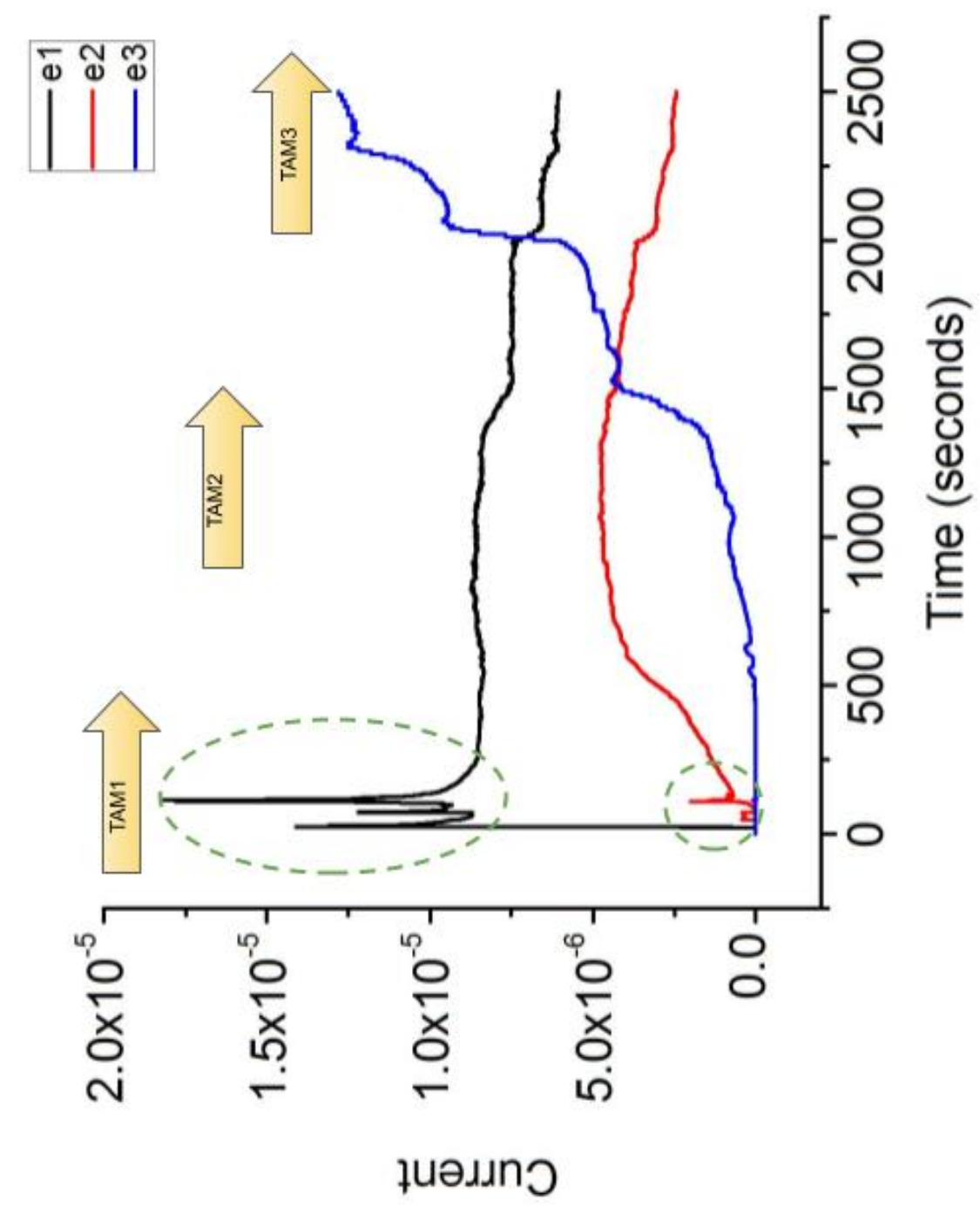

हี

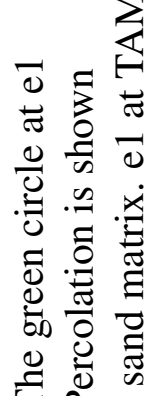

E

递它

흥 즐

㞯

氙 Ð

을

웜

휴윯

는 0

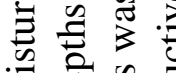

으ㅇㅝㅡㄹ

¿

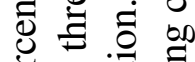

䒕至

$\infty$ ঠृ क 는

ㄴ. 을

요용

.응

㐘

ᄋ ठ

ฮ

렁

สำ

.

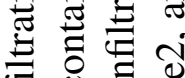

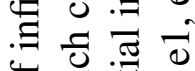

을 壬

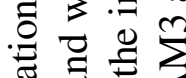

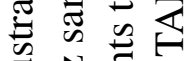

실 웅

三 సี

污

的范导

尝导芯 
Table 5.2: Trendline fitting in $2 \mathrm{~mm}$ Sandy Soil SR model

\begin{tabular}{lllll}
\hline Conc. & OM (\%) & $\begin{array}{l}\theta_{m} \text { range } \\
(\%)\end{array}$ & $\begin{array}{l}\text { Polynomial } \\
\text { (degree) }\end{array}$ & $\mathrm{R}^{2}$ \\
\hline HI & 0 & $0-28$ & 4 & .96 \\
HI & 2 & $0-28$ & 4 & .98 \\
MED & 0 & $0-28$ & 4 & .95 \\
MED & 2 & $0-28$ & 4 & .93 \\
LOW & 0 & $0-28$ & 4 & .96 \\
LOW & 2 & $0-28$ & 4 & .91 \\
HI & 0 & $13-28$ & 2 & .97 \\
HI & 2 & $13-28$ & 2 & .97 \\
MED & 0 & $13-28$ & 2 & .96 \\
MED & 2 & $13-28$ & 2 & .94 \\
LOW & 0 & $13-28$ & 2 & .95 \\
LOW & 2 & $13-28$ & 2 & .93 \\
\hline
\end{tabular}

accuracy as $\theta \mathrm{m}$ approaches field capacity and flooded conditions. This supports a theoretical model, given that more nitrate ions are released in the presence of moisture, as the availability of soil nutrients are highly dependent upon soil moisture [17]. As shown in the Table 5.2, the increased OM content decreases the $\mathrm{R}^{2}$ value of the trend-line, however, despite the added OM the fit model retains its $\mathrm{R}^{2}$ value above .90 .

$710 \mu \mathbf{m}$ Sand The amount of sensor drift from baseline readings of LOW, MED, and $\mathrm{HI}$ concentrations begin to decrease at field capacity and flooded conditions. Between $0 \%$ and $18 \%$ soil moisture content the peak currents followed a linear pattern in HI and MED concentrations. In $710 \mu \mathrm{m}$ particle size sand the accuracy of the sensors submerged in this particle size was greatly diminished. As moisture level reached field capacity, a slight improvement in accuracy of SR readings were observed in $710 \mu \mathrm{m}$ particle size sand. It was assumed the smaller particle size would contribute to smaller micro and macro pore space between particles and the volume of solute would completely saturate all pore spaces. 


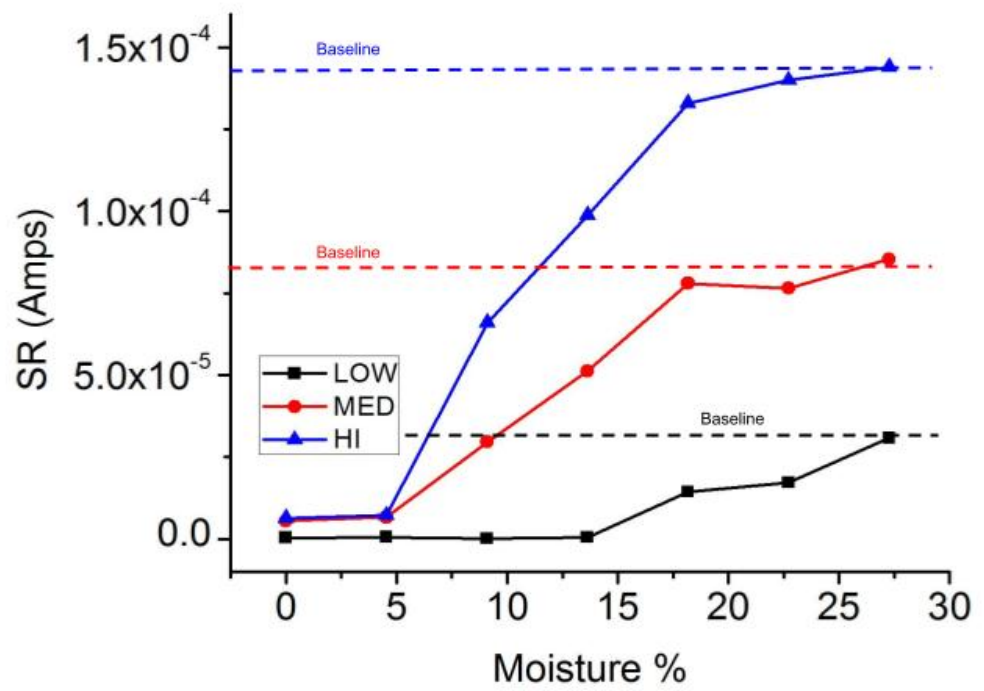

Figure 5.5: Illustration of $\theta_{m}$ effect on SR at three different nutrient concentrations. The moisture ranges from $0 \%$ (dry) to $28 \%$ (field capacity). Observe accuracy increases as moisture level rises.

\subsubsection{Effects of Soil Organic Matter}

As seen in figure 5.5, the general behavior shows the SR declines as OM content increases. The $710 \mu \mathrm{m}$ graphs followed this behavior However, higher moisture content seemed to improve accuracy at $\mathrm{HI}$ concentrations. At 2 and 5 percent $\mathrm{OM}$, the $\mathrm{OM}$ is highly dispersed through the sand column. Therefore its ability to absorb moisture influences the random rate and direction of solute percolating through the sand matrix. This behavior is highlighted in $2 \mathrm{~mm}$ graphs of HI, MED and LOW concentrations. At 10 percent $\mathrm{OM}$, the sand matrix has become dominated by $\mathrm{OM}$ and retains solute as it percolates through the sand matrix. Therefore, in $2 \mathrm{~mm}$ sand where pore spaces are quite large, OM content induced minimal influence on the SR at high percent moisture. In 2 mm sand containing 0-10 percent OM, the amount of sensor drift observed was less than $.25 \mathrm{E}^{-4}$ in $\mathrm{HI}, 3 \mathrm{E}^{-5}$ in MED and $3 \mathrm{E}^{-5}$ in LOW concentrations. 

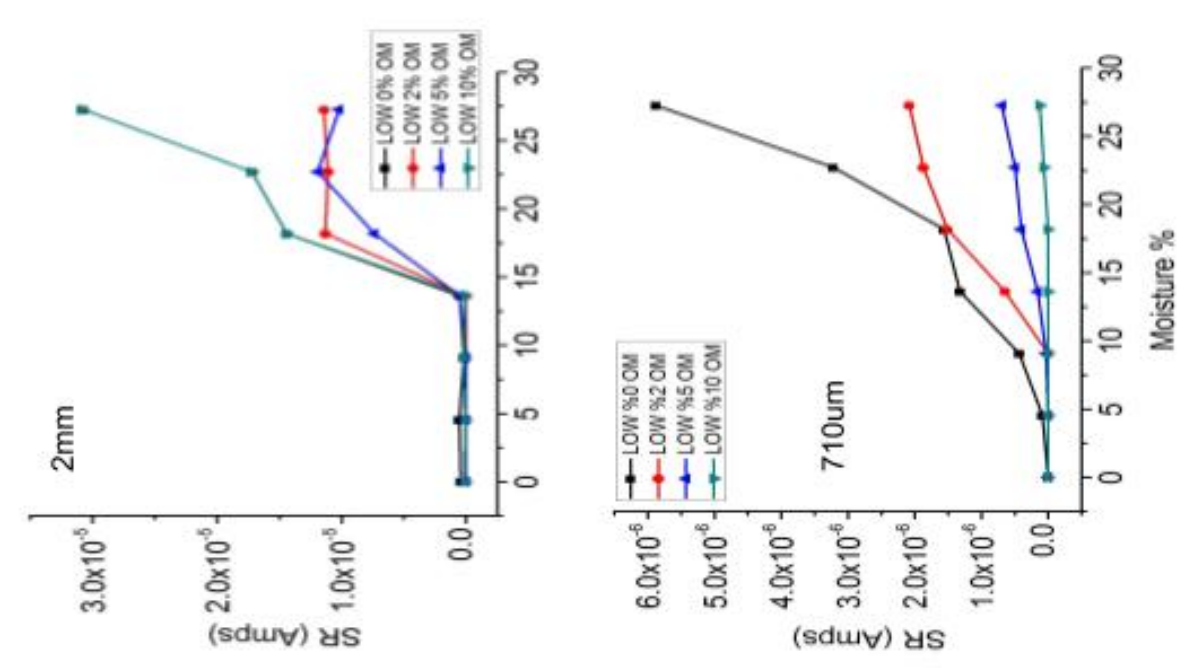

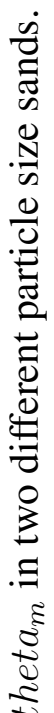
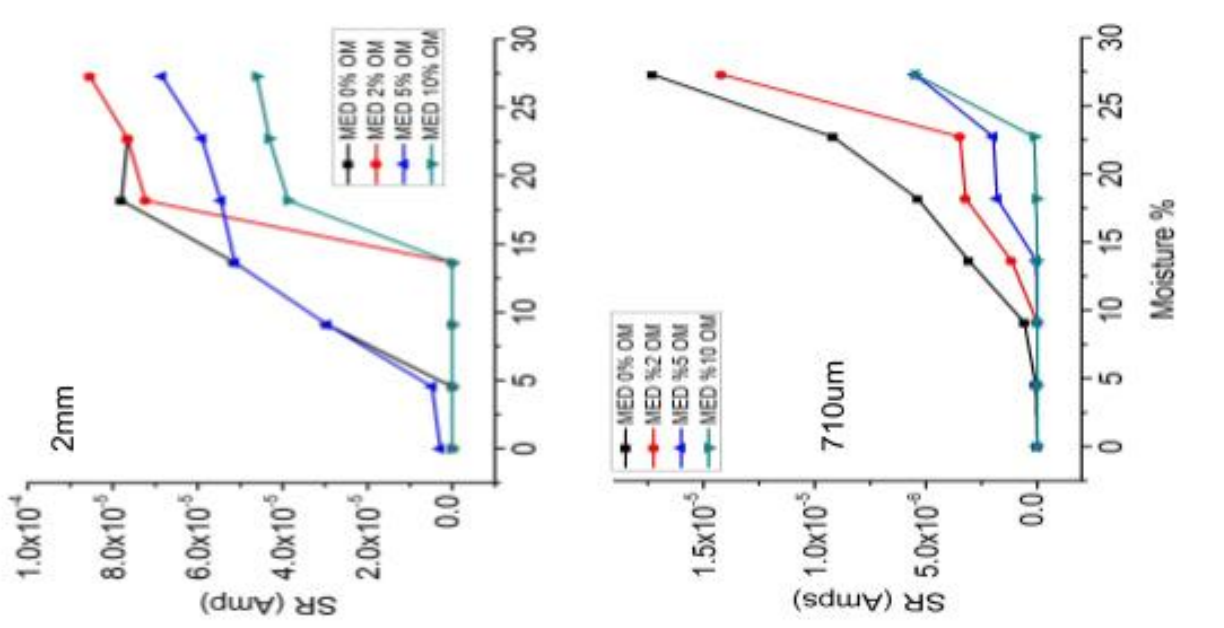

0
0
0
0
0
0
0
4
0
0
0
0
$\vdots$
$\Xi$
0
0
0
0
0
0
0
0
0
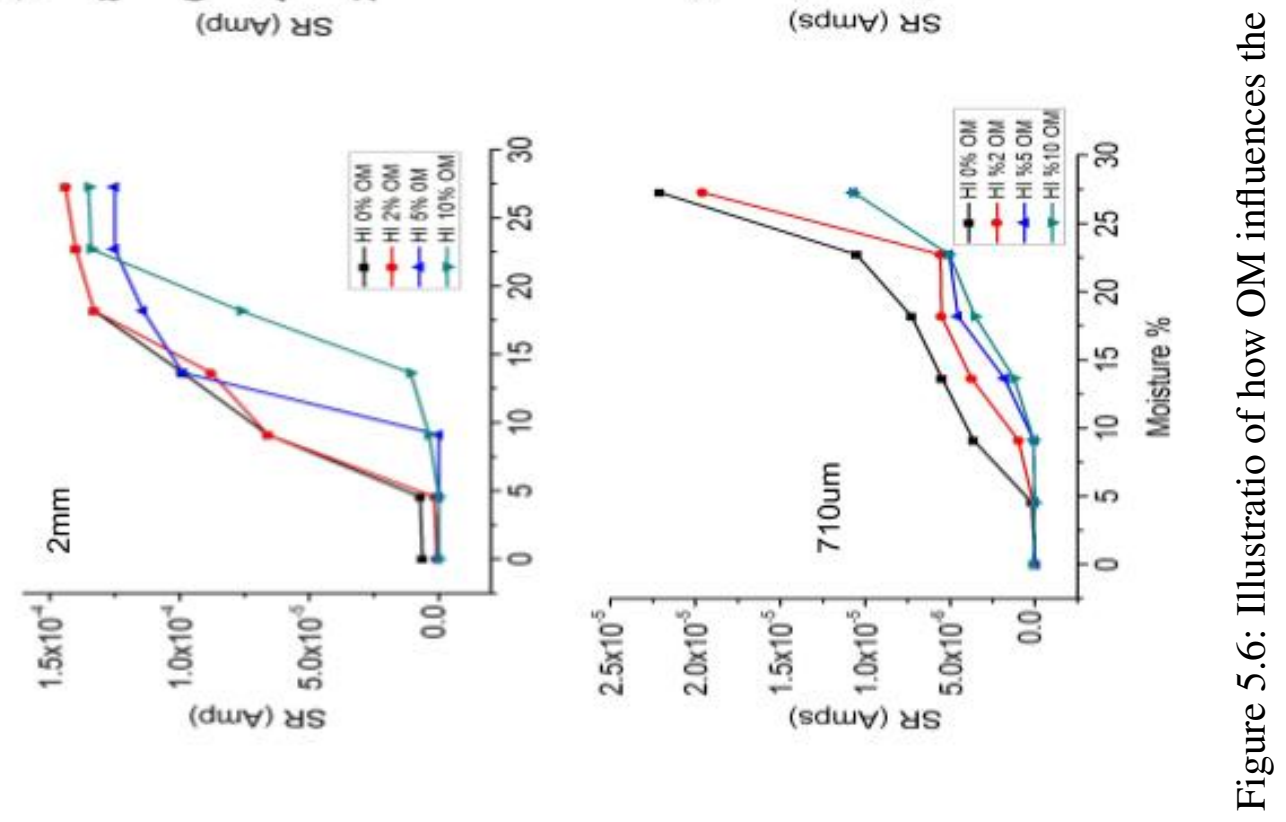


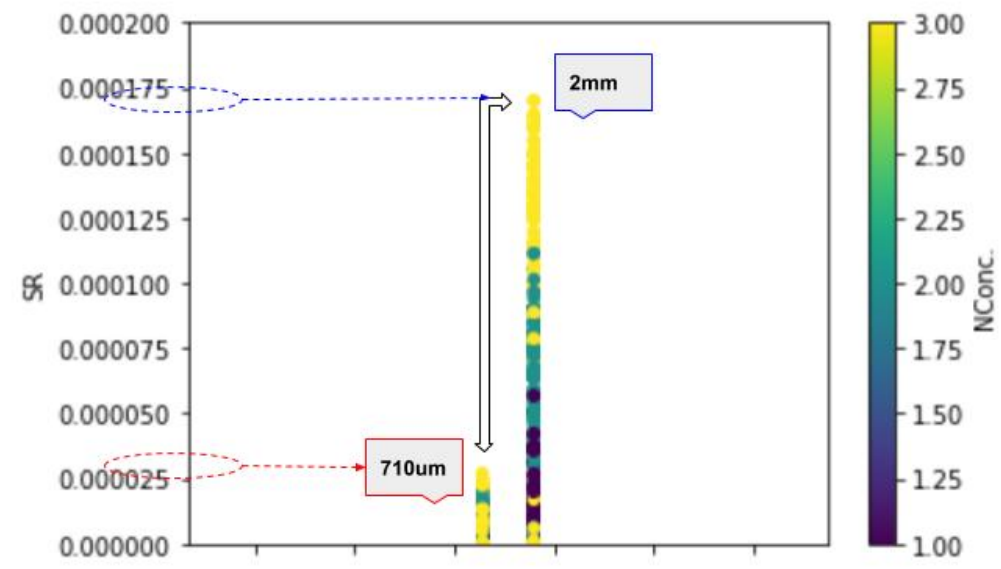

Figure 5.7: Illustration reveals the variation in SR values as a result of particle size. More than $70 \%$ drift in SR was observed in smaller particle sized sand.

\subsubsection{Effects of Soil Particle Size}

2mm Particle Size Quartz Sand In $2 \mathrm{~mm}$ particle size quartz sand, larger peak current difference between LOW and $\mathrm{HI}$ sand groups compared to that of $1 \mathrm{~mm}$ particle size were observed, especially as soil moisture approached field capacity. At field capacity sensors buried in $710 \mu \mathrm{m}$ particle size sand produced peak currents nearly $70 \%$ less than those submerged in $2 \mathrm{~mm}$ particle size sand (Figure 5.6). It was postulated that the peak differences can be due to the fact that larger particle size sand has larger pore space between particles, therefore a larger volume for air-water accumulation and solute transport within the soil matrix.

$710 \mu \mathrm{m}$ Particle Size Quartz Sand In $710 \mu \mathrm{m}$ particle size quartz sand, a large difference between baseline and sensor raw readings were observed in comparison to those readings from $2 \mathrm{~mm}$ sand. Since the smaller particle size would contribute to smaller 
micro and macro pore space between particles, it was assumed that a lesser volume of solute would completely saturate all pore spaces, thereby providing more accurate SR responses when $\theta \mathrm{m}$ values were smaller. However, the data obtained from the sensors submerged in $710 \mu \mathrm{m}$ sand disproved this assumption. Further postulation points towards scaling the electrode system in a manner that would accommodate the micro-pore space of finer textured soils and smaller particle sizes.

\subsubsection{Effect of Sensor Placement and TAM Measurements}

Sensor placement and TAM experiments were conducted to understand their influence on SR. The experimental design was similar to that of the infiltration and percolation experiment discussed in a prior section. This included three different electrode depths, e1, e2, and e3, and three unique times after applied moisture contents (TAM1, TAM2, and TAM3). Instead of applying various mass water contents of conductive solutions, three different nitrate concentrations were applied, HI, MED, and LOW. As shown in graph 5.7, e1 at TAM1 produced the most SR values, however, e2 and e3 produced no signal during TAM1. During TAM2, SR in e2 and e3 was first observed. It was observed that higher moisture content levels were needed to percolate towards e3. SR at e3 was only observed in TAM 's 2 and 3 when 18 percent moisture was applied. As the moisture increased from 18 percent, so did the accuracy of the SR in e3. Over all, the accuracy improved over time and increased moisture in e 2 and e 3 between 18 percent moisture and field capacity.

\subsection{Lead Length Measurements}

The purpose of large lead measurements was to understand the effects of lead length on sensor output. When scaling a sensor platform design for practical and precise large 


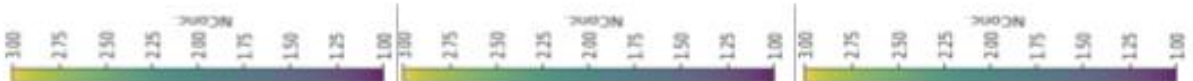

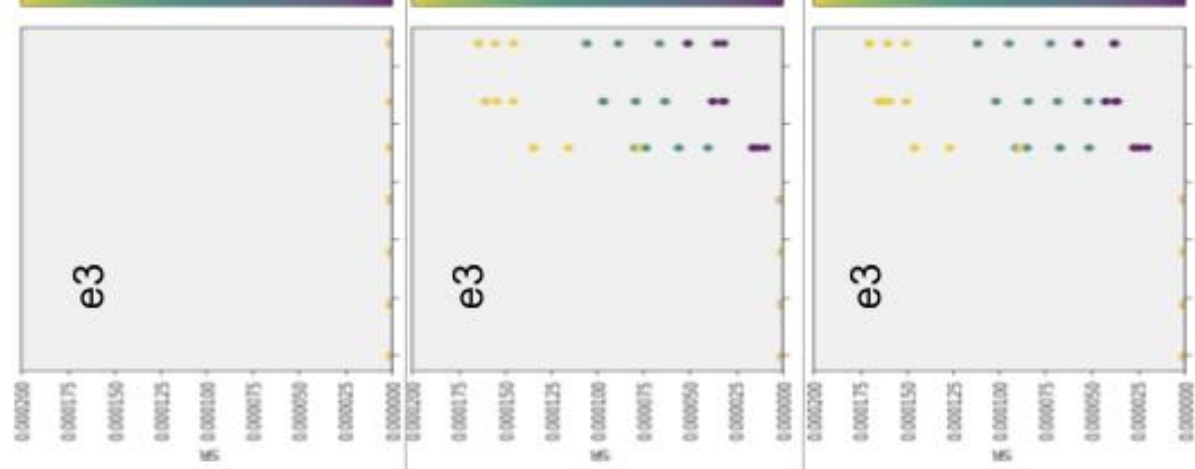

空

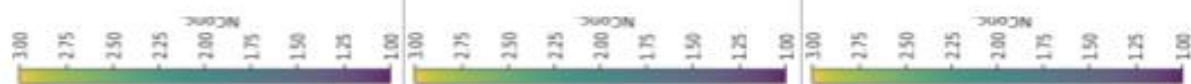

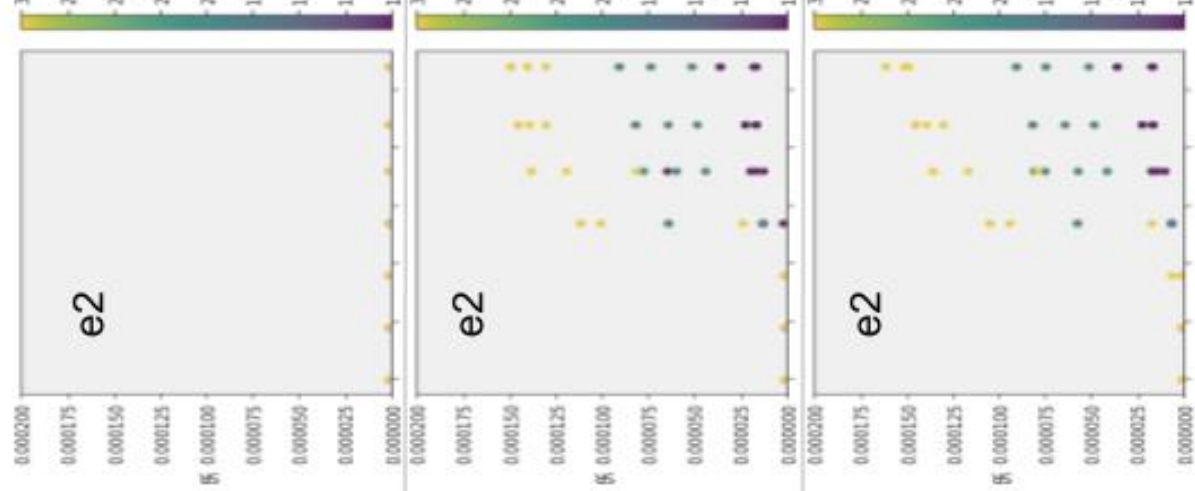

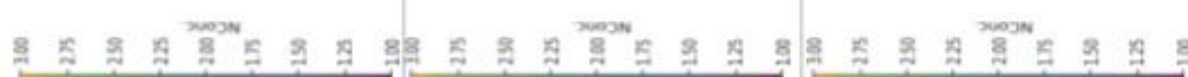
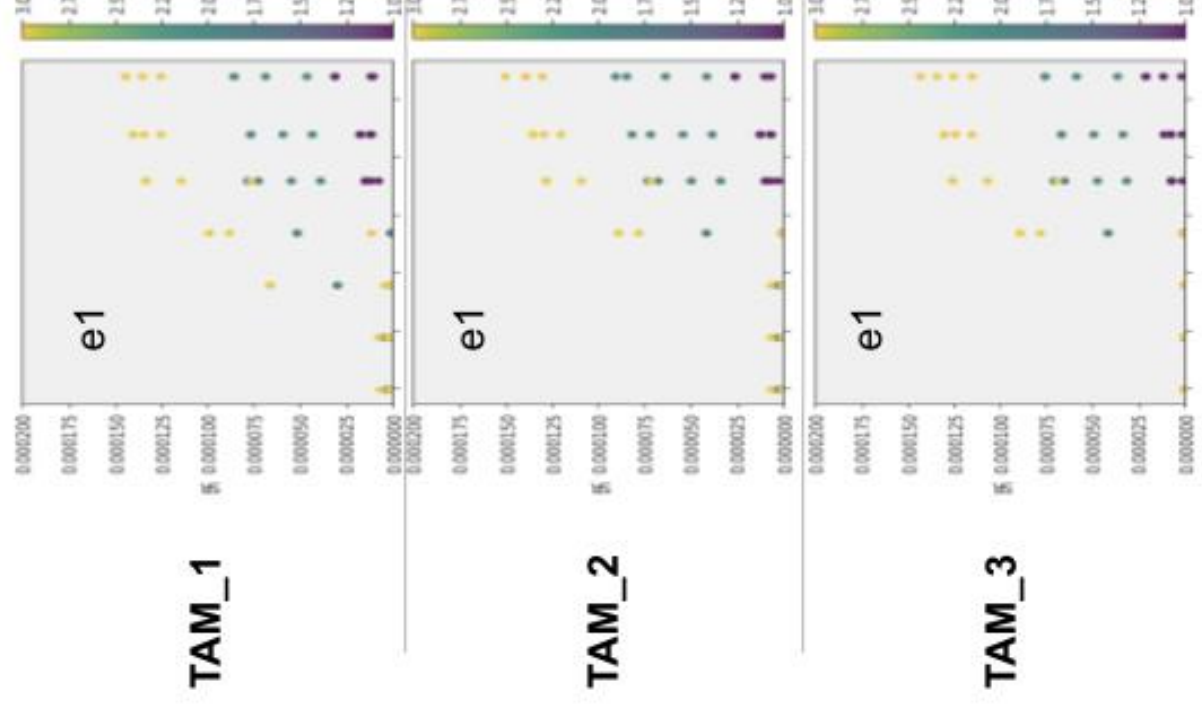

$\sum_{1}^{m}$

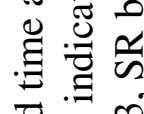

言

๙ิ)

ஜ

ขิ

i

$\underbrace{\infty}_{\infty} m$

苍完

ठ छ

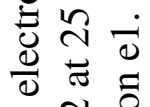

๑ ข

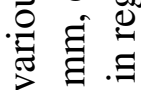

⼆ㅡㅇ

ฮै

స్ ญ

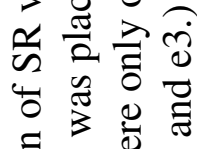

힐

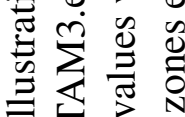

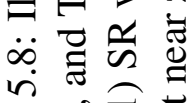

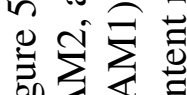

它这芎苛 


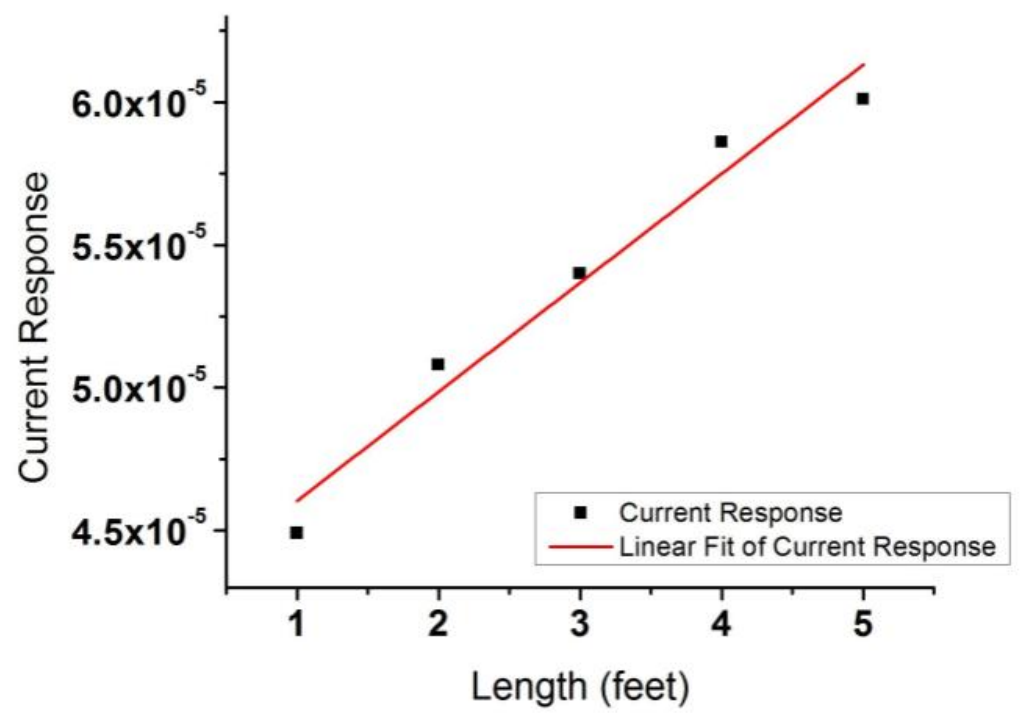

Figure 5.9: Illustration of peak current vs the lead length in feet.

scale soil nutrient sensing, the following challenges may arise: 1) unbalanced sensing performance at different locations, and 2) noisy sensing incurred by signal cross-talk. Sensors further away from power source experience signal loss and large current variation due to unbalanced signal routing. Figure 5.8 illustrates there is a change in peak current vs the change in length (feet) and interference from neighboring sensors can largely deviate the sensing of a target sensor. Over a span of five feet, the current peaks to followed a linear slope of $3.82 \mathrm{E}^{06}$ and had an $\mathrm{R}^{2}$ value of .97 . The equation of the form is:

$$
I(A)=3.82 E^{-06} L L+4.6 E^{-05}
$$




\subsection{Modeling the effects of SR}

Overall, through the above experiments, a quantitative response between $\mathcal{Y}$ and the predictors $\mathcal{X}$ was observed for the purpose of prediction and inference. This function can be written as:

$$
\mathcal{Y}=f(\mathcal{X})
$$

the data derived from the experiments allowed a generalized model to be deduced which explained the predictors $\mathcal{X}$ effect on SR which. This can be depicted as:

$$
S R=f\left(\theta_{m}+O M+E_{\text {place }}+T A M+P_{\text {size }}\right)
$$

Prediction $\mathrm{ML}$ was utilized for predicting the response of SR in the form $\hat{\mathcal{Y}}=\hat{f}(\mathcal{X})$. Here $\hat{f}$ represents the estimate for $\mathrm{f}$, and $\hat{Y}$ is the resulting prediction for $\mathrm{Y}$. In the case of the above parameters, this can be written as:

$$
\begin{aligned}
& E(S R-\hat{S R})^{2}=\left[f\left(\theta_{m}\right)-\hat{f}\left(\theta_{m}\right)+f(O M)-\hat{f}(O M)+f\left(P_{\text {size }}\right)-\hat{f}\left(P_{\text {size }}\right)\right. \\
& \left.+f\left(E_{\text {place }}\right)-\hat{f}\left(E_{\text {place }}\right)+f(T A M)-\hat{f}(T A M)+\text { Var } \epsilon\right]
\end{aligned}
$$

where $E(S R-\hat{S R})^{2}$ represents the expected value of the squared difference between the predicted and actual SR value, and Var $\epsilon$ represents the variance of the error term. The experiments revealed much of the data to be non linear. Given the quantitative nature of the target variable, the regression approach was taken when selecting algorithms. For this work, OLS, polynomial regression, Decision Tree and Random Forests were the ML algorithms selected and evaluated against one another. The performance of the algorithms were evaluated based on the values of $\mathrm{R}^{2}$ and MSE score.

Based on the table 5.3, the results showed the polynomial regression (PR) model performed well with MSE value of 32.761 and a $\mathrm{R}^{2}$ value of 84.7 . This was followed by 
Table 5.3: Algorithm Evaluation in SR model

\begin{tabular}{lll}
\hline Algorithm & $\mathrm{R}^{2}(\%)$ & MSE \\
\hline OLS & 40.5 & 84.875 \\
DT & 60.4 & 72.938 \\
Random Forests & 77.1 & 49.921 \\
Poly. Reg. & 84.7 & 32.761 \\
\hline
\end{tabular}

the Random Forests (RF) model which produced an MSE of 49.921 and a $\mathrm{R}^{2}$ value of 77.1. The prediction of models OLS and DT were not as precise as those of RF and PR and therefore required further improvement. Overall, the RF and PR algorithms can be successfully used to predict SR values.

Inference In the case of inference, statistical learning was implemented to understand the relationship between $\mathcal{Y}$ and $\mathcal{X}$. More specifically, how the $\mathrm{SR}, \mathcal{Y}$, changes as a function of soil heterogeneity, $\mathcal{X}$. The predictors found to be substantially associated with SR were $\theta_{m}$ and $P_{\text {size }}$. It was established that by increasing the predictor value $\theta_{m}$, the accuracy of the SR could also be increased. The same with $\mathrm{P}_{\text {size }}$, decreasing this further increased the drift and decreased accuracy of SR. OM had a similar effect on SR, by increasing OM the drift of the sensor increased. However, the behaviors of hydraulic dispersion outweighed the effects of OM as $\theta_{m}$ increased to field capacity and flooded conditions.

\subsection{Summary}

In summary, highly sensitive and selective nitrate sensors were produced by using electrochemical $\mathrm{NO}_{3}^{-}$doping of polypyrrole. The sensors were calibrated and showed linear response to nitrate between the ranges of $1 \mu \mathrm{M}$ to $60 \mu \mathrm{M}$ with a sensitivity of -1.856 $\mathrm{E}^{-06}$. When buried, the sensors demonstrated response to nitrate concentrations spiked 
in quartz sand. Sensor readings were most significantly $(\mathrm{p}<0.05)$ affected by the $\theta \mathrm{m}$, then followed by OM, $\mathrm{P}_{\text {size }}, \mathrm{E}_{\text {place }}$, and TAM respectively. Infiltration and percolation measurements allowed visualization of solute transport throughout the sandy soil matrix. The percolation measurements identified e 1 at TAM1 and e 2 and e 3 at TAM 3 to be the optimal times to obtain a soil measurement, considering the placement of the electrodes and time after 18\% moisture was applied to the sandy soil. Initially a linear model was fit, but it did not explain the variance in the sensor response adequately. The $\mathrm{R}^{2}$ value was only 0.40 as shown in Table 5.3. During the exploratory data analysis, we noticed that, for fixed/constant values of $\mathrm{N}$ concentration and $\mathrm{OM}$, the rest of the variables can be used to explain the variation in the sensor response satisfactorily. In fact, the $\mathrm{R}^{2}$ values with polynomial regression were above 0.9 for each of the subsets of the data. Hence, non-linear models such as decision tree, random forests in addition to polynomial regression were implemented. When the full dataset was employed, polynomial regression derived the best model with an $\mathrm{R}^{2}$ value of 0.84 (Table 5.2). Overall, sensor accuracy improved in all spiked soils as soil moisture approached field capacity and flooded conditions. A linear fit was produced which explained the relationship between $\mathrm{SR}$ as lead length increased and the slope of the form was derived as $3.82 \mathrm{E}^{-06}$ with an $\mathrm{R}^{2}$ value of .97. Overall, the soil heterogeneity study on SR help to produce optimized models for predicting SR and accounting for majority of the variance due to predictors. 


\section{CHAPTER 6}

\section{FABRICATION OF SENSOR SHEETS AND PODS}

\subsection{Overview}

Recent advances in electrical engineering has influenced precision agriculture by providing sensor technology. In addition to such technologies, Nitrogen $(\mathrm{N})$, from fertilizer application is the leading method for improving yield production in agriculture. $\mathrm{N}$ fertilizer also remains one of the largest input to surface and groundwater contamination, resulting in environmental and human health degradation. This chapter presents the fabrication of soil sensor sheets and pod systems and discusses the integration of electronics with these systems. It also discusses the fabrication and characterization of nitrate $\left(\mathrm{NO}_{3}^{-}\right)$doped polypyrrole (PPy) ion selective electrodes (ISE's). It highlights the role of electropolymerization (EP) time and recipe concentration on the polymer membrane film growth and performance. Scanning electron microscopy (SEM) technology was used to observe surface morphology and characterize the sensor device.

\subsection{Introduction}

Majority of nitrogen $(\mathrm{N})$ found in soils is contributed by animal waste and fertilizers. The most common form of nitrogen that is plant-available in soils is nitrate $\left(\mathrm{NO}_{3}^{-}\right.$. Element nitrogen can also be present in soils as ammonium $\left(\mathrm{NH}_{4}^{+}\right)$, nitrite $\left(\mathrm{NO}_{2}\right)$, and or organic matter. As nitrate is a major limiting macro-nutrient for plant growth, it is often highly explored in detailed by agriculturalist. The high mobilizing property of the nitrate ions allows it to easily maneuver through soils, therefore leaching and running off into waterways. $\mathrm{N}$ in excess is subject to runoff and leaching into surface and groundwater during rainfall and irrigation. High nitrogen concentration in drinking water sources im- 
poses a threat to humans when nitrates runoff into surface water; the buildup can lead to a polluted state toxic to marine life known as eutrophication. In [105] and, [106] researchers in China addresses special issues of eutrophication such as the increased activity of algal blooms and fish kills as well as investigate nitrate leaching under intensive vegetable garden production patterns. The conclusions indicate the need for innovative technology that can better monitor soil quality for the protection of the environment. The standard laboratory techniques for determining total $\mathrm{N}$, ammonium, nitrate, and nitrite are still the most commonly used. In, [107] these techniques are listed along with recommended test procedures for soil chemical analysis. These include potentiometric methods - explored in, [23] [108] [109] [110] cadmium reduction, [111] [112] [113] ion chromatography, [114] [115] [116] steam distillation [117] [118] and the hot KCL removal of $\mathrm{N}$ [119] [120] [121]. Techniques for in-situ $\mathrm{N}$ determination using Time Resolved Raman Spectroscopy (TRRS) has been explored in [122], however this technique is not the most popular among farmers. Although these techniques are highly accurate, they consists of expensive and time consuming lab procedures that can in some instances take days to obtain results. Nevertheless, commercially available technology for determining soil nutrients are increasingly becoming popular for its ability to allow quick and easy measurement of soil constituents that aid plant growth and those that cause aquatic pollution. However, these sensors are point of use only, meaning they can only account for concentration present in the area of the sample. Also, the technology is expensive for even most commercial farmers, therefore farmers can only afford one sensor unit per acre. One sensor per acre can prove inefficient because farmland can contain various terrain which can cause nutrient concentration to also vary among different areas. For these reasons, farmers are seeking alternative and more convenient methods to monitor the environment. Specifically, an affordable sensing platform is needed that will allow accurate real-time quantification of spatially varied soil nutrient concentration 

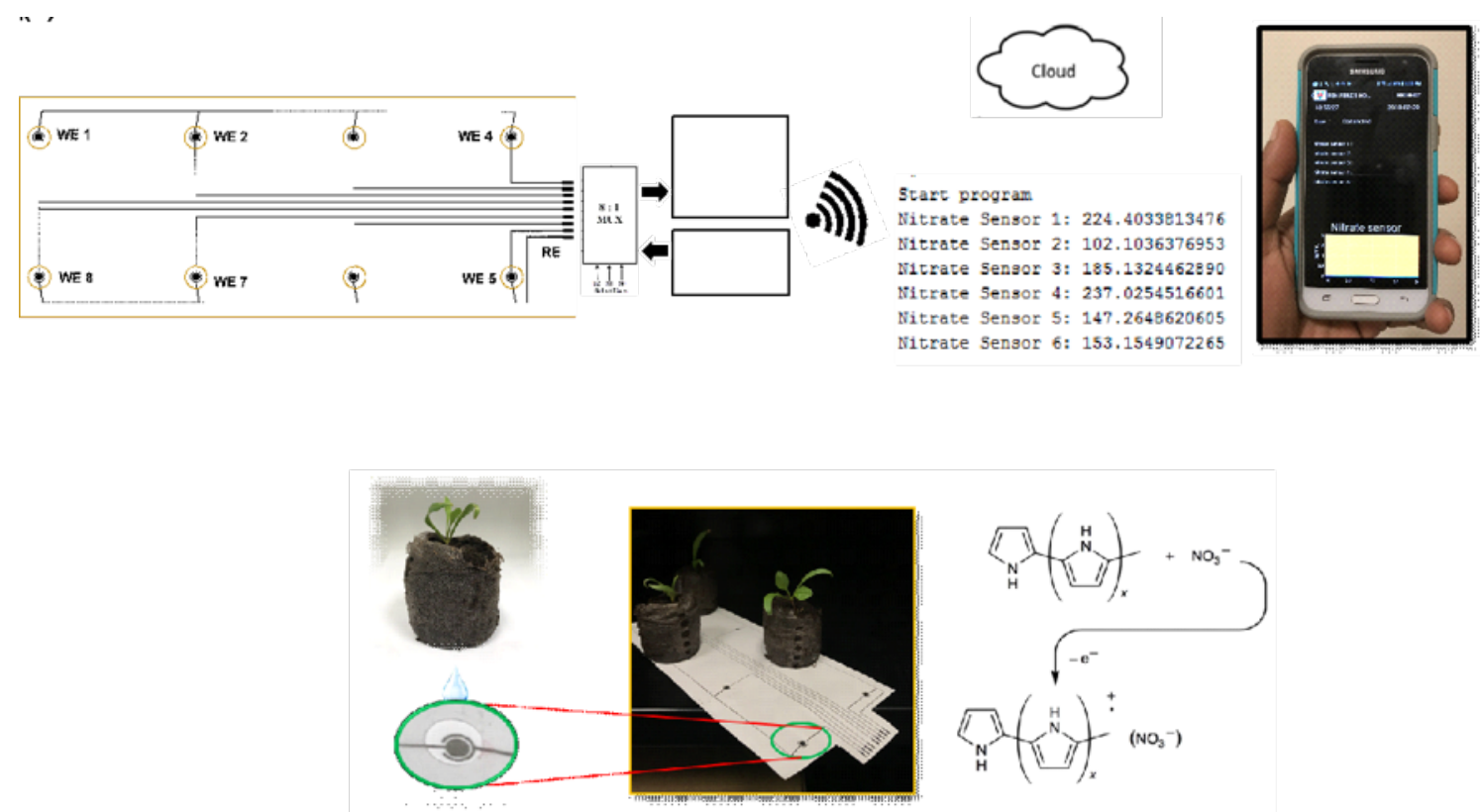

(b)

Figure 6.1: a) Layout of the IoT enabled soil sensor system. b) Seedling pellets on nitrate sensor sheet

for precision agriculture. As defined by the United States Department of Agriculture (USDA), precision agriculture is an information based management system that is site specific and uses one or more of the following sources of data: soils, crops, nutrients, pests, moisture, or yield, for optimum profitability, sustainability, and protection of the environment [123]. The position and data dependent systems divide farmland into zones and gathers farm data inside the zones such as soil moisture, $\mathrm{pH}$, and soil temperature. The data is sent to cloud via secure algorithm that monitors $60 \mathrm{ft} \times 60 \mathrm{ft}$ zones to suggest best farming practices based on past and future predictions of soil quality and crop production. Unfortunately for large scale farms, high density subsurface sensing of soil chemistry has not been fully employed.

Recent advances in IoT have opened up the possibilities for networking arrays of sensors in order to obtain stochastic data on various soil components. The internet of things (IoT) is a global network of interrelated devices and objects which uses unique identi- 
fiers, such as an IP address, and the ability to transmit data over a network. Total number of IoT connections is expected to grow from 5 billion to 27 billion by year 2024. IoT has been deployed in agricultural settings for precision agriculture [124] [125] [126] [127] [128] [129] [130] [131] [132] [133] [134]. These IoT systems offer a range of services to farmers such as soil water management, environmental weather parameters, and tree and crop monitoring. IoT has given the farmer the ability to monitor and manage agricultural production via cellular device. To date, wireless sensor networks (WSN's) have been deployed in environmental and agricultural fields for numerous applications such as to manage water resources [135], manage product storage facilities, determine optimal harvest time, characterize crop growth, and predict fertilizer requirements. Wireless sensor network has been deployed in [135] to monitor water content, temperature, and salinity of soil at a cabbage farm located in a semi-arid region of Spain. Temperatures at various positions in a feed warehouse were monitored using a wireless sensor network in [136]. WSN using Zigbee has been used to monitor a greenhouse environment [137] [138] [139] [140]. The energy limitations of wireless sensor networks also have been a focus [141].

\subsection{Materials and Methods}

Sensor sheets, prepared in-house, were printed onto a photopaper substrate with aqueous ink, which was formed using a combination of solvents [142]. The ink is composed of well-dispersed silver nanoparticles $(10 \mathrm{~nm})$ with a silver concentration of $20 \mathrm{wt} \%$, viscosity of $9.5 \mathrm{cP}$ and a surface tension of $36 \mathrm{mN} \mathrm{m}^{1}$ which met inkjet printer (MFCJ680DW) requirements. The nanoparticles were protected by a capping layer of poly (N-vinylpyrrolidone) (PVP). Pyrrole(98\%), sodium nitrate $\left(\mathrm{NaNO}_{3}^{-}\right)$, and $\mathrm{PBS}$ buffer solution were purchased from Sigma and were all of analytical grade. Pyrrole, being 
light sensitive, was refrigerated in the dark. Sensors were calibrated using $0.1 \mathrm{M} \mathrm{NaNO}_{3}^{-}$ stock solution in PBS (pH 7.2). Separate solutions of $\mathrm{NaNO}_{3}^{-}(0.4 \mathrm{M}$ and $0.1 \mathrm{M})$ were prepared for electropolymerization and electrode conditioning. Sensor response to target ions was evaluated using a conventional one compartment three electrode electrochemical cell. $\mathrm{A} \mathrm{Ag} / \mathrm{AgCl}$ double junction electrode was used as a reference electrode, a platinum counter electrode and the screen printed working electrode.

Clean-room Fabrication In addition to the fabrication of printed sensor sheets, gold electrodes were also fabricated in clean room environment to serve as a gold standard for measurements. Six inch silicon oxide wafers were first cleaned with acetone, IPA, and DI water then dried with a nitrogen gun. The clean wafer was then placed on spin coating apparatus, where positive photo resist (PR) was applied. Immediately after spin coating the wafer experienced a bake of $90 \mathrm{C}$ for five minutes then set to cool in ambient temperature. After cooling, the wafer containing PR was loaded into mask aligner for photlithograpghy. The wafer received an exposure of approximately $17.8 \mathrm{~J} / \mathrm{s}^{-1}$ for 5.8 seconds. After, the wafer was submerged in developer solution for approximately 2 minutes. After developing, the wafer was washed then observed under a stereomicrscope. Following, the wafer experienced evaporation deposition of $20 \mathrm{~nm}$ chrome and $100 \mathrm{~nm}$ of gold respectively. After deposition the wafer was subject to lift-off then cleaned with acetone, IPA, and DI water. Lastly, I-V characteristics were observed in a ferrous solution to gauge the viability of the fabricated gold electrodes.

\subsubsection{Electropolymerization}

Polymerization of pyrrole doped with nitrate on screen printed and gold clean-room electrodes was performed electrochemically using a Princeton Applied Research PotentiostatGalvanostat (Model 263A). A constant current of $100 \mu \mathrm{A}$ were applied for duration of 
5-20 minutes on the working electrodes. After each cycle, $100 \mathrm{~L}$ were added to cell solution to measure sensor performance against various nitrate concentrations. Electropolymerization was conducted with three separate pyrrole concentrations $(1 \mathrm{M}, 0.5$ M, 0.1 M) mixed with $1 \mathrm{M}, 0.4 \mathrm{M}$ and $0.1 \mathrm{M}$ of $\mathrm{NaNO}_{3}^{-}$. All solutions were prepared immediately prior to polymerization. Pyrrole is sensitive to ambient light and atmospheric oxygen.40 Therefore PPy solution was refrigerated and not exposed to light.

Soil Samples Three soil samples (L1, L2, and L3) were collected from three $12 \mathrm{~m} 2$ garden beds at Florida International University's Modesto Maidique Campus in Miami, Florida. Soils were identified via the USDA - NRCS's Web Soil Survey as limestonederived udorthents. They have a gravelly-loam texture, shallow to bedrock and poorly drained. Experimental plants were grown from seeds, twelve inches apart directly into garden bed soil, while controlled group were grown in 10 L pots of bare loamy soil with no added fertilizers or microbes. All groups were grown under 100\% sun with irrigation, weed and pest control done as needed. The Shurflow water pump transferred fluids from the storage tank to the sprayer with an inlet pressure of 2.06 bar (30 psi) and an output flow rate of 4 gallons per minute, providing test beds with water through DIG drip irrigation.

\subsubsection{Potentiostat Circuit}

The input range for the potentiostat circuit (Fig. 6.2) depend on the values of R1, C1, R2, R3 and the source of input (BATT or USB), there by having a direct control over the input voltage of the potentiostat. For instance, a 5 volt input will yield a linear input range in the 1 to $+1 \mathrm{~V}$ range. The circuit supports a power supply range of $3 \mathrm{~V}$ to $32 \mathrm{~V}$. Range of output current sensing is depended on the negative voltage to resistor R5. An applied voltage of $3.7 \mathrm{~V}$ yields a range of 150 to $120 \mu \mathrm{A}$ (at $5 \mathrm{~V}$ range is 210 to $65 \mu \mathrm{A}$ ). 


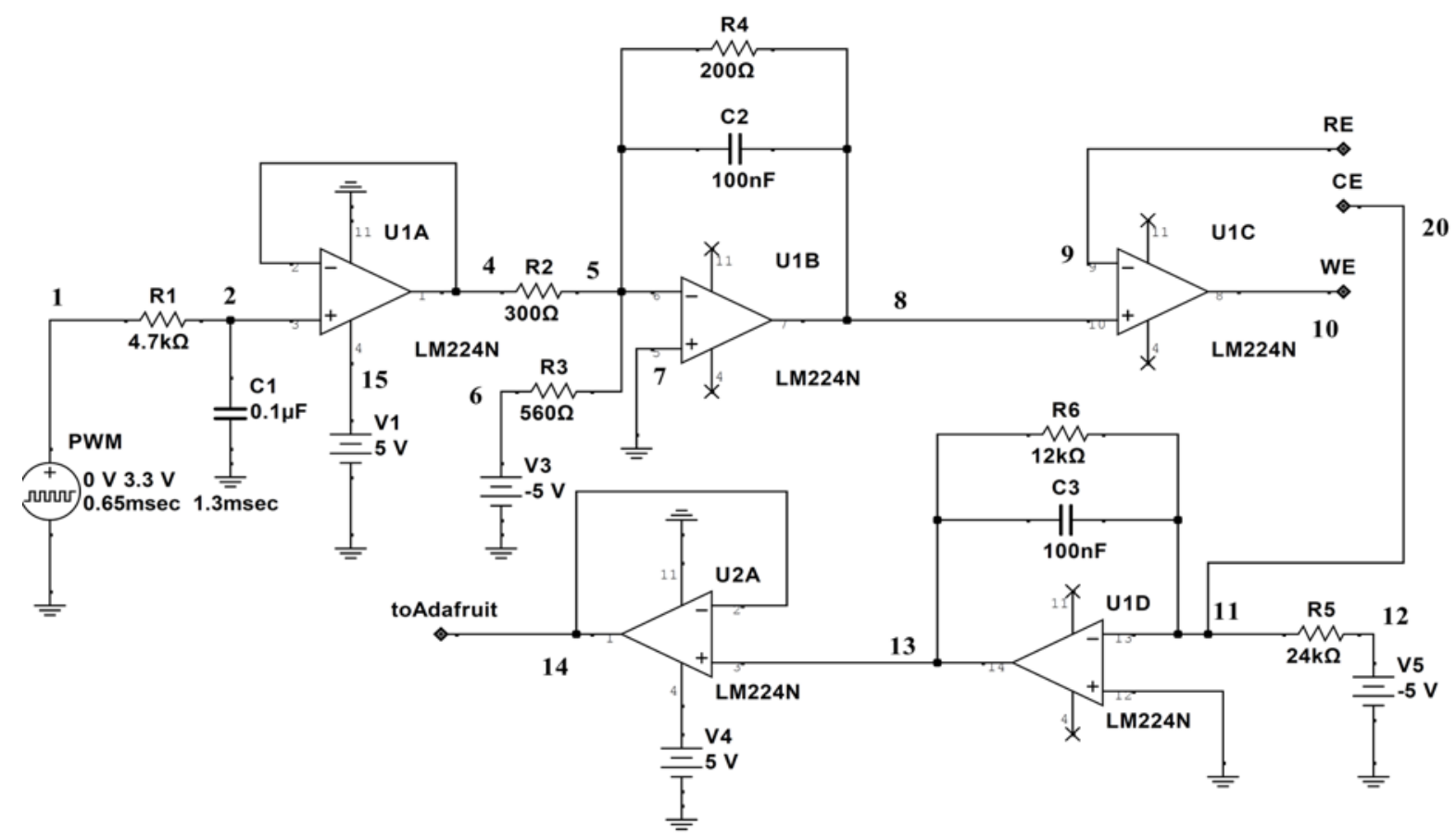

Figure 6.2: Linear sweep potentiostat circuitry

Alternatively, current sensing range can also be tailored by introducing variable resistors at positions R5 and R6. Power dissipation of the circuit was calculated as the products of voltage and current of all the power sources. Total power dissipation at ideal state is $50 \mathrm{~mW}$. The current drawn internally by op amp (X3) is $18.9 \mathrm{nA}$ and the current drawn internally by op amp (X5) were found to be $23.34 \mathrm{nA}$ ).

\subsection{Sensor Pods}

\subsubsection{Overview}

The rhizosphere microenvironment greatly influences the germination and production of plants. However, there is currently a lack of low-cost laboratory free techniques for fabricating such sensor integrated artificial structures to study biochemical behaviors of 
these micro-environments and their plants in real-time. Here, a sensor germination pod system, with micro-printed films, as a kind of plant-root microanalysis system has been sucessfully developed. This system integrates nutrient sensors and insulator structures that facilitate the germination, growth and phenotype analysis of plants. Here, the design and fabrication details of the plant-root microelectromechanical system (MEMS) and components which encompasses a microreactor root chamber integrated with in-house printed sensors are discussed. Further, this system was coupled with a microcontroller and the acquired data was remotely transferred and processed to provide a real-time nutrient uptake profile. The functionality of the device was validated through a study of legume plant germination, and observation of nutrient uptake. The fabricated sensor effectively measures nitrate and phosphate and the sensitivity of the nitrate and phosphate sensor was determined as $1.74 \mathrm{E}-5$ and $4.42 \mathrm{E}-7$ with R2 values of .99 and .95 both respectively. Plant-root micro-analysis and monitoring based on this principle has the potential of overcoming the cost, complexity, and real-time rhizosphere monitoring issues associated with traditional plant phenotype methods. This method and device have the potential to enable plant scientists to progress from conventional agar dish and pot plant phenotype techniques for real-time applications such as of root-pathogen interaction, drought-resistant plant variety screening, nutrient uptake efficiencies, and rhizosphere micro-environment monitoring.

\subsubsection{Introduction}

The complex and heterogeneous nature of the rhizosphere community provides a dynamic biochemical and microbial habitat which influences root growth, respiration, and nutrient exchange. Furthermore, with greater microbial diversity and available nutrients within the community, the plant, in turn, becomes more productive (Sinclair 1824, 
1826). Among the many nutrients present within the communities, nitrogen $(\mathrm{N})$ and phosphorus (P) are the two most essential macro-nutrients. Microbes utilize $\mathrm{N}$ as a nutrient for assimilation, biosynthesis, and an electron donor and acceptor for energy and respiration respectively [143]. Plants use $\mathrm{N}$ and $\mathrm{P}$ as nutrients for many critical roles in their growth and development such as protein synthesis, photosynthesis, adenosine triphosphate (ATP) production, signalling plant metabolic alteration, and converting biochemical reactions. These nutrients exist in many forms throughout the natural ecosystem but are mainly exploited in the form of fertilizers to improve the fertility of soils. In addition, it is noted that roughly half of applied $\mathrm{N}$ to soils is not incorporated by plants but is lost to the environment through leaching and runoff [144]. Real-time monitoring of these nutrients in fabricated micro environments has the potential to allow better understanding of root uptake efficiency, therefore allowing better regulation of fertilizer use, and thereby reducing the environmental impact. Soil analyses for nutrients and microbial estimation have traditionally been accomplished using lab techniques [145]. Although these laboratory techniques for soil analysis are highly accurate, in many cases they cannot be acquired or replicated without damaging the sample. In addition, the techniques are lengthy and time-consuming processes that do not provide the user with real-time monitoring of the dynamic biochemical variations within the rhizosphere and surrounding environments at the pore scale. This poses a challenge to plant phenotype researchers, as plant rhizospheric biochemical concentrations and microbial behaviors can fluctuate at a given time, which in-turn influences plant nutrient uptake mechanisms and metabolism. Plant phenotyping is defined as the comprehensive evaluation of plant physical attributes i.e physiology, growth, development, adaptation, yield, quality, tolerance, resistance, and the measurement of like parameters which aid in the understanding of plant traits [146]4. The conventional methods for plant phenotype analysis require growing seeds and plants in fields, pots, and agar 
dishes [147] [148] [149] [150] [151]5-9. There exists a need for low-cost real-time platforms that can aid researchers in understanding various different phenomena associated with plant germination, growth, metabolism, nutrient uptake, and survival.

Microfluidics and its application in plant science Advances in miniature, low-powered, wireless sensors and microfluidics have shown considerable promise for health/medicine [152]10, diagnostics [153] [154]11,12, energy [155] [156] 13,14, and has now become a growing topic in agriculture as engineered synthetic ecosystems. Microfluidic platforms have been praised for their high throughput, which gives the device the ability to control the flow of microliter fluids and direct them using micrometer channels to specific outputs and chambers, thereby facilitating in situ monitoring of minuscule specimens [157]15. Literature discusses the potential of these devices to aid researchers in uncovering many mysteries and interactions in the soil rhizosphere level, especially in the case of this research, but only recently have microfluidic-based approaches become popular in precision agriculture [158] [159] [160] [161] [162]16-20 Aleklett et al. discuss in detail the various capacities microfluidic devices can be potentially used, these include simulating soil heterogeneity, culturing once unculturable bacteria, manipulating and studying microbial and rhizospheric interactions [163]21. Microfluidics and imaging technologies have demonstrated great potential for advanced plant phenomics. For example, attempts to visualize root growth and cellular behaviors such as host-pathogen interactions were made by [164]22. High throughput imaging has also been implemented with microfluidics to understand plant response to drought, and observe plant root and shoot development [165] [166] [167]23-25. Aside from root imaging using optics, plant-root chemical analysis has been further accomplished using additional laboratory instrumentation such as chemical analyzers. Table 1 describes the application of three microfluidic devices used for plant-root studies; 1) RootChip; 2); RootArray 
and 3) PlantChip. Each device was designed specifically for quantitative monitoring of Arabidopsis thaliana plant phenotypes. The PlantChip device was used to visualize and study root development and pathogenic interaction of Arabidopsis thaliana plants. The Rootchip device demonstrated the ability to monitor growth, galactose, and glucose levels in plants using fluorescent sensors. Phenotype and genotype interactions of Arabidopsis thaliana roots were demonstrated using the RootArray device by [167]25. Current plant microfluidic devices are fabricated using advanced photolithography techniques, which are tedious, time-consuming and expensive processes. These methods utilize excess chemicals and reagents which are costly and require a specialized cleanroom facility that is usually not readily available in microbiological laboratories. The design of these plant-root microfluidic devices can also be tedious, as roots housed in improperly constructed chambers are subject to flooding conditions, often causing asphyxiation in the rhizosphere and leads to plant death. To overcome these barriers, a low-cost facile approach to fabricating sensor integrated germination pod devices for studying rhizosphere micro-environment, root elongation, and nutrient uptake analysis using electrode printing and polymer sheet layering techniques is presented. Further, biochemical tests were carried out to assess the evaluation of plants grown in the micro-sensor germination pods. The functionality of the device was tested by real-time monitoring of a legume nutrient uptake along with plant phenotypic characters.

Plant Chip devices There have been several plant chip reported for studying plant behavior and its interaction with the environment (Table 6.1). However, in this modern age of data processing the integration of nutrient sensors and implementation of wireless communication to transmit the real time data from sensors has been carried. The transfer data can be analyzed using simple machine learning algorithms to generate accurate and predictive models in understanding plant behavior. Real-time wireless monitoring 
can be realized by interfacing the plant sensing structures with any commercially available radio modules, such as a short messaging system (SMS), WI-Fi, ZigBee, Bluetooth, and the most recent technology, LoRa. Currently, the low power, long-range radio module known as LoRa, has become the leading technology for the internet of things (IoT) worldwide. Literature has already demonstrated the potential of these radio modules for real-time monitoring in various disciplines, especially in agriculture where farmers employ these platforms for precision management of resources and crops [37].

\subsection{Materials and Methods}

\subsubsection{Materials}

All the reagents including solutions for electrode preparation and testing were prepared in Millipore distilled water (17.8 mohm). The chemicals and reagents acetone, isopropyl alcohol (IPA), hydrochloric acid $(\mathrm{HCl})$, sulfuric acid, Anthrone reagent, sodium nitrate, di-hydrogen phosphate, pyrrole, agar, and Murashuge Skoog media, were purchased from Sigma Aldrich and were of analytical grade, needing no further treatment. For fabricating the plant pod system, $25 \mathrm{~mm}$ x $75 \mathrm{~mm}$ x $1 \mathrm{~mm}$ glass slides were acquired from Thermo Fisher Scientific, were cleaned with 70 percent IPA, treated with DI water and dried with nitrogen prior to use. Polydimethylsiloxane (PDMS) sheets of approximately $1 \mathrm{~mm}$ thickness were purchased from Interstate Specialty Products (ISP), (Sutton, MA) and were cut and layered, appropriately fitting the device. PDMS sheets present advantages over conventional because it eliminates waste and is cost-efficient when fabricating device on small- and large-scale manufacturing. 


\subsubsection{Methods}

Fabrication of microchip was done by modifying the procedure for developing microreactors in 36. Glass slides were cleaned using IPA, Acetone, and deionized water then air-dried to remove additional impurities, such as grease and smaller foreign particles. The three-electrode configuration was then patterned to glass slides, as further discussed below. The outer edges of the glass substrate were treated using corona plasma torch prior to layering and sandwiching with 2 millimeters thick PDMS sheets between glass slides. PDMS sheets were acquired at the maximum thickness of 1.016 millimeters; therefore, they were also layered by treating with corona plasma discharge and layered to obtain a +2 -millimeter thickness. The thickness can be tailored according to the seed type; therefore, this allowed the sowing of most seeds for plant phenotype studies. Next, inlet and outlet holes were punched into sides of the device for introducing the desired medium and collecting excretion. In this setup to support the growth of plants in the chip, 1 percent agarose along with 1/4 strength MS media (Murashuge Skoog media) [177] was used. The media was incorporated in the chip using a small suction syringe from the top end and bottom end of the device to aid with transport and flow of media.

Fabrication of the sensor Electrodes were fabricated and applied to glass slides using print method. The stencil template for the three-electrode design was created using solid works, then printed onto transferable vinyl adhesive using Silhouette Cameo and Studio software version 4.3.263. The adhesive was attached to a glass slide and smoothed to remove air bubbles. The printed electrodes consisted of layers of Silver/Silver Chloride (60:40) and Carbon Graphene, with the first layer being $\mathrm{Ag} / \mathrm{AgCl}$ and the second layer carbon graphene. After applying the first layer of $\mathrm{AgAg} / \mathrm{Cl}$, the glass received a bake time of 2 hours at 70 degrees $\mathrm{C}$, then allowed to cool dry overnight. The second layer of 

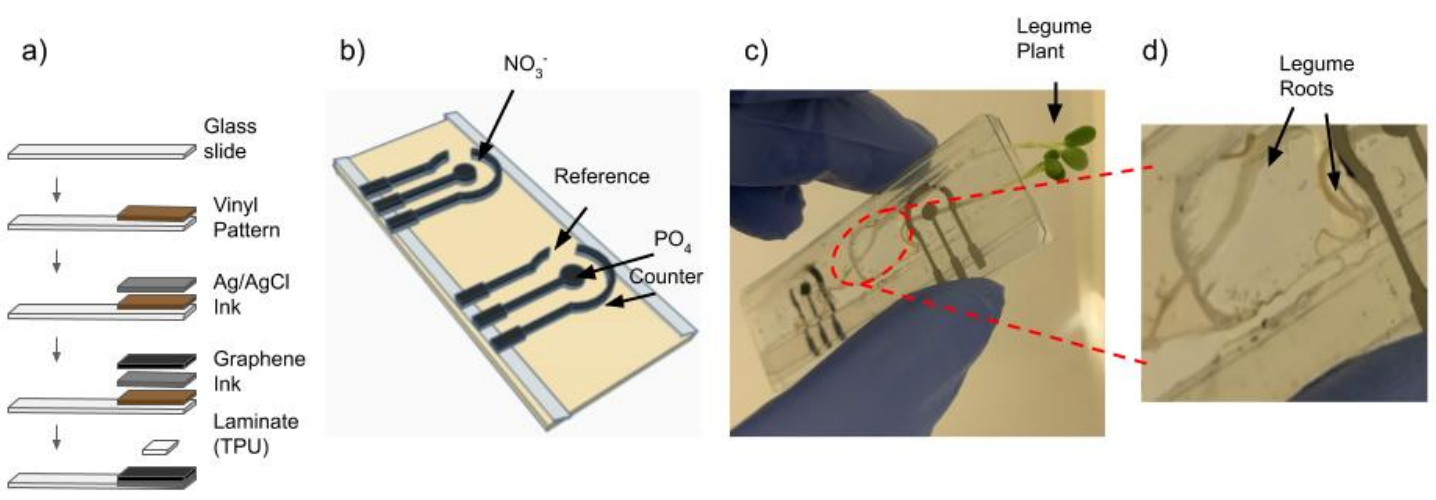

Figure 6.3: a) An illustration of the fabrication process; b) illustration of germination wells with integrated sensors and PDMS sheets; c) view of the sensor integrated germination pod device facilitating the growth of the legume plant; d) zoomed view of legume roots housed in sensor pod device.

carbon graphene was applied similarly using ink print method then dried at 65 degrees for 1 hour. After drying, the validity of the fabricated device was tested using cyclic voltammetry of $5 \mathrm{mM}$ ferriferous solution (-1V TO $1 \mathrm{~V})$. Nitrate and phosphate doped polymer sensors were applied using electrochemical methods. The 1 to 1 ratio of $1 \mathrm{~mol}$ of pyrrole and $.5 \mathrm{~mol}$ of nitrate were mixed in a 5-millimeter vial, where the solution was purged with nitrogen for 15 minutes to displace oxygen. Pyrrole is photosensitive and can be contaminated with oxygen, therefore it was stored in light-sensitive vials and refrigerated. The doped polymer membranes were prepared using electrochemical methods. A constant current of 100-150 microamps was applied for fifteen minutes using a PGSTAT. After selective sensor fabrication, devices were conditioned in 100 millimolar NaNO3 tubes for 24 hours prior to use.

The phosphate sensors were also prepared in a similar manner under the same condi- 


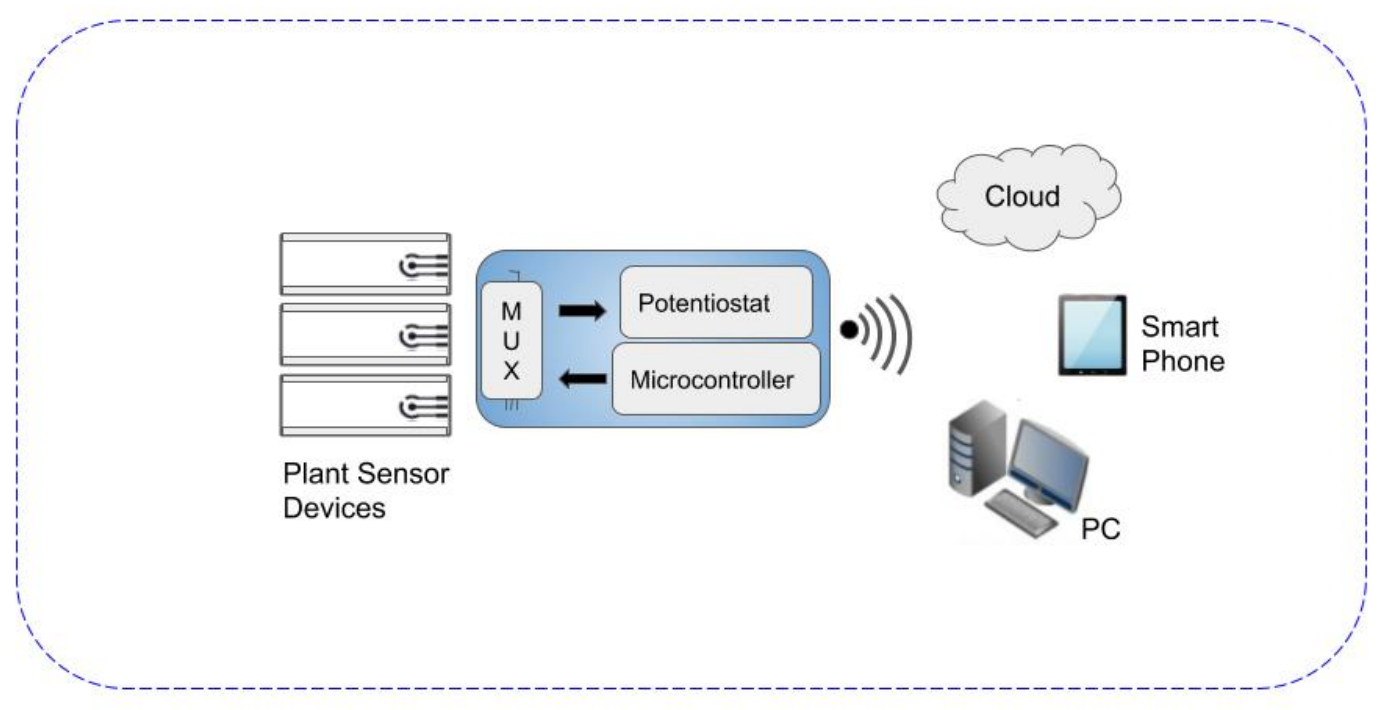

Figure 6.4: Flow diagram illustrating the multiplexed plant sensing devices communication to cloud, smartphone, and personal computer

tions stated above, utilizing a 1-to-1 ration of pyrrole and $.5 \mathrm{~mol}$ of di-hydrogen phosphate. The solutions were mixed into a $5 \mathrm{~mL}$ vial and purged with nitrogen gas. A constant current of 100 microamps was applied for ten to fifteen minutes using a PGSTAT. The devices were stored in a $100 \mathrm{mM}$ solution of dihydrogen phosphate for 24 hours prior to use.

Wireless monitoring of plant nutrient uptake Real-time wireless monitoring of fabricated sensor devices can be realized when interfacing any commercially available radio modules, such as a short messaging system (SMS), WI-Fi, ZigBee, Bluetooth, and the most recent technology, LoRa. In this paper, a real-time monitoring platform has been employed for monitoring plant nutrient uptake of nitrate and phosphate. The microcontroller was interfaced with the LoRa radio module and dipole antenna to facilitate communication to a cloud platform. 


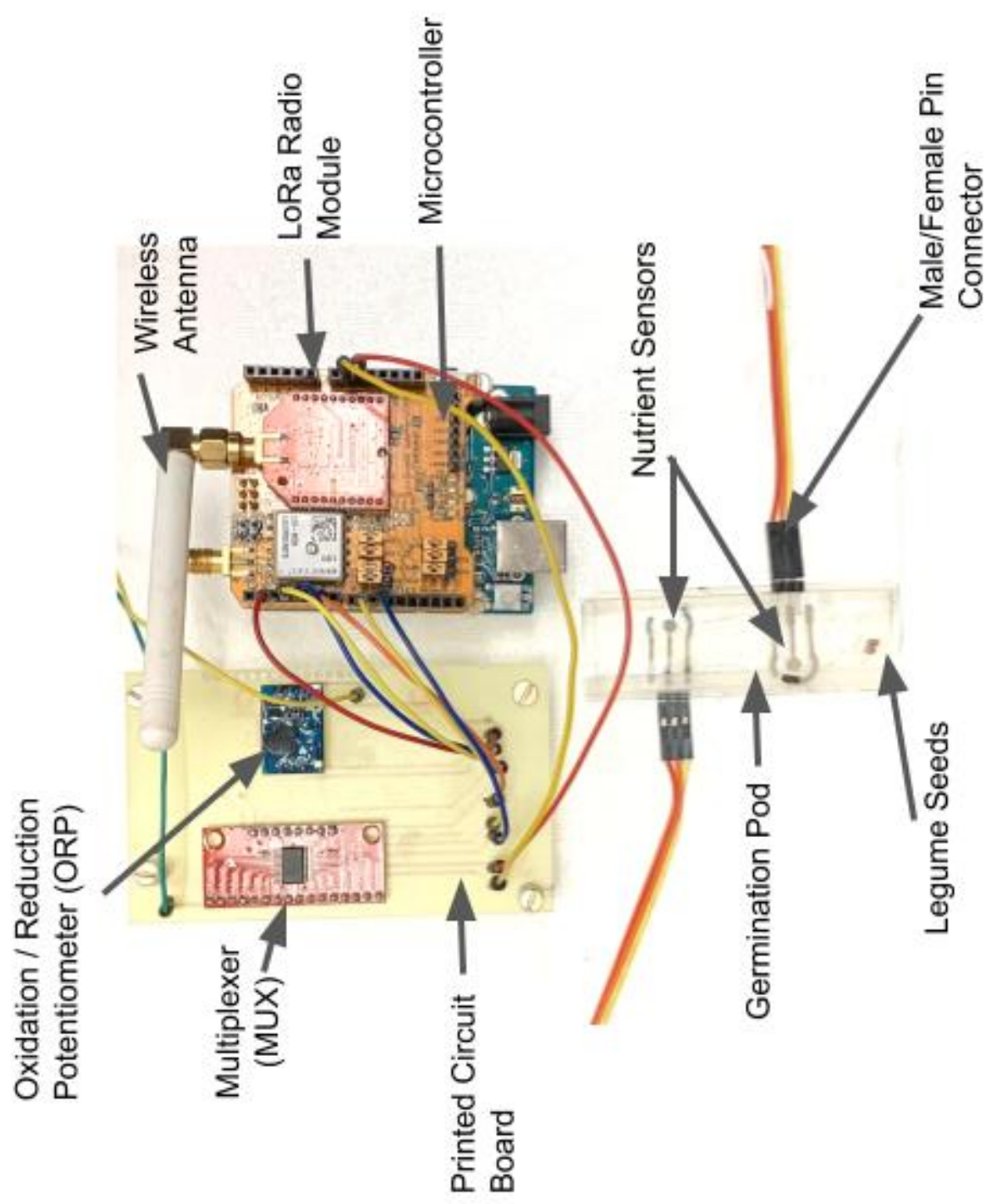

ฮี

帘

- 00

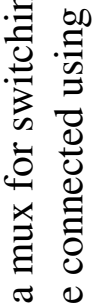

ฮี

巻

웡

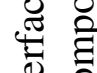

.

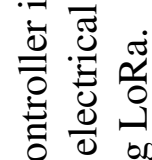

仓

-

๘

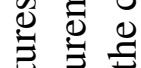

芯

을

उ

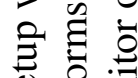

Ð

. ¿

:

(3)

ป

긍

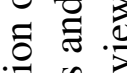

创

总 坖

iุ ठั.

0 ह

总 壳 


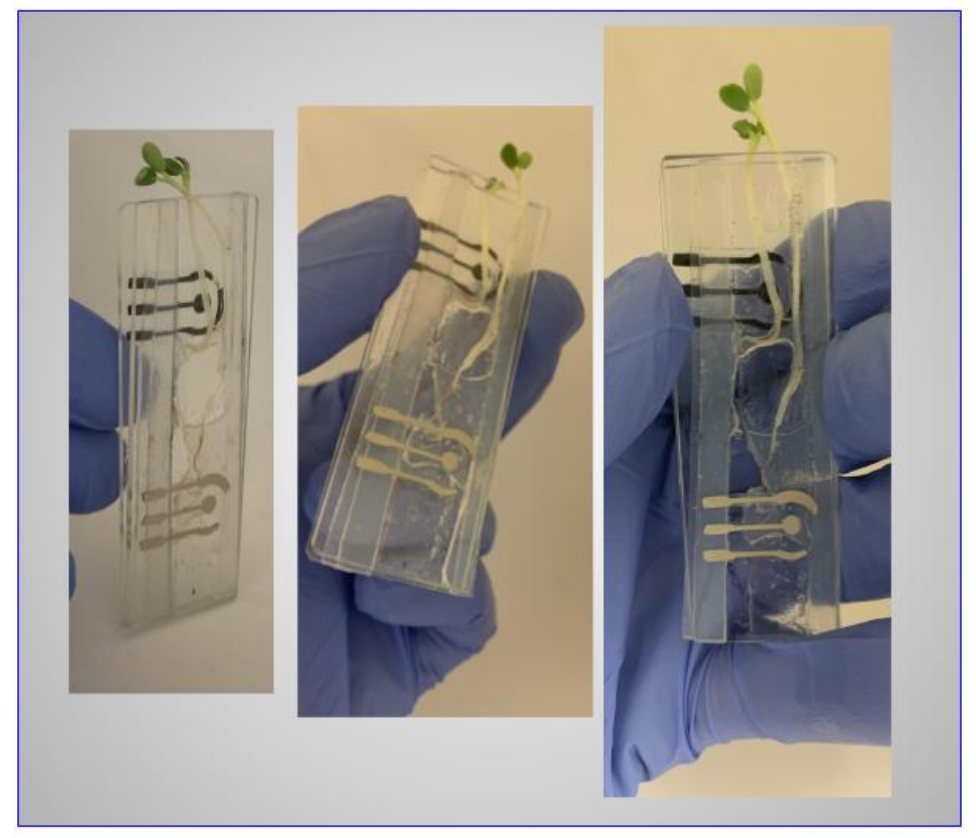

Figure 6.6: Images of pod with integrated printed micro sensors and legume plants.

\subsubsection{On-chip plant phenotype analysis}

Seed germination Crimson Red Clover was chosen because of its relatively small seed size, considerable growth in a short time period, and are leguminous crops of agricultural importance. Legume crops are popular among companion farming intercropping operations, and farm crop rotations due to this phenomenon because biodiversity is added to the soil along with reduced expenses and inputs for increased growth and yield. The experimental design consisted of two treatment groups each containing ten seeds: a treatment group containing ten conventionally grown seeds, and treatment with ten seeds grown in pods. In the case of the conventional grow process, seeds were placed on biostrate fabric then moistened for germination. After 24 hours, root/shoot development was observed. In the case of the sensor pods, seeds were planted and continuously monitored as nutrient uptake efficiency was measured. 
Shoot and root analysis Observation and analysis of root and shoots typically require the removal of the plant from its natural state and use of methods which often damages the sample. The developed sensor pods enabled root shoot analysis without removing or damaging the sample. Since the plant pod is transparent therefore the root measurement can be easily be done by observing the pod under a stereo-microscope without disturbing its microenvironment. The plants were grown for a period of 7 days and were continuously monitored [178]. The seven-day root and shoot height were recorded, then the averages of the treatment groups were derived at day seven.

Estimation of total Chlorophyll Chlorophyll and nitrogen are two of the most vital components of the plant. During photosynthesis, chlorophyll functions to absorb energy from the sun and transform into chemical energy for the plant. The chemical structure of each chlorophyll molecule contains a porphyrin (tetrapyrrole) nucleus with a chelated magnesium atom at the center and a long-chain hydrocarbon (phytyl) side chain attached through a carboxylic acid group [179]. In this method, Chlorophyll is extracted in 80 percent acetone and the absorption peaks at $663 \mathrm{~nm}$ and $645 \mathrm{~nm}$ are read in a spectrophotometer. First, the sample is weighed in a clean mortar. The sample was then ground into a pulp with the addition of $2 \mathrm{~mL}$ of 80 percent acetone. Next, the sample was centrifuged at 5,000 rpm for five minutes and the supernatant was transferred into a $1.5 \mathrm{ml}$ Eppendorf tube. The absorbance of the solution was then read at $645 \mathrm{~nm}, 663 \mathrm{~nm}$ and $652 \mathrm{~nm}$ against the solvent (80 percent acetone) blank. The amount of chlorophyll present in the extract (mg chlorophyll per $\mathrm{g}$ tissue) were then calculated using additional equations:

$$
\begin{aligned}
& \text { mgchlorophylla/gtissue }=12.7(A 663)-2.69(A 645) \mathrm{V} / 1000 \mathrm{~W} \\
& \text { mgchlorophyllb/gtissue }=22.9(A 645)-4.68(A 663) \mathrm{V} / 1000 \mathrm{~W}
\end{aligned}
$$




$$
\text { mgtotalchlorophyll } / \text { gtissue }=20.2(A 645)+8.02(A 663) \mathrm{V} / 1000 \mathrm{~W}
$$

Where $\mathrm{a}$ is the absorbance at specific wavelengths, $\mathrm{V}$ is the final volume of chlorophyll extract in $80 \%$ acetone and $\mathrm{W}$, is the fresh weight of tissue extracted. In addition, the soil-plant analysis data (SPADTM) was used to count the number of chlorophyll present within each section of the legume leaf and the average was determined in each treatment group (table 6.2). [180]

Estimation of total Nitrogen The total nitrogen analysis was made using the wet oxidation digestion method. The sample tissue was weighed and placed in pre-cleaned and autoclaved borosilicate tubes and rinsed with $0.1 \mathrm{~N} \mathrm{HCl} .2 \mathrm{ml}$ of concentrated sulfuric acid was added and the tubes were kept at 300 degrees $\mathrm{C}$ for 2 hrs. 5-8 drops of 30 percent hydrogen peroxide were added and incubated again at 300 degrees $\mathrm{C}$ for $10 \mathrm{~min}$. After the extract appeared colorless, it was then topped with $25 \mathrm{~mL}$ of deionized water and used for elemental analysis. Total $\mathrm{N}$ analysis was done by Vespodest Gerhard [180].

Estimation of total carbohydrates by Anthrone method Carbohydrates are first hydrolyzed into simple sugars using dilute hydrochloric acid. In hot acidic medium, glucose is dehydrated to hydroxymethylfurfural. This compound forms with Anthrone a red to a green-colored product with an absorption maximum at $630 \mathrm{~nm}$ [181]. In this procedure, the sample is weighed and minced in a $5 \mathrm{~mL}$ tube of $\mathrm{Na}-\mathrm{K}$ buffer. The supernatant and 0.5 and $1 \mathrm{~mL}$ aliquots are taken for analysis. The standards are prepared by taking 0 , $0.2,0.4,0.6,0.8$ and $1 \mathrm{~mL}$ of the working standard. 0 serves as blank. $4 \mathrm{~mL}$ of Anthrone reagent was added and heated in a boiling water bath for 8 minutes. The sample was allowed to cool rapidly and the green to dark green color was read at $630 \mathrm{~nm}$. A standard graph was drawn by plotting the concentration of the standard on the X-axis versus 
absorbance on the Y-axis. From the graph, the amount of carbohydrate present in the sample tube was calculated using additional equations:

$$
\text { Carbsper } 100 m g o f \text { sample }=(\text { mgofglucose }) /(\text { volumeoftestsample }) 100
$$

\subsection{Summary}

This chapter explores the fabrication of printed sensor sheets and printed sensor pods integrated with a kind of wireless potentiometry for in-situ $\mathrm{N}$ monitoring in field setting. This chapter reported a disposable IoT gardening soil sheet, capable of analyzing real-time soil nitrate concentration during leaching and irrigation events, and the wireless SensePod system, which is capable of remote monitoring plant growth and root nutrient uptake. For the Sensor sheet, the nitrate doped polypyrrole ion selective electrode (N-doped PPy ISE) sensor array featured a fault tolerant circuit design multiplexed to an oxidation and reduction potentiometer that can rapidly detect nitrate levels in soil leachates. Measurement data are transmitted via Waspmote ZB Pro SMA 5dBi radio, 6600mAh rechargeable battery, 7.4-volt solar panel, and a Meshlium ZigBee PRO access point to cloud server and mobile device. Gold electrodes were also fabricated and utilized as a measurement standard. 


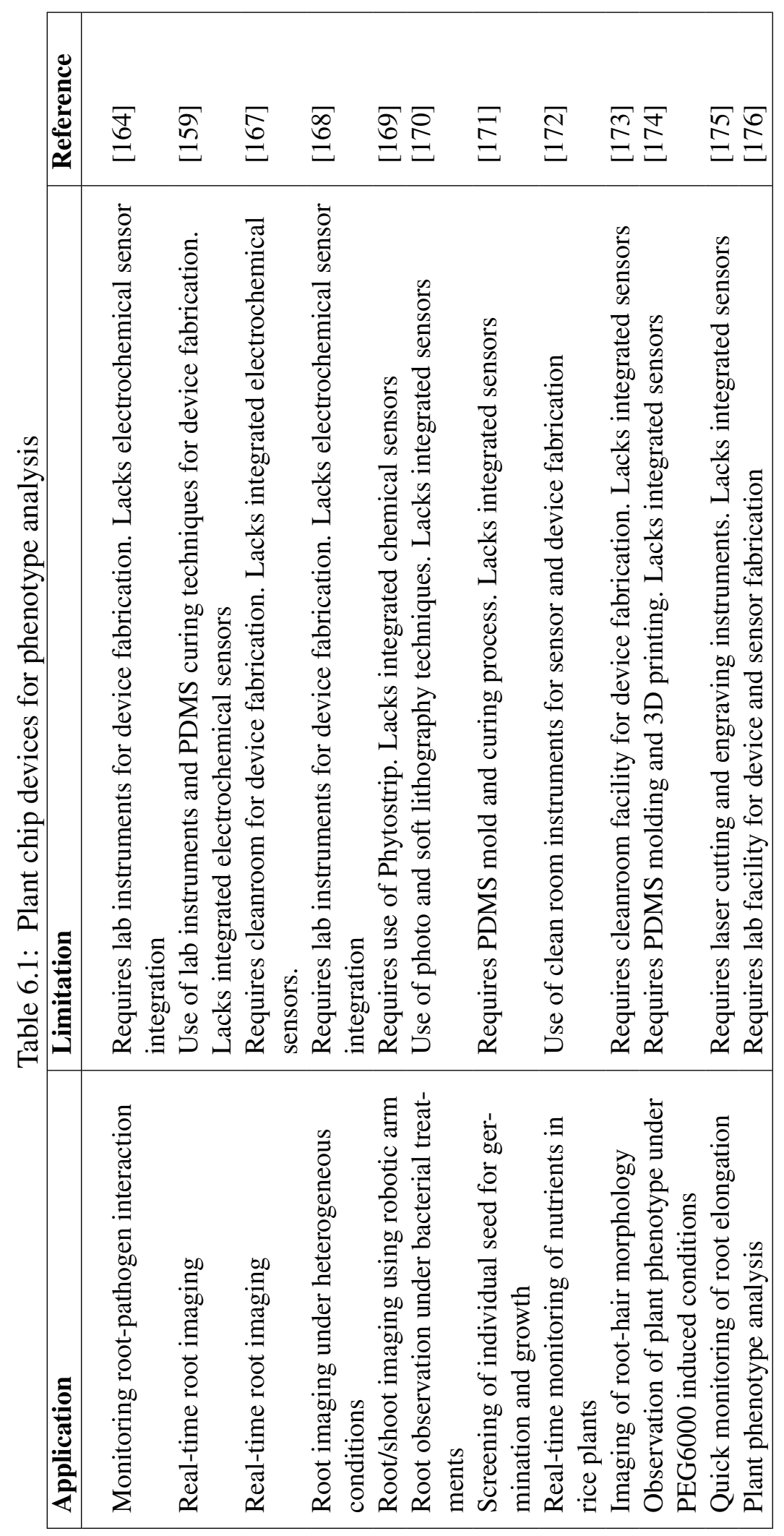




\section{CHAPTER 7}

\section{RESULTS AND DISCUSSIONS}

\subsection{Overview}

This chapter reports a disposable IoT gardening soil sensor sheets and pods. The sensor sheets are capable of analyzing real-time soil nitrate concentration during leaching and irrigation events. The nitrate doped polypyrrole ion selective electrode $(\mathrm{N}$-doped PPy ISE) sensor array sheet features a fault tolerant circuit design multiplexed to an oxidation and reduction potentiometer that can rapidly detect nitrate levels in soil leachates. Measurement data are transmitted via Waspmote ZB Pro SMA 5dBi radio, 6600mAh rechargeable battery, 7.4-volt solar panel, and a Meshlium ZigBee PRO access point to cloud server and mobile device. This chapter investigates the gardening IoT sheets as a viable tool for in situ nitrate mapping, and to potentially help everyday home and commercial gardeners reduce excessive fertilizer application.

\subsection{Sensor Sheets Results and Discussion}

\subsubsection{Sensor Sheets}

\section{Results and Discussion}

Electrodes were inkjet printed on a paper substrate. Working electrodes were modified for nitrate sensitivity by electrodeposition of pyrrole. As a result, a thin film polymer membrane of N-doped pyrrole was formed capable of soil nitrate measurements. A prototype sheet that comprises of 8 electrodes in a $5 \times 3$ inch area was developed (Figs.

6.1a, 6.1b). Working electrodes of the individual sensors are coupled to a custom potentiostat shown in a previous chapter (Fig. 6.2) via a TCA9548A 1-to-8 I2C multiplexer 
module using a flex connector. Electronic sensing is enabled via a microcontroller and an IoT network. Individual electrodes are engaged by the potentiostat for a certain duration by varying the control signals and time delay appropriately. Nitrate sensitivity, detection range and the stability of the electrodes were found to have an influence on the properties of electrodeposited sensors. A constant current applied for a duration of 5-20 minutes yielded nitrate sensitive working electrodes. Pre-treatment of electrodes in 10 $\mathrm{mM} \mathrm{KOH}$ solution improved the hydrophilicity of substrates, which also helped in forming a uniform electrodeposited layer. Electrodeposited electrode sheets were incubated at 70C for $15 \mathrm{~min}$ for enhanced stability.

Figs. 7.1a, 7.1b shows circular grains around 50-200 nm in diameter for 5 and 10 minute (E5 and E10) long electrodeposition. At longer electrodeposition times, larger clusters were observed (Figs. 7.1c-7.1d). The non-uniformity and nonconductive nature of the larger clusters were found to hinder the ionic exchange during electrochemical measurements. Linearity and sensing range was found to be affected by the duration of electrodeposition. Electrodes deposited for $15 \min (\mathrm{E} 15)$ were found to have a linear response in the 5 ppm to 90 ppm nitrate levels. Fig. 7.2a shows the differential pulse voltammetry (DPV) current response of sensors to varying nitrate levels. Nitrate sensitivity of sensors that were electrodeposited at varying times is depicted in Fig. 7.2b. As the films grew thicker, the slope of the nitrate response curve was found to be higher nearing a Nernstian response. Apparent from the sensor response, the electrodes that were electrodeposited for a duration of 15 minutes was found to have the best linear sensitivity as compared to the other electrodes. Apparently from the sensor response slopes, electrode E15 was found to be more than $110 \%$ sensitive to nitrate levels as compared to E5 and E10. However, electrode E20 was found to be the least sensitive among all the electrodes, despite having a larger area. It was assumed that the non-uniformity and nonconductive nature of the larger clusters are hindering the ionic exchange during elec- 

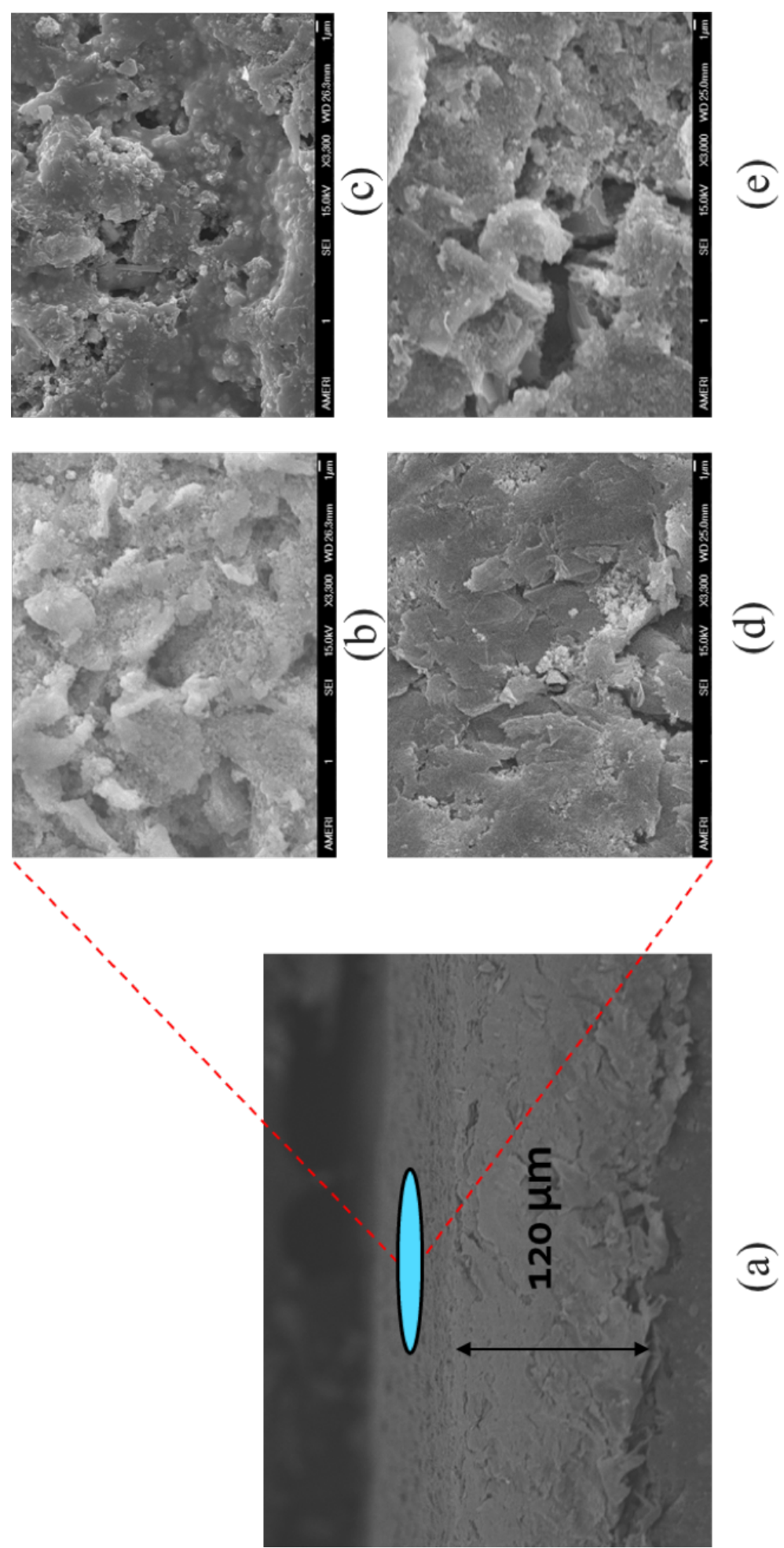

$\overparen{\nabla}$

Figure 7.1: SEM micrographs of (a) cross section of the electropolymerized working electrodes. Electrodeposition of pyrrole for varying time durations (b) 5 (c) 10 (d) 15 (e) 20 minutes at $100 \mu \mathrm{A}$ 

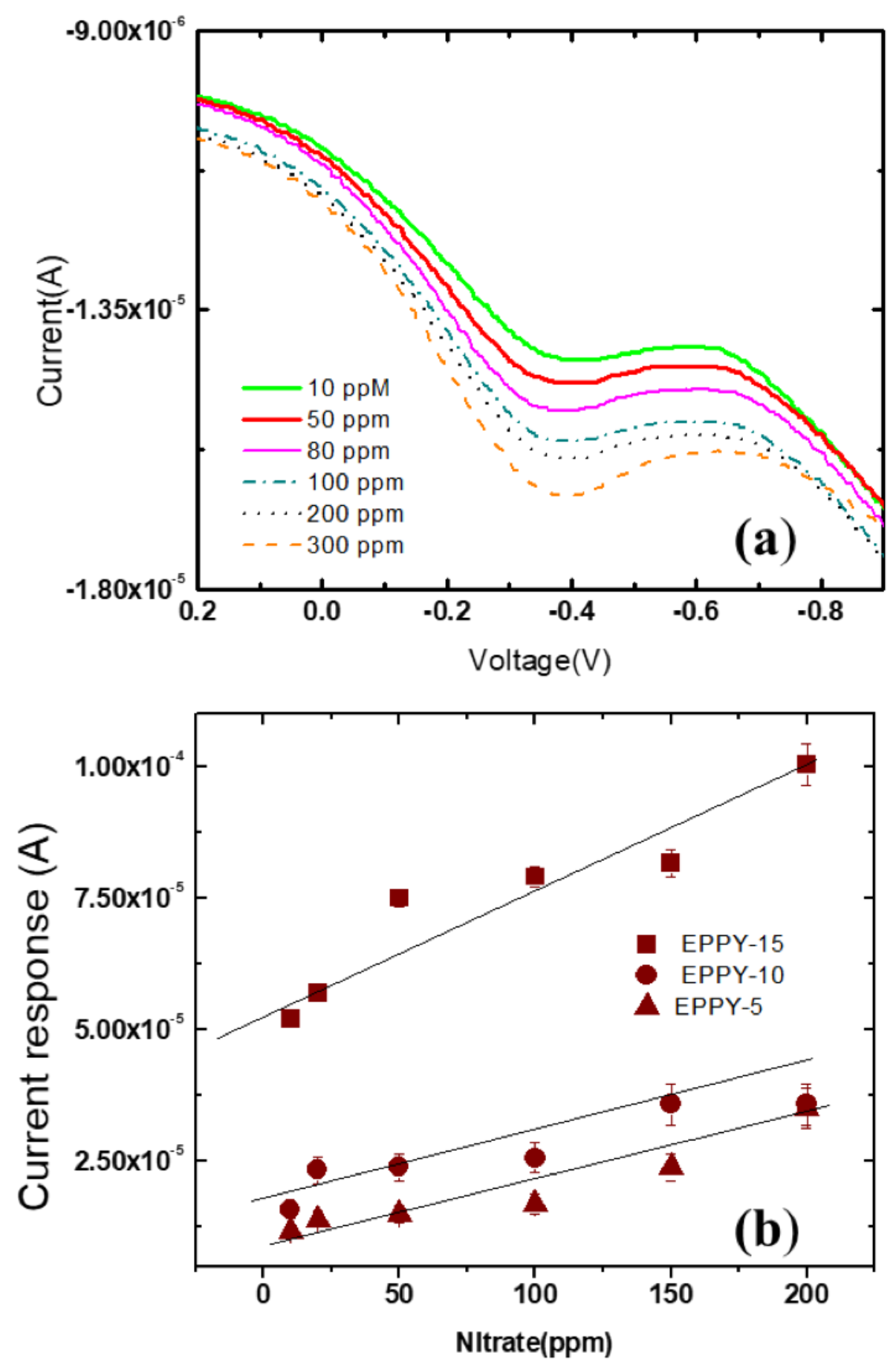

Figure 7.2: Soil leachate studies: (a) Differential pule voltammetry (DPV) response of sensors to varying nitrate levels (b) Current response of E5,E10, and E15 to nitrate levels (10-200 ppm) 

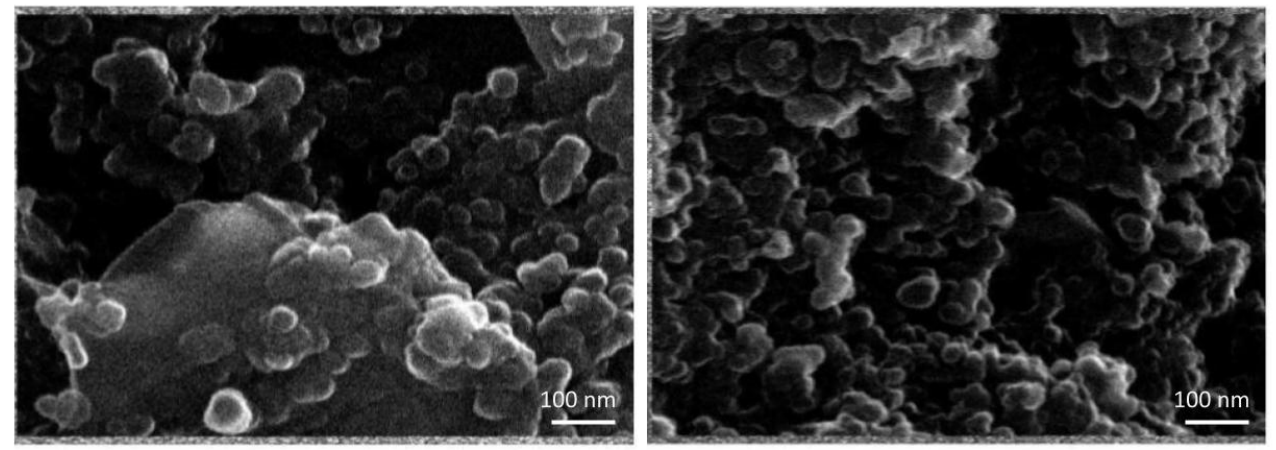

Figure 7.3: Illustration of Au nanoparticles deposited on Carbon electrode.

trochemical measurements.

Carbon doped Au-NP electrode The Figure 7.3 illustrates an SEM image of the surface area carbon electrodes. The carbon electrode surface contains nano-particles ranging in size from 50-60 nanometers in diameter which had been deposited by way of drop-cast deposition. SEM also revealed the surface morphology of the carbon electrodes to the rough and uneven surface area of the Au-NP carbon electrode. This includes uneven distributions of AU nano-particles, often times in scattered clusters, observed throughout the surface of the carbon electrode. It was assumed that this also accounted for the decreases in nitrate sensor sensitivity when compared to the Au doped Au-NP electrodes (shown in Figure 7.4).

Au doped Au-NP electrode The Figure 7.4 illustrates the Au electrode which had been deposited with Au nanoparticles by way drop-cast method. Unlike the Carbon based electrodes, the surface of the Au electrode revealed to be much smoother and free of rough-jagged areas. It was assumed that the smoother surface facilitated a more ho- 


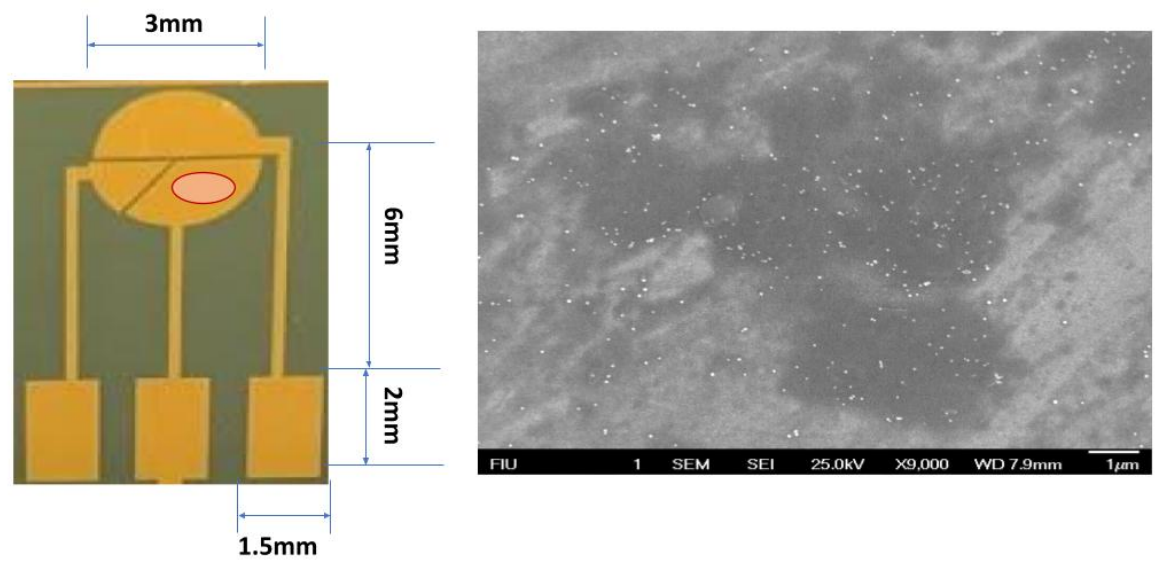

Figure 7.4: Illustration of Au electrode fabricated in clean-room (left) and an SEM image of Au electrode with Au nano-particles (right).

mogeneous dispersion of nanoparticles on top of the Au electrode. This also led to better sensor sensitivity and stability when compared to the Carbon doped Au-NP electrodes.

Monitoring nitrate leachate Fig. 7.5 depicts the amount of leached nitrate as detected by the sensor. Three different soil types of limestone-derived udorthents with known carbon and nitrogen contents (Table 7.2) were used for this study. Sensors were installed one inch beneath the top of the soil beds. Readings were taken 600 seconds after irrigation allowing time for leachate to come in contact with the sensor. Soil samples, spiked with $100 \mathrm{mM}$ nitrate solution, were used for leaching studies. Irrigation was applied at a fixed daily rate and the leachate samples from the bottom drain were analyzed for six consecutive irrigation cycles. The response of the sensor to varying irrigation cycles reflected the leaching rates. Leaching trends were found to be very similar for all three soil samples. Leaching was found to be highest for the first 2 irrigation cycles. This can be due to loosely bound nitrate. Depending on the nitrate leach rate, the sensor 
Table 7.1: Comparison of $\mathrm{NO}_{3}^{-}$Sensors

\begin{tabular}{|c|c|c|c|c|c|}
\hline $\begin{array}{l}\text { Electrode } \\
\text { Type }\end{array}$ & $\begin{array}{l}\text { Linear Range } \\
(\mathrm{mol} / \mathrm{L})\end{array}$ & Slope & $\begin{array}{l}\text { Limit of Detec- } \\
\text { tion }\end{array}$ & $\begin{array}{l}\text { Response } \\
(\mathrm{sec})\end{array}$ & Ref. \\
\hline Carbon & $1.8 \mathrm{E}^{-5}-2.2 \mathrm{E}^{-2}$ & $\begin{array}{l}-54.9+/- \\
1.4\end{array}$ & $6.32 \mathrm{E}^{-6}$ & $15-19$ & $\begin{array}{l}\text { This } \\
\text { work }\end{array}$ \\
\hline $\begin{array}{l}\text { Carbon w/ } \\
\mathrm{NP}\end{array}$ & $10^{-6}-.5 \mathrm{E}^{-2}$ & $-57.2+/-.7$ & $1.33 \mathrm{E}^{-6}$ & $9-13$ & $\begin{array}{l}\text { This } \\
\text { work }\end{array}$ \\
\hline Bare $\mathrm{Au}$ & $8 \mathrm{E}^{-4}-1 \mathrm{E}^{-1}$ & $\begin{array}{l}-59.1+/- \\
1.7\end{array}$ & $4.92 \mathrm{E}^{-6}$ & $<9$ & $\begin{array}{l}\text { This } \\
\text { work }\end{array}$ \\
\hline AuNP's & $1 \mathrm{E}^{-6}-1 \mathrm{E}^{-1}$ & $\begin{array}{l}-58.8+/- \\
1.1\end{array}$ & $1.14 \mathrm{E}^{-6}$ & $<7-10$ & $\begin{array}{l}\text { This } \\
\text { work }\end{array}$ \\
\hline $\mathrm{Ag} / \mathrm{AgCl}$ & $3.8 \mathrm{E}^{-6}-2.2 \mathrm{E}^{-2}$ & $\begin{array}{l}-58.6+/- \\
1.7\end{array}$ & $1.78 \mathrm{E}^{-6}$ & $7-10$ & 176 \\
\hline
\end{tabular}

Table 7.2: Nitrate leachate levels of three lime stone-derived udorthents soil samples during irrigation cycle (LOW: 5-40 ppm, MED: 40-100 ppm, HIGH: 100-300 ppm) as predicted by the IoT soil sensor network

\begin{tabular}{lllllll}
\hline Soil sample & 1 & 2 & 3 & 4 & 5 & 6 \\
\hline 1 & HIGH & HIGH & MED & LOW & LOW & LOW \\
2 & HIGH & HIGH & MED & LOW & LOW & LOW \\
3 & HIGH & HIGH & MED & LOW & LOW & LOW
\end{tabular}

response has been categorized into three; LOW: 5- 40 ppm, MED: 40-100 ppm, HIGH: 100-300 ppm . When the response of the sensor is HIGH for two consecutive times, an alert signal is sent out by an external micro-controller to the IoT network. However, stability of the sensor was found to be degrade after 3 consecutive tests. This can be due to the deterioration of the pyrrole surface from the mud particulates in the leachate.

Waspmote Agriculture Sensor Board Pro serves as the microcontroller and the IoT network. Data packets were transmitted via a Waspmote ZB Pro SMA 5dBi radio, 6600mAh rechargeable battery, 7.4-volt solar panel, and a Meshlium ZigBee PRO access point. Data obtained from individual sensors were directed to Meshlium access point and stored directly to the hard drive or sent to a cloud service. Meshlium is a Linux router which works as the gateway to the waspmote sensor network. Inserting a sim card onto the 


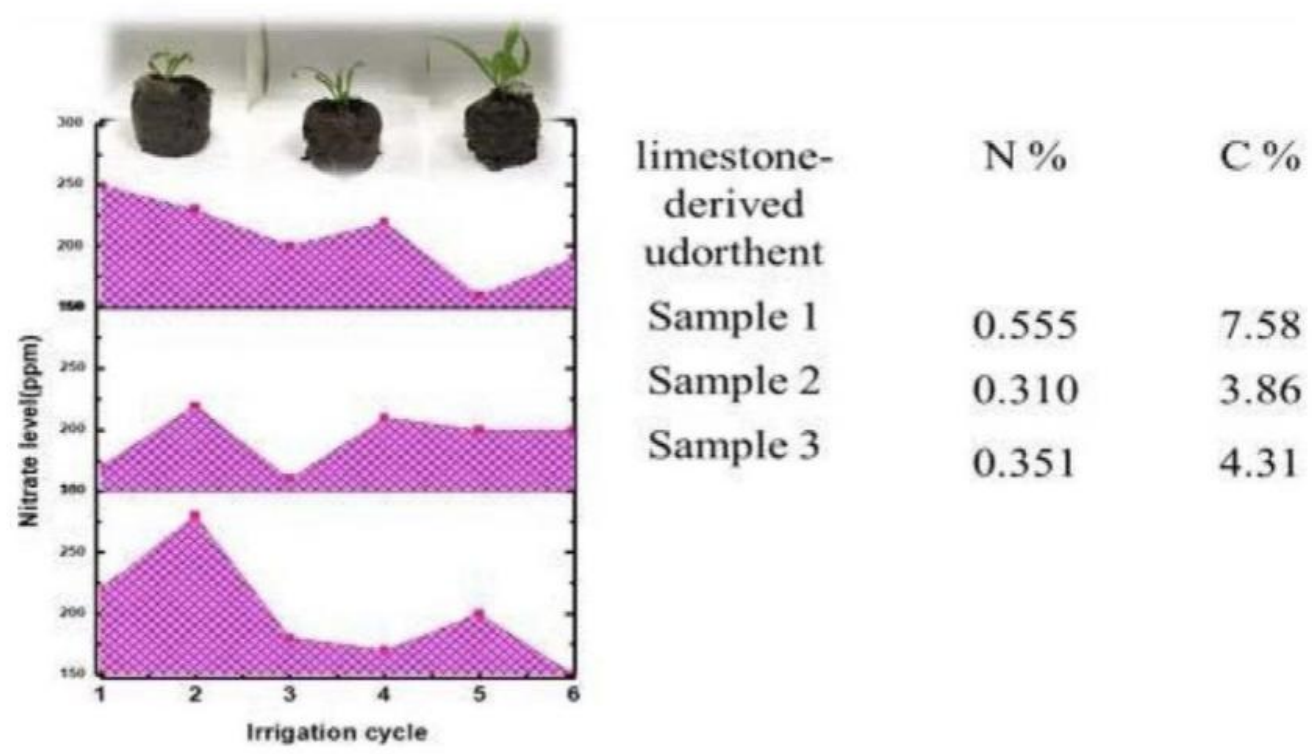

Figure 7.5: Nitrate leaching in limestone-derived udorthent soil samples

waspmote sim slot allows for data and commands to be transmitted to cellular devices. A ZigBee radio transmit data frames to the meshlium, which operates at $2.54 \mathrm{Ghz}$, using a transmission power of $50 \mathrm{~mW}$ and a line of sight $5 \mathrm{dBi}$ dipole antenna to cover a range of 7000 meters. Out of three soil samples tested, all of them showed HIGH leachate levels consecutively for two irrigation cycles. A leaching rate $i, 100 \mathrm{ppm}$ of leaching for two consecutive cycles (Cycle 1 and 2). After Cycle 3, the leachate level was found to drop consistently. Similar trend was observed with samples 2 and 3. Hence, re-fertilization of the soil will be based on the crucial information from the developed soil sheet sensor.

\subsubsection{Sensor Sheets Summary}

This chapter highlighted the development of an IoT enabled soil sensor sheet sensor capable of electrochemically detecting nitrate leachates. The sensor sheet was inkjet printed on a paper substrate and modified via electrodeposition. Initial studies indicate 
that the analytical current response is linearly proportional to soil leachate nitrate content, however, the stability of the sensor was found to be degrade after 3 consecutive tests. This can be due to the deterioration of the pyrrole surface from the mud particulates in the leachate. Sensor deterioration can be due to various reasons, such as concentration of soil nutrients, $\mathrm{pH}$, ionic composition, non-specific adsorption on the surface of the electrode, weather conditions, and soil microbial interaction. Further research seeks to address the sensor abnormalities as observed from the previous experiments. The potential of the developed wireless sensing platform was demonstrated by detecting the soil leachate levels in three samples of limestone-derived udorthents with respect to irrigation cycles. Measurement data are transmitted via Waspmote ZB Pro SMA 5dBi radio and a Meshlium ZigBee PRO access point to cloud server and mobile device

\subsection{SensePod Results and Discussion}

\subsubsection{SensePod}

\section{Results and Discussion}

Characterization of sensor The device was prepared in house using cost-effective methods that are adaptable for large scale manufacturing. Prior to electropolymerization, the vitality of the electrodes was tested in a ferrous solution. Figure 7.6 shows CV of electrodes prepared using print methods. The working and counter electrode consisted of printed Gr and the supporting reference electrode featured an additional layer of $\mathrm{Ag} / \mathrm{AgCl}$. After device fabrication, they were electrochemically characterized using prepared ferrous solutions of $1 \mathrm{mM}, 2 \mathrm{mM}$ and $5 \mathrm{mM}$ (Figure 7.6). 


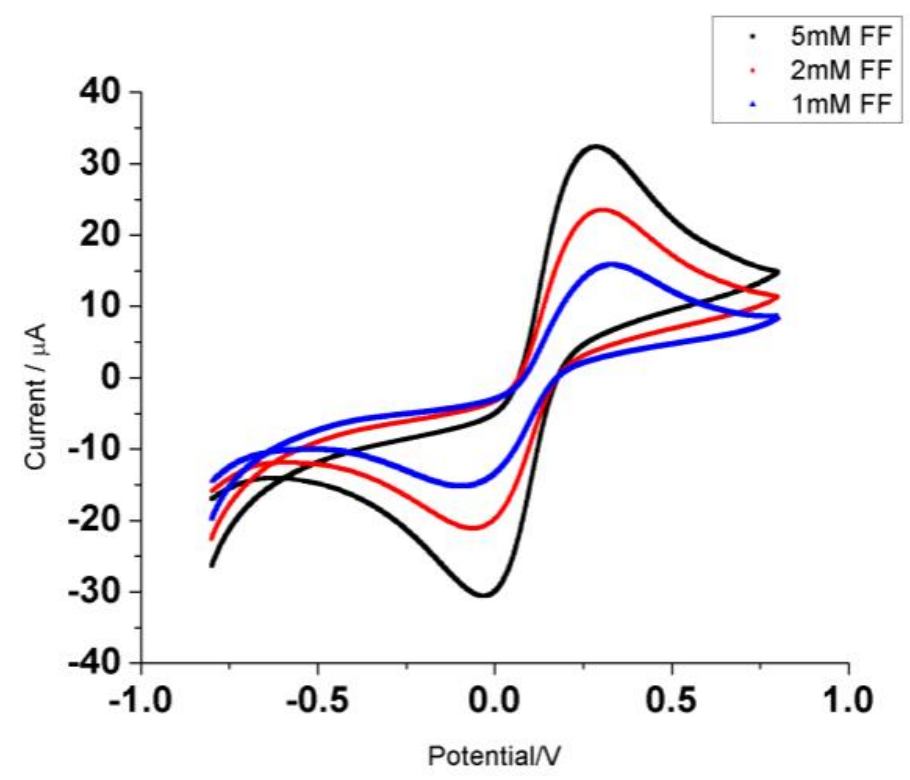

Figure 7.6: Cyclic voltammogram of fabricated sensors, $\mathrm{Gr}-\mathrm{Ag} / \mathrm{AgCl}$ silver in $1 \mathrm{mM}$, $2 \mathrm{mM}$, and 5 millimolar ferrous solutions.

Voltammetric response of doped PPy sensors Figure 7.7 shows the cyclic voltammograms of doped PPy sensors between voltage ranges of $-1 \mathrm{~V}$ and $1 \mathrm{~V}$ at $1 \mathrm{mM}$ concentration of NaNO3- and $\mathrm{H} 2 \mathrm{PO} 4$ at a scan rate of $20 \mathrm{mV} / \mathrm{s}$. The graphs demonstrate the sensors to be electroactive to the concentrations with reduction peaks at approximately $-0.2 \mathrm{~V}$ and $-0.4 \mathrm{~V}$ respectively. Subsequently, the voltage at the reduction peak was chosen to deliberate the calibration test with nitrate solutions over a span of $1 \mathrm{mi}$ cromole to 1 millimolar. The in house sensor performed comparably to commercial ionselective electrodes.

Calibration plots of doped PPy sensors The response of the doped sensors was tested for nitrate and phosphate ions in respective calibration solutions. Figure 7.8 shows the calibration plot for nitrate and phosphate, created by extrapolating the peak currents for each concentration. The linear fit to the nitrate (Eq.7.1) and phosphate (Eq.7.2) 

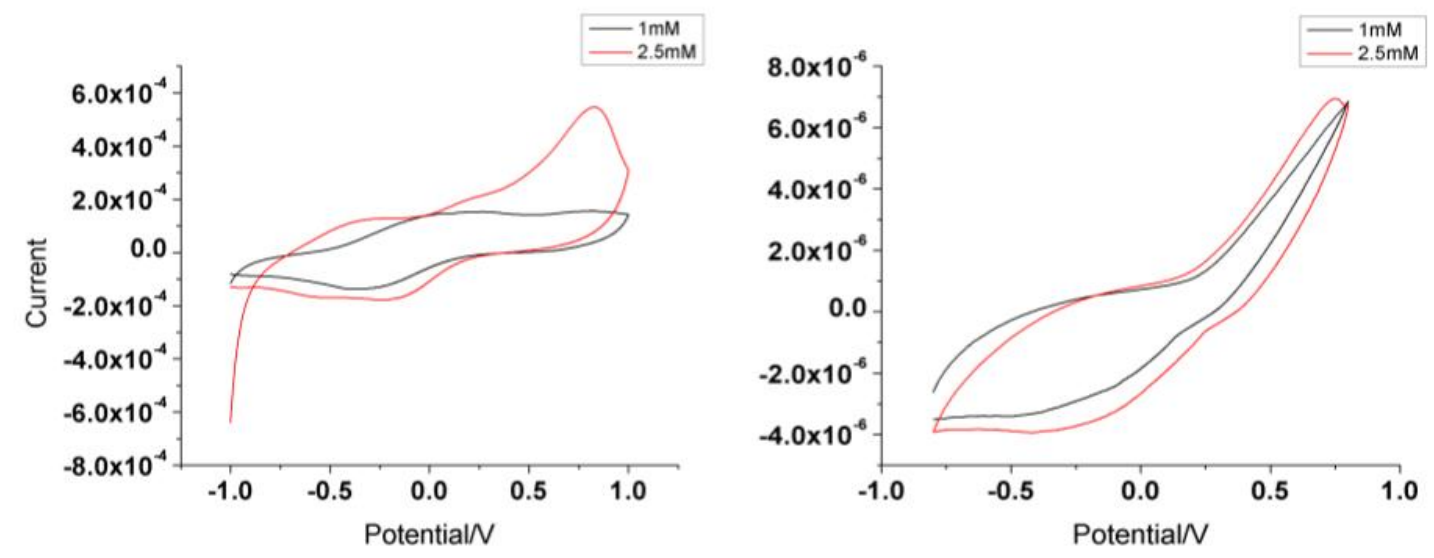

Figure 7.7: Cyclic voltammogram of the doped PPy sensors in $1 \mathrm{mM}$ of NaNO3- and $\mathrm{H} 2 \mathrm{PO} 4$ electrolyte solution at a scan rate of $25 \mathrm{mV} / \mathrm{s}$

forms are as follows:

$$
\begin{gathered}
y=1.74 E^{-05}(\text { conc. } \mu m o l)+9.75 E^{-05} \\
y=4.41 E-07(\text { conc. } \mu m o l)+5.57 E^{-07}
\end{gathered}
$$

Sensing In situ uptake of plant nutrients The doped PPy sensors were employed to measure phosphate and nitrate within the growth medium over a span of seven days (Figure 7.9). As a result of the nutrient uptake, the microreactor root chamber experienced a nutrient recharge, where nutrients were replenished after 5 days over a period of 7 days. The plant uptake of nitrate was greater than phosphate. It was postulated that this may be due to a larger amount of $\mathrm{N}$ required during the seedling growth stage. 

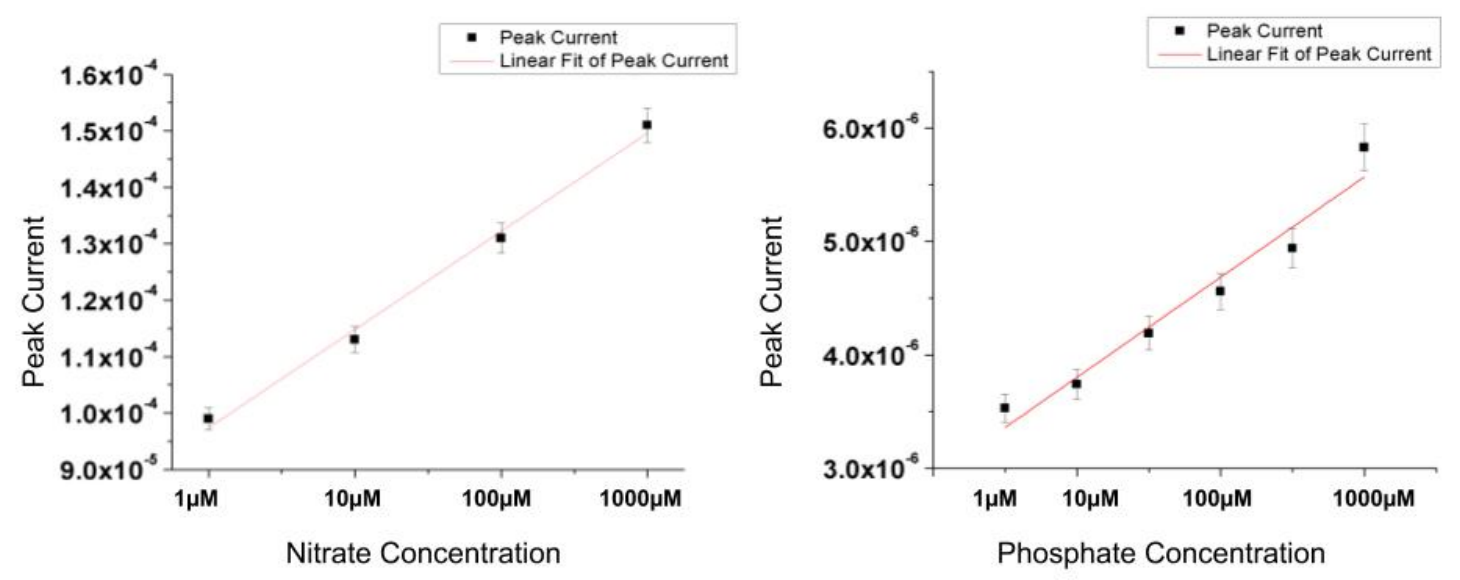

Figure 7.8: Calibration plots for nitrate (left) and phosphate (right) doped PPy sensors with peak currents vs concentration.

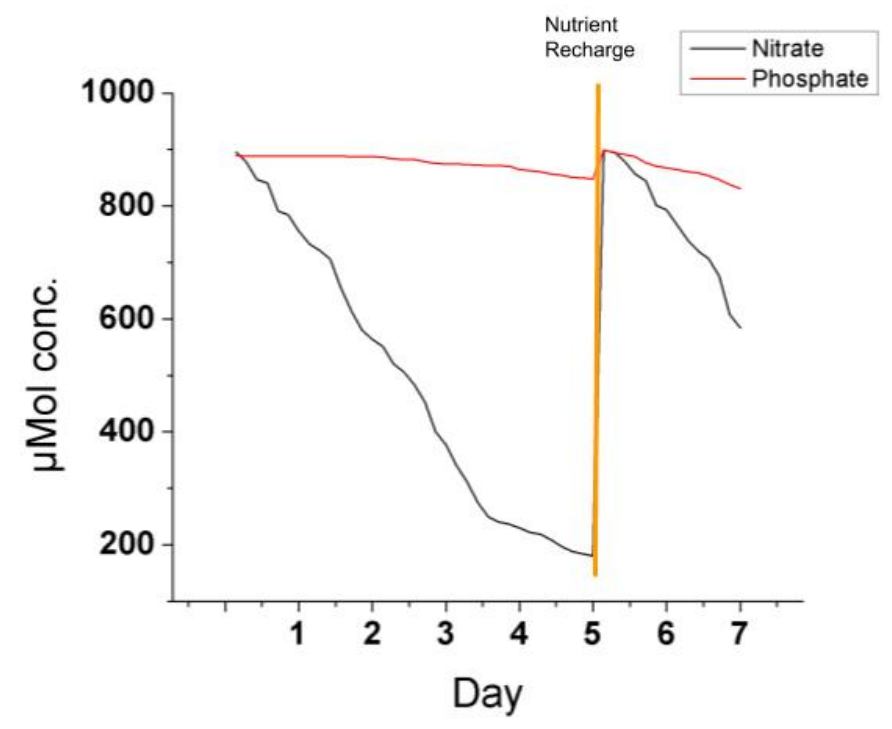

Figure 7.9: Illustration of nutrient uptake by legume plant over a span of approximately 7days with fresh nutrients recharged after day five. Sensors were integrated into microreactor root chambers and immersed in agar nutrient media. 
Table 7.3: Comparison of plant growth parameters when grown using Sense Pod and conventionally. For the experiment Red Crimson Clover seeds were used with each treatment group containing 10 seeds.

\begin{tabular}{|l|l|l|}
\hline Plant Parameters & SensePod & Conventional \\
\hline Germination (Percentage) & 100 & 100 \\
Root Height Avg. (mm) & $23+/-3.72$ & $18+/-4.16$ \\
Shoot Height Avg. (mm) & $48+/-5.68$ & $51+/-4.37$ \\
SPAD Avg. & 23.76 & 12.86 \\
Total soluble sugar (mg/ml) & $0.68+/-0.236$ & $0.7+/-0.080$ \\
Total N & $5.1+/-0.2$ & $5.2+/-0.2$ \\
Chlorophyll A & $0.68+/-0.284$ & $0.65+/-0.29$ \\
Chlorophyll B & $0.28+/-0.1245$ & $0.30+/-0.069$ \\
Total Chlorophyll & $0.96+/-0.406$ & $0.96+/-0.346$ \\
\hline
\end{tabular}

Seed germination and plant growth The seeds were germinated and grown into the germination pod and assessed for germination percentage, average root height, and average SPAD count for each treatment group as shown in table 7.3. The sensors demonstrated the ability to measure real-time nutrient uptake of legume plants in the early stages of their growth (Figure 7.9). Figure 7.10 captures the root morphology over the 7 day observation. As illustrated in figure 7.11, stomata images were captured indicating normal transpiration and plant functional processes. Further, this supports that plants can be grown inside these low-cost devices without any hindrance to growth or performance.

\subsection{SensePod Summary}

In summary, this chapter presented and demonstrated a low-cost simple method to fabricating sensor sheets and multi-ion selective sensors integrated into the sensor germination pod devices. The sheets were achieved by ink-jet printing carbon with nanoparticles and the SensePod device was achieved by layering PDMS sheets between glass slides to obtain the desired microreactor root chamber. The printed sensors measured in 


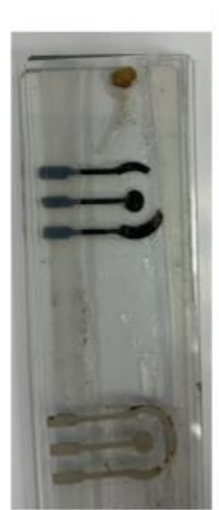

Day 1

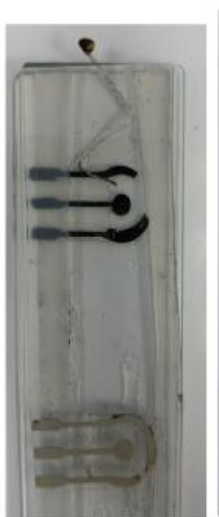

Day 2

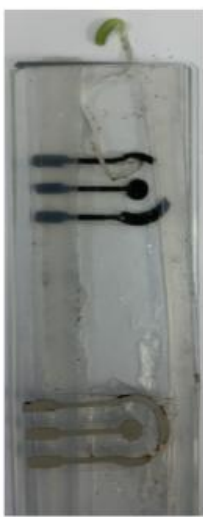

Day 3

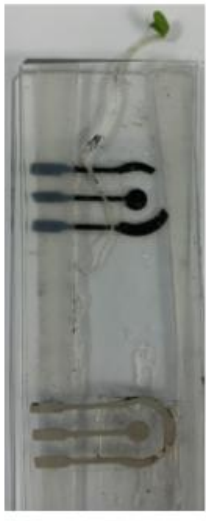

Day 4

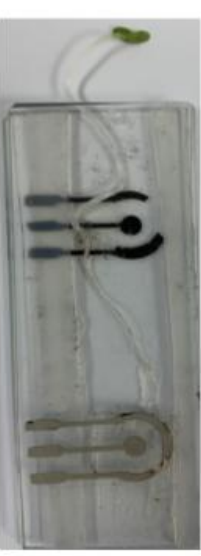

Day 5

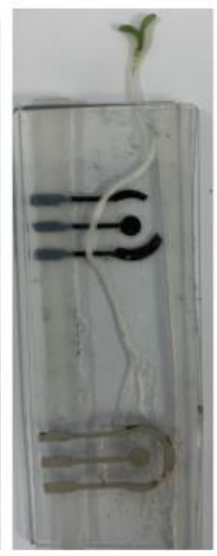

Day 6

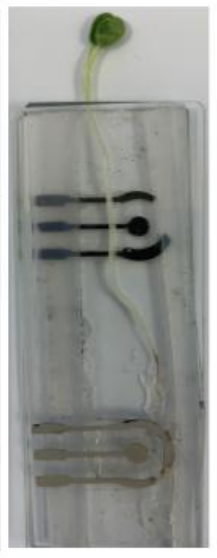

Day 7

Figure 7.10: The use of SensePod to monitor plant root and shoot growth over a period of seven days.

a)

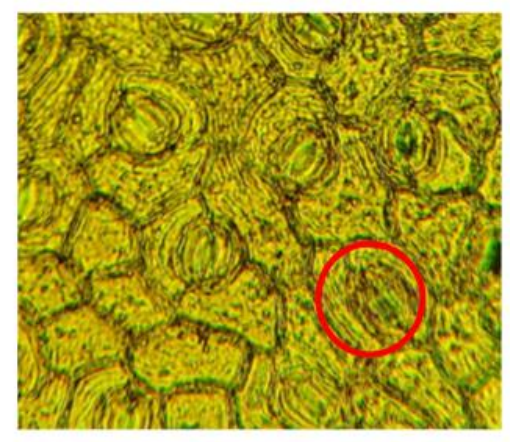

b)

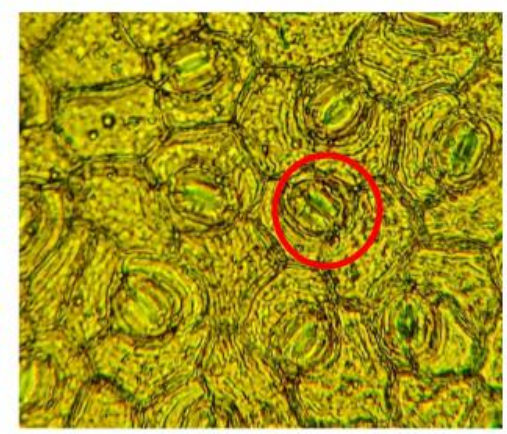

Figure 7.11: a) Microscopic image of leaf epidermis from legume plant grown in the device, revealing normal stoma development and function. The red circles encompass an example stomata. b) microscopic image of leaf epidermis from conventionally grown legume plant. 
situ nitrate and phosphate concentrations inside soil leachate and inside growth medium as legume plant roots grew within the SensePod device. The selective sensors demonstrated high sensitivity and are able to continuously monitor nutrients over a period of approximately 7days. Overall, this chapter demonstrated that low-cost sensor sheets can be fabricated and used for determing soil leachate in real time. It also demonstrated that plants can be grown in uniquely fabricated germination pod devices without any hindrance in normal performance and growth. Such a devices have the potential to aid plant everyday gardeners and scientists for real-time applications in monitoring root-pathogen interaction, drought-resistant plant variety screening, nutrient uptake efficiencies, and rhizosphere microenvironment monitoring. 


\section{CHAPTER 8}

\section{CONCLUSION AND FUTURE WORK}

\subsection{Conclusion}

Climate change, water scarcity, and soil infertility are current challenges and threats to food security. As the global population rises exponentially, food production will need to increase 70 percents in order to satisfy the demand. Since the green revolution, traditional methods for increasing crop yield heavily relied on the excessive input of $\mathrm{N}$ based fertilizers. In the agricultural tech revolution, farmers are seeking to adopt realtime technologies to help optimize production and minimize resources in order to meet this growing demand. This dissertation explores how to increase sensor density in the agricultural framework using low-cost sensors, while also managing major bottlenecks preventing their full commercial adoption for agriculture, accuracy and drift. It also investigated whether low-cost biodegradable printed sensor sheets can result in improved stability, accuracy or drift for use in precision agriculture.

The major objective of this dissertation is to obtain low-cost and high density soil nutrient sensor platforms for precision agriculture.

The envisioned objective will be accomplished through the following goals:

- A. Fabricate and understand the behaviours of disposable sensors when used insitu for real time nutrient analysis

- B. Utilize supervised machine learning for predicting the in-situ sensor response

- C. To use these fabricated disposable sensors in three dimensional plant germination pod systems

The first goal was achieved by fabricating the disposable sensors, then creating an experimental controlled system to evaluate those sensors. After evaluation, the data was 
analysed. The system was also modeled and was compared to gain further insights on the viability of the models. The second goal was achieved by utilizing supervised learning algorithms. The third goal was achieved by incorporating the fabricated sensors into the constructed germination pods and monitoring physical and chemical characteristics of the legume crops that were grown into the pod device.

Since the sensors are in-situ based, the dissertation first sought to provide a basic understanding of soils. Chapter two briefly gives an over view of soils, their compositions, characteristics, and functions in agriculture. The chapter further advocates the importance of understanding soils in order to better manage soil fertility and water usage in agriculture. Due to the excessive utilization of fertilizers and pollution effects on the environment, a growing concern of pollution has warranted the need for technologies that can better monitor soil nutrients and their fate.

Chapter three discusses sensors which can be applied towards precision agriculture to aid in understanding many soil nutrient quality. Given the optical and electrochemical sensors mentioned in chapter three, the chapter suggests the urgent need for incorporating them with IoT, ML and Artificial Intelligence. This would allow real-time monitoring and control of farm systems, thus creating an autonomous network for predicting in-situ nutrient sensor response, there by making it easier for farmers to optimize production and minimize resource utilization. The conventional laboratory methods may offer highly accurate analysis of soil chemistry, in-situ based soil nutrient sensors that offer real-time feedback are needed in order to truly increase the efficiency of farming and managing the environment. As compared to conventional lab instruments for soil nutrient analysis, the chapter identifies that ins-situ based sensors are more advantageous due to their low-cost, and high-density measurement capability for large-area soil nutrient mapping. 
Chapter four investigated implementing printing technologies as an alternative to traditional clean-room technologies for low-cost large volume manufacturing of sensors. The printing techniques discussed in this chapter included roll-to-roll methods and ink-jet printing. Roll-to-roll techniques include screen printing, flexography and gravure printing. The greatest advantage of these methods over traditional clean-room fabrication techniques is the ability to produce large batches of sensors and devices in such a short time using inexpensive materials. Unlike clean-room facilities, which requires vacuum chambers and low particulate spaces for electrode deposition, fabrication of sensors and electrodes via printing method can be accomplished in ambient conditions virtually in any space. Although these sensors are becoming less expensive to manufacture and can provide comparable results to laboratory soil analysis, there still exists a need to understand the effects of soil heterogeneity on the response of both optical and electrochemical sensors. More durable and accurate sensor systems that consider the effects of soils heterogeneity and offer soil specific calibrations are needed to move towards commercializing these platforms.

In chapter five, the printing approach was carried out to fabricate sensors then these sensors were used to understand the effect of soil characteristics on sensor responses. Disposable large scale sensing for soil nutrients in real-time has not been possible because of accuracy and drift. In this chapter, mass soil water content $(\theta \mathrm{m})$, organic matter $(\mathrm{OM})$, particle size (Ps), electrode placement in soil $\left(\mathrm{E}_{\text {place }}\right)$, time after applied moisture (TAM)) and lead length (LL), were selected to investigate their effect on the analytical performance of the printed based soil nitrate sensor system, which was fabricated in house. 


\subsection{Future work}

Future work aims are to address limitations of this dissertation, and to explore printing on alternative substrates such as agricultural textiles. The objective of the work was to achieve high density sensor platforms for precision agriculture based on biodegradable materials. Perhaps future work could explore the use of additional bio-compatible conductive polymers for printed sensors. The sensor performance and soil heterogeneity experiments were limited to the sandy soil textural class, two particle sizes and four different contents of organic matter. These limitations may inspire future studies of other particle sizes and textural classes of soil such as silt, loam and clay, and further include the role of microbiota on the degradation of in-situ electrodes. This could improve the overall stability of the electrodes and possibly increase the number of cycles the sensors could reproduce. Exploring additional substrates such as weed block fabrics would also prove beneficial. Weed block fabric acts as a weed suppressor between crops and remains on soil for long periods of time, making it an ideal substrate for a high den-

sity sensor platform. Further studies on printing on fabrics, electrode adhesion quality to fabrics, and overall mechanical integrity would benefit future studies attempting to translate the printed structures onto these textiles. 


\section{BIBLIOGRAPHY}

[1] N. C. Brady and R. R. Weil, The Nature and Properties of Soils. Upper Saddle River, NJ: Pearson Education Inc., 14. ed., internat. ed. ed., 2008.

[2] N. Schröder, Three-dimensional solute transport modeling in coupled soil and plant root systems. Forschungszentrum Jülich, 2014.

[3] W. C. Willett, J. Rockström, B. Loken, M. Springmann, T. N. Lang, Sonja, Vermeulen, and J. A. Rivera, "Our food in the anthropocene : The eat-lancet commission on healthy diets from sustainable food systems," 2019.

[4] USDA, "World agricultural production," Circular Series, 2019.

[5] H. C. J. Godfray, J. R. Beddington, I. R. Crute, L. Haddad, D. Lawrence, J. F. Muir, J. Pretty, S. Robinson, S. M. Thomas, and C. Toulmin, "Food security: the challenge of feeding 9 billion people," science, vol. 327, no. 5967, pp. 812-818, 2010.

[6] W. Zhang, "Global pesticide use: Profile, trend, cost/benefit and more," Proceedings of the International Academy of Ecology and Environmental Sciences, vol. 8, no. 1, p. 1, 2018.

[7] H.-J. Kim, K. A. Sudduth, and J. W. Hummel, "Soil macronutrient sensing for precision agriculture," Journal of Environmental Monitoring, vol. 11, no. 10, pp. 1810-1824, 2009.

[8] K. Sudduth, J. Hummel, and S. Birrell, "Sensors for site-specific management," The state of site-specific management for agriculture, no. thestateofsites, pp. 183210, 1997.

[9] V. Adamchuk, E. Lund, B. Sethuramasamyraja, M. Morgan, A. Dobermann, and D. Marx, "Direct measurement of soil chemical properties on-the-go using ionselective electrodes," Computers and Electronics in Agriculture, vol. 48, no. 3, pp. 272-294, 2005.

[10] S. Laskar and S. Mukherjee, "Optical sensing methods for assessment of soil macronutrients and other properties for application in precision agriculture: a review," ADBU journal of Engineering Technology, vol. 4, 2016.

[11] D. P. Schachtman and R. Shin, "Nutrient sensing and signaling: Npks," Annu. Rev. Plant Biol., vol. 58, pp. 47-69, 2007. 
[12] M. H. Ward, R. R. Jones, J. D. Brender, T. M. De Kok, P. J. Weyer, B. T. Nolan, C. M. Villanueva, and S. G. Van Breda, "Drinking water nitrate and human health: an updated review," International journal of environmental research and public health, vol. 15, no. 7, p. 1557, 2018.

[13] A. Ball, W. S. Wilson, W. Wilson, and R. Hinton, Managing risks of nitrates to humans and the environment. No. 237, Woodhead Publishing, 1999.

[14] C. W. Act, "Clean water act," EPA's Office, 2008.

[15] R. A. V. Rossel and J. Bouma, "Soil sensing: A new paradigm for agriculture," Agricultural Systems, vol. 148, pp. 71-74, 2016.

[16] N. Chandel, C. Mehta, V. Tewari, and B. Nare, "Digital map-based site-specific granular fertilizer application system," CURRENT SCIENCE, vol. 111, no. 7, p. 1208, 2016.

[17] J. V. Sinfield, D. Fagerman, and O. Colic, "Evaluation of sensing technologies for on-the-go detection of macro-nutrients in cultivated soils," Computers and Electronics in Agriculture, vol. 70, no. 1, pp. 1-18, 2010.

[18] C. Dixon, A. H. Ng, R. Fobel, M. B. Miltenburg, and A. R. Wheeler, "An inkjet printed, roll-coated digital microfluidic device for inexpensive, miniaturized diagnostic assays," Lab on a Chip, vol. 16, no. 23, pp. 4560-4568, 2016.

[19] J. Ozhikandathil, S. Badilescu, and M. Packirisamy, "Polymer composite optically integrated lab on chip for the detection of ammonia," Journal of The Electrochemical Society, vol. 165, no. 8, pp. B3078-B3083, 2018.

[20] M. Nocita, A. Stevens, B. van Wesemael, M. Aitkenhead, M. Bachmann, B. Barthès, E. B. Dor, D. J. Brown, M. Clairotte, A. Csorba, et al., "Soil spectroscopy: An alternative to wet chemistry for soil monitoring," in Advances in agronomy, vol. 132, pp. 139-159, Elsevier, 2015.

[21] J. M. Soriano-Disla, L. J. Janik, R. A. Viscarra Rossel, L. M. Macdonald, and M. J. McLaughlin, "The performance of visible, near-, and mid-infrared reflectance spectroscopy for prediction of soil physical, chemical, and biological properties," Applied Spectroscopy Reviews, vol. 49, no. 2, pp. 139-186, 2014.

[22] S. Shibusawa, H. Sato, A. Sasao, S. Hirako, and A. Otomo, "A revised soil spectrophotometer," IFAC Proceedings Volumes, vol. 33, no. 29, pp. 231-236, 2000. 
[23] S. J. Birrell and J. W. Hummel, "Real-time multi isfet/fia soil analysis system with automatic sample extraction," Computers and Electronics in Agriculture, vol. 32, no. 1, pp. 45-67, 2001.

[24] X. An, M. Li, L. Zheng, Y. Liu, and H. Sun, "A portable soil nitrogen detector based on nirs," Precision agriculture, vol. 15, no. 1, pp. 3-16, 2014.

[25] J. B. Reeves III, "Near-versus mid-infrared diffuse reflectance spectroscopy for soil analysis emphasizing carbon and laboratory versus on-site analysis: where are we and what needs to be done?," Geoderma, vol. 158, no. 1-2, pp. 3-14, 2010.

[26] Z. Shi, W. Ji, R. Viscarra Rossel, S. Chen, and Y. Zhou, "Prediction of soil organic matter using a spatially constrained local partial least squares regression and the c hinese vis-nir spectral library," European Journal of Soil Science, vol. 66, no. 4, pp. 679-687, 2015.

[27] M. Ehsani, S. Upadhyaya, D. Slaughter, S. Shafii, and M. Pelletier, "A nir technique for rapid determination of soil mineral nitrogen," Precision agriculture, vol. 1, no. 2, pp. 219-236, 1999.

[28] M. Ehsani, S. Upadhyaya, D. Slaughter, L. Protsailo, W. Fawcett, et al., "Quantitative measurement of soil nitrate content using mid-infrared diffuse reflectance spectroscopy.," Quantitative measurement of soil nitrate content using midinfrared diffuse reflectance spectroscopy., pp. 1-15, 2000.

[29] R. V. Rossel, D. Walvoort, A. McBratney, L. J. Janik, and J. Skjemstad, "Visible, near infrared, mid infrared or combined diffuse reflectance spectroscopy for simultaneous assessment of various soil properties," Geoderma, vol. 131, no. 1-2, pp. 59-75, 2006.

[30] S. Shibusawa, "On-line real time soil sensor," in Proceedings 2003 IEEE/ASME International Conference on Advanced Intelligent Mechatronics (AIM 2003), vol. 2, pp. 1061-1066, IEEE, 2003.

[31] C. D. Christy, P. Drummond, and D. A. Laird, "An on-the-go spectral reflectance sensor for soil," in 2003 ASAE Annual Meeting, p. 1, American Society of Agricultural and Biological Engineers, 2003.

[32] B. Jahn, R. Linker, S. Upadhyaya, A. Shaviv, D. Slaughter, and I. Shmulevich, "Mid-infrared spectroscopic determination of soil nitrate content," Biosystems Engineering, vol. 94, no. 4, pp. 505-515, 2006. 
[33] M. F. Baumgardner, L. F. Silva, L. L. Biehl, and E. R. Stoner, "Reflectance properties of soils," in Advances in agronomy, vol. 38, pp. 1-44, Elsevier, 1986.

[34] K. M. Smith and P. Holroyd, Engineering Principles for Electrical Technicians: The Commonwealth and International Library: Electrical Engineering Division. Elsevier, 2013.

[35] N. Yefimov and S. Naboychenko, Handbook of non-ferrous metal powders: technologies and applications. Elsevier, 2009.

[36] P. K. Sekhar and J. S. Kysar, "An electrochemical ammonia sensor on paper substrate," Journal of The Electrochemical Society, vol. 164, no. 4, pp. B113-B117, 2017.

[37] L. Burton, N. Dave, R. Fernandez, K. Jayachandran, and S. Bhansali, "Smart gardening iot soil sheets for real-time nutrient analysis," Journal of The Electrochemical Society, vol. 165, no. 8, pp. B3157-B3162, 2018.

[38] Y. Mekonnen, L. Burton, A. Sarwat, and S. Bhansali, "Iot sensor network approach for smart farming: An application in food, energy and water system," in 2018 IEEE Global Humanitarian Technology Conference (GHTC), pp. 1-5, IEEE, 2018.

[39] A. A. Aniley, N. Kumar, A. Kumar, R. E. Fernandez, and S. Bhansali, "Thin film dual probe heat pulse (DPHP) micro heater network for soil moisture content estimation in smart agriculture," Journal of The Electrochemical Society, vol. 166, no. 2, pp. B63-B67, 2019.

[40] K. Sibley, T. Astatkie, and G. Brewster, "field scale validation of an automated soil nitrate extraction and measurement system," Precision agriculture, 2009.

[41] M. Smolka, D. Puchberger-Enengl, M. Bipoun, A. Klasa, M. Kiczkajlo, W. Śmiechowski, P. Sowiński, C. Krutzler, F. Keplinger, and M. Vellekoop, “A mobile lab-on-a-chip device for on-site soil nutrient analysis," Precision agriculture, vol. 18, no. 2, pp. 152-168, 2017.

[42] K. N. Mikhelson, Ion-selective electrodes, vol. 81. Springer, 2013.

[43] Y. Chen, X.-L. Guo, J.-C. Yan, Y.-F. Zhao, Y. Pang, J.-M. Jian, M. Morikado, X.M. Wu, Y. Yang, and T.-L. Ren, "Toward an in situ phosphate sensor in natural 
waters using a microfluidic flow loop analyzer," Journal of The Electrochemical Society, vol. 165, no. 14, pp. B737-B745, 2018.

[44] Y. Li, T. Jiang, X. Yu, and H. Yang, "Phosphate sensor using molybdenum," Journal of The Electrochemical Society, vol. 163, no. 9, pp. B479-B484, 2016.

[45] T. H. V. Kumar and A. K. Sundramoorthy, "Non-enzymatic electrochemical detection of urea on silver nanoparticles anchored nitrogen-doped single-walled carbon nanotube modified electrode," Journal of The Electrochemical Society, vol. 165, no. 8, pp. B3006-B3016, 2018.

[46] J. K. F. van Staden, R.-G. Nuta, and G.-L. T. (Arnold), "Determination of nitrite from water catchment areas using graphite based electrodes," Journal of The Electrochemical Society, vol. 165, no. 13, pp. B565-B570, 2018.

[47] K. Tyszczuk-Rotko and K. Jedruchniewicz, "Ultrasensitive sensor for uranium monitoring in water ecosystems," Journal of The Electrochemical Society, vol. 166, no. 10, pp. B837-B844, 2019.

[48] C. Lobsey, R. V. Rossel, A. McBratney, et al., "An automated system for rapid infield soil nutrient testing," in 19th World Congress of Soil Science, Soil Solutions for a Changing World, pp. 1-6, 2010.

[49] C. Esmaeili, P. Norouzi, M. S. Zar, M. Eskandari, F. Faridbod, and M. R. Ganjali, "A FFT square wave voltammetry sensing method for highly sensitive detection of phytic acid using a cerium oxide nanoparticles decorated graphene oxide," Journal of The Electrochemical Society, vol. 166, no. 15, pp. B1630-B1636, 2019.

[50] R. Stirzaker, N. Car, and E. Christen, "A traffic light soil water sensor for resource poor farmers: proof of concept," Final project report. Australian Centre for International Agricultural Research (ACIAR), Canberra. URL: http://aciar. gov. au/files/aciar_traffic_light_final_report_sept_14_2_2. pdf (Accessed 9 May 2017), 2014.

[51] C. R. Lobsey, R. V. Rossel, and A. B. Mcbratney, "Proximal soil nutrient sensing using electrochemical sensors," in Proximal soil sensing, pp. 77-88, Springer, 2010 .

[52] H. J. Kim, Ion-selective electrodes for simultaneous real-time analysis for soil macronutrients. PhD thesis, University of Missouri-Columbia, 2006. 
[53] S. Aravamudhan and S. Bhansali, "Development of micro-fluidic nitrate-selective sensor based on doped-polypyrrole nanowires," Sensors and Actuators B: Chemical, vol. 132, no. 2, pp. 623-630, 2008.

[54] K. J. Sibley, G. R. Brewster, T. Astatkie, J. F. Adsett, and P. C. Struik, "In-field measurement of soil nitrate using an ion-selective electrode," in Advances in measurement systems, IntechOpen, 2010.

[55] B. Sun and P. G. Fitch, "Nitrate ion-selective sensor based on electrochemically prepared conducting polypyrrole films," Electroanalysis, vol. 9, no. 6, pp. 494497, 1997.

[56] A. Antonacci, F. Arduini, D. Moscone, G. Palleschi, and V. Scognamiglio, "Nanostructured (bio) sensors for smart agriculture," TrAC Trends in Analytical Chemistry, vol. 98, pp. 95-103, 2018.

[57] F. Hofmeister, "On the theory of the effects of salts arch," Exp. Pathol. Pharmakol, vol. 24, pp. 247-260, 1888.

[58] N. M. Nasrabadi, "Pattern recognition and machine learning," Journal of Electronic Imaging, vol. 16, 2007.

[59] Y. Mekonnen, S. Namuduri, L. Burton, A. Sarwat, and S. Bhansali, "Machine learning techniques in wireless sensor network based precision agriculture," Journal of The Electrochemical Society, vol. 167, no. 3, p. 037522, 2020.

[60] S. Marsland, Machine Learning: An Algorithmic Perspective. Chapman \& Hall/CRC, 1st ed., 2009.

[61] G. James, R. Tibshirani, D. Witten, and T. Hastie, An introduction to statistical learning: with applications in R, vol. 103 of Springer texts in statistics. 2013.

[62] A. Kataria and M. D. Singh, "A review of data classification using k-nearest neighbour algorithm," June 2013.

[63] Plastesmart, "Printed electronics sector takes a hard look at the flexible future."

[64] IDTechEx, "Printed and flexible sensors market - global industry size, share, trends, analysis, and forecasts." 
[65] R. Gupta, S. Walia, M. Hösel, J. Jensen, D. Angmo, F. C. Krebs, and G. U. Kulkarni, "Solution processed large area fabrication of ag patterns as electrodes for flexible heaters, electrochromics and organic solar cells," Journal of Materials Chemistry A, vol. 2, no. 28, pp. 10930-10937, 2014.

[66] M. Layani, P. Darmawan, W. L. Foo, L. Liu, A. Kamyshny, D. Mandler, S. Magdassi, and P. S. Lee, "Nanostructured electrochromic films by inkjet printing on large area and flexible transparent silver electrodes," Nanoscale, vol. 6, no. 9, pp. 4572-4576, 2014.

[67] U. Männl, C. Van Den Berg, B. Magunje, M. Härting, D. Britton, S. Jones, M. Van Staden, and M. Scriba, "Nanoparticle composites for printed electronics," Nanotechnology, vol. 25, no. 9, p. 094004, 2014.

[68] K. J. Lee, B. H. Jun, T. H. Kim, and J. Joung, "Direct synthesis and inkjetting of silver nanocrystals toward printed electronics," Nanotechnology, vol. 17, no. 9, p. 2424, 2006.

[69] IDTechEx, "Printed and flexible sensors 2014-2024: Technologies, players, forecasts.."

[70] B. Roth, R. Søndergaard, and F. Krebs, "Roll-to-roll printing and coating techniques for manufacturing large-area flexible organic," in Handbook of Flexible Organic Electronics: Materials, Manufacturing and Applications, pp. 171-192, Woodhead Publishing, 2014.

[71] T. Dunn, "3 - flexographic printing," in Flexible Packaging (T. Dunn, ed.), pp. 27 - 37, Oxford: William Andrew Publishing, 2015.

[72] A. Hübler, B. Trnovec, T. Zillger, M. Ali, N. Wetzold, M. Mingebach, A. Wagenpfahl, C. Deibel, and V. Dyakonov, "Printed paper photovoltaic cells," Advanced Energy Materials, vol. 1, no. 6, pp. 1018-1022, 2011.

[73] F. C. Krebs, J. Fyenbo, and M. Jørgensen, "Product integration of compact rollto-roll processed polymer solar cell modules: methods and manufacture using flexographic printing, slot-die coating and rotary screen printing," Journal of Materials Chemistry, vol. 20, no. 41, pp. 8994-9001, 2010.

[74] M. Hösel, R. R. Søndergaard, M. Jørgensen, and F. C. Krebs, "Comparison of uv-curing, hotmelt, and pressure sensitive adhesive as roll-to-r oll encapsulation methods for polymer solar cells," Advanced Engineering Materials, vol. 15, no. 11, pp. 1068-1075, 2013. 
[75] J.-S. Yu, I. Kim, J.-S. Kim, J. Jo, T. T. Larsen-Olsen, R. R. Søndergaard, M. Hösel, D. Angmo, M. Jørgensen, and F. C. Krebs, "Silver front electrode grids for ito-free all printed polymer solar cells with embedded and raised topographies, prepared by thermal imprint, flexographic and inkjet roll-to-roll processes," Nanoscale, vol. 4, no. 19, pp. 6032-6040, 2012.

[76] M. Hambsch, K. Reuter, M. Stanel, G. Schmidt, H. Kempa, U. Fügmann, U. Hahn, and A. Hübler, "Uniformity of fully gravure printed organic field-effect transistors," Materials Science and Engineering: B, vol. 170, no. 1-3, pp. 93-98, 2010.

[77] P. Kopola, M. Tuomikoski, R. Suhonen, and A. Maaninen, "Gravure printed organic light emitting diodes for lighting applications," Thin Solid Films, vol. 517, no. 19 , pp. 5757-5762, 2009.

[78] J.-M. Verilhac, M. Benwadih, A.-L. Seiler, S. Jacob, C. Bory, J. Bablet, M. Heitzman, J. Tallal, L. Barbut, P. Frère, et al., "Step toward robust and reliable amorphous polymer field-effect transistors and logic functions made by the use of roll to roll compatible printing processes," Organic Electronics, vol. 11, no. 3, pp. 456-462, 2010.

[79] C. M. Amb, M. R. Craig, U. Koldemir, J. Subbiah, K. R. Choudhury, S. A. Gevorgyan, M. Jørgensen, F. C. Krebs, F. So, and J. R. Reynolds, "Aesthetically pleasing conjugated polymer: fullerene blends for blue-green solar cells via roll-to-roll processing," ACS applied materials \& interfaces, vol. 4, no. 3, pp. 1847-1853, 2012.

[80] D. Angmo, M. Hösel, and F. C. Krebs, "All solution processing of ito-free organic solar cell modules directly on barrier foil," Solar energy materials and solar cells, vol. 107, pp. 329-336, 2012.

[81] E. Bundgaard, O. Hagemann, M. Manceau, M. Jørgensen, and F. C. Krebs, "Low band gap polymers for roll-to-roll coated polymer solar cells," Macromolecules, vol. 43, no. 19, pp. 8115-8120, 2010.

[82] H. F. Dam and F. C. Krebs, "Simple roll coater with variable coating and temperature control for printed polymer solar cells," Solar Energy Materials and Solar Cells, vol. 97, pp. 191-196, 2012.

[83] N. Espinosa, F. O. Lenzmann, S. Ryley, D. Angmo, M. Hösel, R. R. Søndergaard, D. Huss, S. Dafinger, S. Gritsch, J. M. Kroon, et al., "Opv for mobile applications: an evaluation of roll-to-roll processed indium and silver free polymer solar 
cells through analysis of life cycle, cost and layer quality using inline optical and functional inspection tools," Journal of Materials Chemistry A, vol. 1, no. 24, pp. 7037-7049, 2013.

[84] Y. Galagan, J.-E. J. Rubingh, R. Andriessen, C.-C. Fan, P. W. Blom, S. C. Veenstra, and J. M. Kroon, "Ito-free flexible organic solar cells with printed current collecting grids," Solar Energy Materials and Solar Cells, vol. 95, no. 5, pp. 1339-1343, 2011.

[85] G. S. Ryu, J. S. Kim, S. H. Jeong, and C. K. Song, "A printed otft-backplane for amoled display," Organic Electronics, vol. 14, no. 4, pp. 1218-1224, 2013.

[86] A. Lange, W. Schindler, M. Wegener, K. Fostiropoulos, and S. Janietz, "Inkjet printed solar cell active layers prepared from chlorine-free solvent systems," Solar energy materials and solar cells, vol. 109, pp. 104-110, 2013.

[87] S. Chung, S. O. Kim, S.-K. Kwon, C. Lee, and Y. Hong, "All-inkjet-printed organic thin-film transistor inverter on flexible plastic substrate," IEEE electron device letters, vol. 32, no. 8, pp. 1134-1136, 2011.

[88] D. Tobjörk, N. Kaihovirta, T. Mäkelä, F. Pettersson, and R. Österbacka, "Allprinted low-voltage organic transistors," Organic Electronics, vol. 9, no. 6, pp. 931-935, 2008.

[89] H. Gorter, M. Coenen, M. Slaats, M. Ren, W. Lu, C. Kuijpers, and W. Groen, "Toward inkjet printing of small molecule organic light emitting diodes," Thin Solid Films, vol. 532, pp. 11-15, 2013.

[90] S. G. R. Avuthu, "Implementation of traditional printing techniques for the development of flexible printed sensors," 2015.

[91] J.-T. Kwon, S.-H. Eom, B.-S. Moon, J.-K. Shin, K.-S. Kim, S.-H. Lee, and Y.-S. Lee, "Studies on printing inks containing poly [2-methoxy-5-(2-ethylhexyl-oxyl)1, 4-phenylenevinylene] as an emissive material for the fabrication of polymer light-emitting diodes by inkjet printing," Bulletin of the Korean Chemical Society, vol. 33, no. 2, pp. 464-468, 2012.

[92] J. W. Erisman, J. N. Galloway, S. Seitzinger, A. Bleeker, N. B. Dise, A. R. Petrescu, A. M. Leach, and W. de Vries, "Consequences of human modification of the global nitrogen cycle," Philosophical Transactions of the Royal Society B: Biological Sciences, vol. 368, no. 1621, p. 20130116, 2013. 
[93] USEPA, "Consumer factsheet on: Nitrates/nitrites," 2003.

[94] NSF, "Dear colleague letter: Signals in the soil."

[95] T. Miller, P.-h. Le Besnerais, and H. Malaurie, "Soil chemistry sensor," Aug. 15 2017. US Patent 9,733,206.

[96] M. d. 1. A. A. Pérez, L. P. Marın, J. C. Quintana, and M. Yazdani-Pedram, "Influence of different plasticizers on the response of chemical sensors based on polymeric membranes for nitrate ion determination," Sensors and Actuators B: Chemical, vol. 89, no. 3, pp. 262-268, 2003.

[97] B. Schazmann and D. Diamond, "Improved nitrate sensing using ion selective electrodes based on urea-calixarene ionophores," New Journal of Chemistry, vol. 31, no. 4, pp. 587-592, 2007.

[98] A. S. Watts, V. G. Gavalas, A. Cammers, P. S. Andrada, M. Alajarín, and L. G. Bachas, "Nitrate-selective electrode based on a cyclic bis-thiourea ionophore," Sensors and Actuators B: Chemical, vol. 121, no. 1, pp. 200-207, 2007.

[99] J. Bobacka, "Potential stability of all-solid-state ion-selective electrodes using conducting polymers as ion-to-electron transducers," Analytical chemistry, vol. 71, no. 21, pp. 4932-4937, 1999.

[100] J. Bobacka, "Conducting polymer-based solid-state ion-selective electrodes," Electroanalysis: An International Journal Devoted to Fundamental and Practical Aspects of Electroanalysis, vol. 18, no. 1, pp. 7-18, 2006.

[101] E. M. Bomar, G. S. Owens, and G. M. Murray, "Nitrate ion selective electrode based on ion imprinted poly (n-methylpyrrole)," Chemosensors, vol. 5, no. 1, p. 2, 2017.

[102] C. K. Sung and L. Keun-Sun, "Polypyrrole modified electrode as a nitrate sensor," Bulletin of the Korean Chemical Society, vol. 11, no. 2, pp. 124-126, 1990.

[103] R. S. Hutchins and L. G. Bachas, "Nitrate-selective electrode developed by electrochemically mediated imprinting/doping of polypyrrole," Analytical Chemistry, vol. 67, no. 10, pp. 1654-1660, 1995. 
[104] L. Burton, K. Jayachandran, and S. Bhansali, "The "real-time" revolution for in situ soil nutrient sensing: A review,' Journal of The Electrochemical Society, vol. 167, no. 3, p. 037569, 2020.

[105] X.-Z. Song, C.-X. Zhao, X.-L. Wang, and J. Li, "Study of nitrate leaching and nitrogen fate under intensive vegetable production pattern in northern china," Comptes Rendus Biologies, vol. 332, no. 4, pp. 385 - 392, 2009.

[106] P. M. Glibert, M. Zhou, M. Zhu, and M. A. Burford, "Preface to the special issue on eutrophication and habs: the geohab approach," Chinese Journal of Oceanology and Limnology, vol. 29, no. 4, p. 719, 2011.

[107] T. P. R, “Credibility in soil testing analytical results," 1998.

[108] L. Zhang, M. Zhang, H. Ren, P. Pu, P. Kong, and H. Zhao, "Comparative investigation on soil nitrate-nitrogen and available potassium measurement capability by using solid-state and pvc ise," Computers and Electronics in Agriculture, vol. 112, pp. 83-91, 2015.

[109] J. V. Sinfield, D. Fagerman, and O. Colic, "Evaluation of sensing technologies for on-the-go detection of macro-nutrients in cultivated soils," Computers and Electronics in Agriculture, vol. 70, no. 1, pp. 1 - 18, 2010.

[110] J. Artigas, A. Beltran, C. Jiménez, A. Baldi, R. Mas, C. Domınguez, and J. Alonso, "Application of ion sensitive field effect transistor based sensors to soil analysis," Computers and Electronics in Agriculture, vol. 31, no. 3, pp. 281 - 293, 2001.

[111] E. D. Wood, F. A. J. Armstrong, and F. A. Richards, "Determination of nitrate in sea water by cadmium-copper reduction to nitrite," Journal of the Marine Biological Association of the United Kingdom, vol. 47, no. 1, p. 23-31, 1967.

[112] L. C. Green, D. A. Wagner, J. Glogowski, P. L. Skipper, J. S. Wishnok, and S. R. Tannenbaum, "Analysis of nitrate, nitrite, and [15n]nitrate in biological fluids," Analytical Biochemistry, vol. 126, no. 1, pp. 131 - 138, 1982.

[113] N. K. Cortas and N. W. Wakid, "Determination of inorganic nitrate in serum and urine by a kinetic cadmium-reduction method," Clinical chemistry, vol. 36, no. 8, pp. 1440-1443, 1990.

[114] S. A. Everett, M. F. Dennis, G. M. Tozer, V. E. Prise, P. Wardman, and M. R. Stratford, "Nitric oxide in biological fluids: analysis of nitrite and nitrate by high- 
performance ion chromatography," Journal of Chromatography A, vol. 706, no. 1, pp. 437 - 442, 1995. International Ion Chromatographic Symposium 1994.

[115] J. Mulik, R. Puckett, D. Williams, and E. Sawicki, "Ion chromatographic analysis of sulfate and nitrate in ambient aerosols," Analytical Letters, vol. 9, no. 7, pp. 653-663, 1976.

[116] M. I. Helaleh and T. Korenaga, "Ion chromatographic method for simultaneous determination of nitrate and nitrite in human saliva," Journal of Chromatography B: Biomedical Sciences and Applications, vol. 744, no. 2, pp. 433-437, 2000.

[117] R.-J. Yang, C.-C. Liu, Y.-N. Wang, H.-H. Hou, and L.-M. Fu, “A comprehensive review of micro-distillation methods," Chemical Engineering Journal, vol. 313, pp. 1509-1520, 2017.

[118] P. A. Perez, H. Hintelman, W. Quiroz, and M. A. Bravo, "Critical evaluation of distillation procedure for the determination of methylmercury in soil samples," Chemosphere, vol. 186, pp. 570 - 575, 2017.

[119] M. Zhang, R. E. Karamanos, L. M. Kryzanowski, K. R. Cannon, and T. W. Goddard, "A single measurement to predict potential mineralizable nitrogen," Communications in Soil Science and Plant Analysis, vol. 33, no. 15-18, pp. 35173530, 2002.

[120] C. Campbell, Y. Jamel, A. Jalil, and J. Schoenau, "Use of hot kcl-nh4-n to estimate fertilizer n requirements," Canadian Journal of Soil Science, vol. 77, no. 2, pp. 161-166, 1997.

[121] S. DELIN, B. STENBERG, A. NYBERG, and L. BROHEDE, "Potential methods for estimating nitrogen fertilizer value of organic residues," Soil Use and Management, vol. 28, no. 3, pp. 283-291.

[122] D. A. Fagerman, Exploration of Time-Resolved Raman analysis for on-the-go nitrate sensing. PhD thesis, Purdue University, 2010.

[123] A. T. Winstead, S. H. Norwood, T. W. Griffin, M. Runge, A. M. Adrian, J. Fulton, and J. Kelton, "Adoption and use of precision agriculture technologies by practitioners," in Proc. the 10th International Conference on Precision Agriculture, pp. 18-21, Citeseer, 2010. 
[124] J. Ye, B. Chen, Q. Liu, and Y. Fang, "A precision agriculture management system based on internet of things and webgis," in 201321 st International Conference on Geoinformatics, pp. 1-5, IEEE, 2013.

[125] W. Zhang, "Study about iot's application in" digital agriculture" construction," in 2011 International Conference on Electrical and Control Engineering, pp. 25782581, IEEE, 2011.

[126] I. Mohanraj, K. Ashokumar, and J. Naren, "Field monitoring and automation using iot in agriculture domain," Procedia Computer Science, vol. 93, pp. 931-939, 2016.

[127] J. Ma, X. Zhou, S. Li, and Z. Li, "Connecting agriculture to the internet of things through sensor networks," in 2011 international conference on internet of things and 4th international conference on cyber, physical and social computing, pp. 184-187, IEEE, 2011.

[128] Y. Bo and H. Wang, "The application of cloud computing and the internet of things in agriculture and forestry," in 2011 International Joint Conference on Service Sciences, pp. 168-172, IEEE, 2011.

[129] P. P. Jayaraman, D. Palmer, A. Zaslavsky, and D. Georgakopoulos, "Do-ityourself digital agriculture applications with semantically enhanced iot platform," in 2015 IEEE tenth international conference on intelligent sensors, sensor networks and information processing (ISSNIP), pp. 1-6, IEEE, 2015.

[130] D. Yan-e, "Design of intelligent agriculture management information system based on iot," in 2011 Fourth International Conference on Intelligent Computation Technology and Automation, vol. 1, pp. 1045-1049, IEEE, 2011.

[131] G. Pandey, R. Kumar, and R. J. Weber, "Real time detection of soil moisture and nitrates using on-board in-situ impedance spectroscopy," in 2013 IEEE International Conference on Systems, Man, and Cybernetics, pp. 1081-1086, IEEE, 2013.

[132] J. L. Riquelme, F. Soto, J. Suardíaz, P. Sánchez, A. Iborra, and J. Vera, "Wireless sensor networks for precision horticulture in southern spain," Computers and electronics in agriculture, vol. 68, no. 1, pp. 25-35, 2009.

[133] M. E. E. Alahi, A. Nag, S. C. Mukhopadhyay, and L. Burkitt, "A temperaturecompensated graphene sensor for nitrate monitoring in real-time application," Sensors and Actuators A: Physical, vol. 269, pp. 79-90, 2018. 
[134] E. Martini, U. Werban, S. Zacharias, M. Pohle, P. Dietrich, and U. Wollschläger, "Repeated electromagnetic induction measurements for mapping soil moisture at the field scale: Validation with data from a wireless soil moisture monitoring network," Hydrology and Earth System Sciences, vol. 21, no. 1, p. 495, 2017.

[135] J. L. Riquelme, F. Soto, J. Suardíaz, P. Sánchez, A. Iborra, and J. Vera, "Wireless sensor networks for precision horticulture in southern spain," Computers and Electronics in Agriculture, vol. 68, no. 1, pp. 25 - 35, 2009.

[136] O. Green, E. S. Nadimi, V. Blanes-Vidal, R. N. Jørgensen, I. M. D. Storm, and C. G. Sørensen, "Monitoring and modeling temperature variations inside silage stacks using novel wireless sensor networks," Computers and Electronics in Agriculture, vol. 69, no. 2, pp. 149 - 157, 2009.

[137] L. Li, S. Yang, L. Wang, and X. Gao, "The greenhouse environment monitoring system based on wireless sensor network technology," in 2011 IEEE International Conference on Cyber Technology in Automation, Control, and Intelligent Systems, pp. 265-268, March 2011.

[138] Z. R. G. G. F. Youbing and L. Chengfei., "Realization of communication in wireless monitoring system in greenhouse based on ieee802.15.4," Transactions of the Chinese Society for Agricultural Machinery, 2008.

[139] e. a. Yihua, Cai, "Design and test of nodes for farmland data acquisition based on wireless sensor network," Transactions of the Chinese Society of Agricultural Engineering, 2009.

[140] R. Z. Feng Youbing and G. Gu., "Application of wireless sensor network in watersaving irrigation," China Rural Water and Hydropower, 2007.

[141] B. H. Srinivasa Rao, P.C., "Energy efficient clustering algorithms for wireless sensor networks: novel chemical reaction optimization approach," Wireless Networks.

[142] M. e. a. Nishita, "Ror2 signaling regulates golgi structure and transport through ift20 for tumor invasiveness," Scientific Reports.

[143] M. D. R. K. Klaus Butterbach-Bahl, Elizabeth M. Baggs and S. ZechmeisterBoltenstern, "Nitrous oxide emissions from soils: how well do we understand the processes and their controls?," The Royal Society. 
[144] e. a. Groffman, Peter M., "Climate change impacts in the united states: The third national climate assessment."

[145] J. W. Doran and T. B. Parkin, Defining and Assessing Soil Quality, ch. 1, pp. 121. John Wiley Sons, Ltd, 2015.

[146] A. Paez-Garcia, C. M. Motes, W.-R. Scheible, R. Chen, E. B. Blancaflor, and M. J. Monteros, "Root traits and phenotyping strategies for plant improvement," Plants, vol. 4, no. 2, pp. 334-355, 2015.

[147] G. T. Beemster and T. I. Baskin, "Analysis of cell division and elongation underlying the developmental acceleration of root growth in arabidopsis thaliana," Plant Physiology, vol. 116, no. 4, pp. 1515-1526, 1998.

[148] E. B. Blancaflor and P. H. Masson, "Plant gravitropism. unraveling the ups and downs of a complex process," Plant Physiology, vol. 133, no. 4, pp. 1677-1690, 2003.

[149] C. S. Buer and G. K. Muday, "The transparent testa4 mutation prevents flavonoid synthesis and alters auxin transport and the response of arabidopsis roots to gravity and light," The Plant Cell, vol. 16, no. 5, pp. 1191-1205, 2004.

[150] H. N. P. Fu, Xiangdong, "Auxin promotes arabidopsis root growth by modulating gibberellin response," Nature.

[151] N. D. Miller, B. M. Parks, and E. P. Spalding, "Computer-vision analysis of seedling responses to light and gravity," The Plant Journal, vol. 52, no. 2, pp. $374-381$.

[152] C. D. Chin, "Microfluidics-based diagnostics of infectious diseases in the developing world," Nature Medicine.

[153] V. Kamat, S. Pandey, K. Paknikar, and D. Bodas, "A facile one-step method for cell lysis and dna extraction of waterborne pathogens using a microchip," Biosensors and Bioelectronics, vol. 99, pp. 62 - 69, 2018.

[154] "Microfluidic differential immunocapture biochip for specific leukocyte counting," Nature Protocols.

[155] M. Safdar, "Microfluidic fuel cells for energy generation," Lab on Chip. 
[156] D. Fuerth and A. Bazylak, "Up-Scaled Microfluidic Fuel Cells With Porous FlowThrough Electrodes," Journal of Fluids Engineering, vol. 135, 03 2013. 021102.

[157] P. N. Nge, C. I. Rogers, and A. T. Woolley, "Advances in microfluidic materials, functions, integration, and applications," Chemical Reviews, vol. 113, no. 4, pp. 2550-2583, 2013. PMID: 23410114.

[158] J. Deng, E. P. Orner, J. F. Chau, E. M. Anderson, A. L. Kadilak, R. L. Rubinstein, G. M. Bouchillon, R. A. Goodwin, D. J. Gage, and L. M. Shor, "Synergistic effects of soil microstructure and bacterial eps on drying rate in emulated soil micromodels," Soil Biology and Biochemistry, vol. 83, pp. 116 - 124, 2015.

[159] G. Grossmann, W.-J. Guo, D. W. Ehrhardt, W. B. Frommer, R. V. Sit, S. R. Quake, and M. Meier, "The rootchip: An integrated microfluidic chip for plant science," The Plant Cell, vol. 23, no. 12, pp. 4234-4240, 2011.

[160] M. Held, A. P. Lee, C. Edwards, and D. V. Nicolau, "Microfluidics structures for probing the dynamic behaviour of filamentous fungi," Microelectronic Engineering, vol. 87, no. 5, pp. $786-789,2010$. The 35th International Conference on Micro- and Nano-Engineering (MNE).

[161] D. Nichols, N. Cahoon, E. M. Trakhtenberg, L. Pham, A. Mehta, A. Belanger, T. Kanigan, K. Lewis, and S. S. Epstein, "Use of ichip for high-throughput in situ cultivation of "uncultivable" microbial species," Applied and Environmental Microbiology, vol. 76, no. 8, pp. 2445-2450, 2010.

[162] C. E. Stanley, M. Stöckli, D. van Swaay, J. Sabotič, P. T. Kallio, M. Künzler, A. J. deMello, and M. Aebi, "Probing bacterial-fungal interactions at the single cell level," Integrative Biology, vol. 6, pp. 935-945, 082014.

[163] K. Aleklett, "Build your own soil: exploring microfluidics to create microbial habitat structures," The ISME Journal.

[164] W. Busch, B. Moore, and B. Martsberger, "A microfluidic device and computational platform for high-throughput live imaging of gene expression," Nature Methods.

[165] C. Granier, "Phenopsis, an automated platform for reproducible phenotyping of plant responses to soil water deficit in arabidopsis thaliana permitted the identification of an accession with low sensitivity to soil water deficit," New Phytologist, vol. 169, no. 3, pp. 623-635, 2006. 
[166] A. Marcus and G. Frank, "Simultaneous phenotyping of leaf growth and chlorophyll fluorescence via growscreen fluoro allows detection of stress tolerance in arabidopsis thaliana and other rosette plants.," Functional Plant Biology.

[167] H. Jiang, Z. Xu, M. R. Aluru, and L. Dong, "Plant chip for high-throughput phenotyping of arabidopsis," Lab Chip, vol. 14, pp. 1281-1293, 2014.

[168] C. E. Stanley, J. Shrivastava, R. Brugman, E. Heinzelmann, D. van Swaay, and G. Grossmann, "Dual-flow-rootchip reveals local adaptations of roots towards environmental asymmetry at the physiological and genetic levels," New Phytologist, vol. 217, no. 3, pp. 1357-1369, 2018.

[169] T. Burrell, "The microphenotron: a robotic miniaturized plant phenotyping platform with diverse applications in chemical biology," Plant Methods.

[170] J. A. Aufrecht, C. M. Timm, A. Bible, J. L. Morrell-Falvey, D. A. Pelletier, M. J. Doktycz, and S. T. Retterer, "Quantifying the spatiotemporal dynamics of plant root colonization by beneficial bacteria in a microfluidic habitat," Advanced Biosystems, vol. 2, no. 6, p. 1800048, 2018.

[171] Y.-H. Park, N. Lee, G. Choi, and J.-K. Park, "Plant array chip for the germination and growth screening of arabidopsis thaliana," Lab Chip, vol. 17, pp. 3071-3077, 2017.

[172] L. Dong, "High-throughput phenotyping of morphological traits and nutrient uptake of plants using microfluidics devices."

[173] J. A. Aufrecht, J. M. Ryan, S. Hasim, and D. P. Allison, "Imaging the root hair morphology of arabidopsis seedlings in a two-layer microfluidic platform," JoVE.

[174] H. H. Chai, F. Chen, S. J. Zhang, Y. D. Li, Z. S. Lu, Y. J. Kang, and L. Yu, "Multi-chamber petaloid root-growth chip for the non-destructive study of the development and physiology of the fibrous root system of oryza sativa," Lab Chip, vol. 19, pp. 2383-2393, 2019.

[175] J. Macagno, M. R. Lescano, and C. L. Berli, "Milli-channel array for direct and quick reading of root elongation bioassays," Ecotoxicology and Environmental Safety, vol. 178, pp. 51 - 57, 2019.

[176] H. Jiang, M. A. Ali, Y. Jiao, B. Yang, and L. Dong, "In-situ, real-time monitoring of nutrient uptake on plant chip integrated with nutrient sensor," in 2017 19th 
International Conference on Solid-State Sensors, Actuators and Microsystems (TRANSDUCERS), pp. 289-292, June 2017.

[177] P. Laboratories, “Tc media-composition," in PhytoTechnology Laboratories.

[178] C. Monk, "Ecological importance of root/shoot ratios," Bulletin of the Torrey Botanical Club, 1966.

[179] M. Pérez-Patricio, J. Camas-Anzueto, A. Sanchez-Alegría, A. AguilarGonzález, F. Gutiérrez-Miceli, E. Escobar-Gómez, Y. Voisin, C. Rios-Rojas, and R. Grajales-Coutiño, "Optical method for estimating the chlorophyll contents in plant leaves," Sensors, vol. 18, p. 650, Feb 2018.

[180] R. Muñoz-Huerta, R. Guevara-Gonzalez, L. Contreras-Medina, I. Torres-Pacheco, J. Prado-Olivarez, and R. Ocampo-Velazquez, "A review of methods for sensing the nitrogen status in plants: Advantages, disadvantages and recent advances," Sensors, vol. 13, p. 10823-10843, Aug 2013.

[181] J.-K. Zhu, "Salt and drought stress signal transduction in plants," Annual Review of Plant Biology, vol. 53, no. 1, pp. 247-273, 2002. PMID: 12221975. 
VITA

\section{LAMAR BURTON}

Born, Hammond, Louisiana, USA

2012-2016

B.S., Agriculture and Animal Science

Southern University A\&M College

Baton Rouge, LA

2016-2018

M.S., Electrical and Computer Engineering Florida International University

Miami, Florida

2018-2020

Doctoral Candidate, Electrical Engineering Florida International University

Miami, Florida

\section{PUBLICATIONS AND PRESENTATIONS}

L. Burton, N. Dave, R.E. Fernandez,K. Jayachandran, S.Bhansali, "Smart gardening IoT soil sheets for real-time nutrient analysis" Journal of the Electrochemical Society, May 2018 - Inpress

L. Burton,K. Jayachandran, S. Bhansali, “The real-time revolution for in-situ soil nutrient sensing: A review," Journal of the Electrochemical Society, January 2020

L. Burton, Y. Mekonnen, A Sarwat, et al., "Exploring Wireless Sensor Network Technology in Sustainable Okra Garden: A Comparative Analysis of Okra Grown in Different Fertilizer Treatments", 14th International Society of Precision Agriculture, June 24-27 2018

Y. Mekonnen, L. Burton, A Sarwat, S. Bhansali, "IoT Sensor Network Approach for Smart Farming: An Application in Food, Energy and Water System”, 2018 IEEE GHTC, Oct. 2018

S. Namuduri, B.N.Narayanan, V.S.P. Davuluru, L. Burton, S. Bhansali, "Deep Learning Methods for Sensor based Predictive Maintenance and Future Perspectives for Electrochemical Sensors”, Journal of Electrochemical Society, January 2020

L. Burton, V. Kamat, K. Jayachandran, S. Bhansali, "SensePod for real-time evaluation of plant phenotypic characteristics," Journal of Biosensors and Bioelectronics, January 2020 - Under Review 
Y. Mekonnen, L. Burton, A. Sarwat, S. Bhansali, "Sensors, IoT, Smart Agriculture and Future of Smart City", Submitted to Journal of Electrochemical Society, 2020, (Under Review)

Y. Mekonnen, S. Namduri, L. Burton, A. Sarwat, S. Bhanslai, "Machine Learning Techniques in Wireless Sensor Network based Precision Agriculture", Journal of Electrochemical Society, January 2020 (In press) 